\title{
Late-stage $N$-Me Selective Arylation of Trialkylamines Enabled by Ni/Photoredox Dual Catalysis
}

\author{
Yangyang Shen, Tomislav Rovis* \\ Correspondence to: tr2504@columbia.edu \\ Department of Chemistry, Columbia University, New York, NY, 10027, USA.
}




\section{Table of contents}

I. General considerations S3

II. Optimization details and $\mathrm{H}_{2} \mathrm{O}$ titration $\quad \mathrm{S4}$

$\begin{array}{ll}\text { III. Deuteration studies } & \text { S7 }\end{array}$

$\begin{array}{lll}\text { IV. N-Me selective arylation } & \text { S8 }\end{array}$

$\begin{array}{lll}\text { V. References } & \text { S23 }\end{array}$

VI. ${ }^{1} \mathrm{H},{ }^{13} \mathrm{C}$ and ${ }^{19}$ F NMR spectra $\quad$ S24 


\section{General considerations}

Reagents: Unless otherwise noted, all reactions were carried out in reaction tubes with screw cap. Anhydrous solvents were purchased from Sigma Aldrich and used without further purification. All other reagents were purchased from commercial sources and used as received. For the pharmaceuticals received in salt form, a simple treatment with ethyl acetate and $\mathrm{K}_{2} \mathrm{CO}_{3}$ (aq.) or $\mathrm{KOH}(1 \mathrm{M})$ offered pure enough sample in the organic phase for the reactions. Purification of the product was conducted manually on SiliCycle ${ }^{\circledR}$ SilicaFlash ${ }^{\circledR}$ P60 (230-400 mesh) silica gel columns. Thin layer chromatography (TLC) was performed on Silicycle $250 \mu \mathrm{m}$ silica gel $60 \AA$ plates. Visualization was accomplished with UV lamp (254 nm), $\mathrm{KMnO}_{4}$ or Iodine. A Kessil blue LED (34W maximum, 24 VDC, $440 \mathrm{~nm}$ ) was used as the light source for the photoredox catalyzed reactions. The procedures described in this section are representative. Thus, the yields may differ slightly from those given in the tables of the manuscript.

Analytical Methods: ${ }^{1} \mathrm{H}$ NMR, ${ }^{13} \mathrm{C}$ NMR and ${ }^{19} \mathrm{~F}$ NMR spectra are included for all new compounds. ${ }^{1} \mathrm{H}$ NMR, ${ }^{13} \mathrm{C}$ NMR and ${ }^{19} \mathrm{~F}$ NMR spectra were recorded on Varian 300/400 MHz or Bruker 400/500 MHz at ambient temperature. All ${ }^{1} \mathrm{H}$ NMR spectra are reported in parts per million (ppm) downfield of TMS and were measured relative to the signals for $\mathrm{CHCl}_{3}(7.26 \mathrm{ppm})$. All ${ }^{13} \mathrm{C}$ NMR spectra were reported in ppm relative to residual $\mathrm{CHCl}_{3}$ (77.16 ppm) and were obtained with ${ }^{1} \mathrm{H}$ decoupling. Coupling constants, $J$, are reported in hertz $(\mathrm{Hz})$. In the case of diastereomeric mixtures, isolated NMR was recorded to determine the ratio. High resolution mass spectra (HRMS) were obtained from the Columbia University Chemistry Department Mass Spectrometry Facility on a Waters XEVO G2XS QToF mass spectrometer equipped with a UPC2 SFC inlet and a LockSpray source with one of the following three probes: electrospray ionization (ESI) probe, atmospheric pressure chemical ionization (APCI) probe, or atmospheric pressure solids analysis probe (ASAP). 


\section{Optimization details and control experiments}

\section{Table S1. Screening of Ni-catalysts}

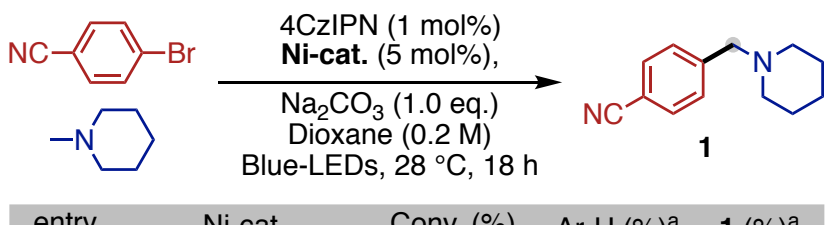

$\begin{array}{ccccc}\text { entry } & \mathrm{Ni}-\mathrm{cat} . & \text { Conv. }(\%) & \mathrm{Ar}-\mathrm{H}(\%)^{\mathrm{a}} & \mathbf{1}(\%)^{\mathrm{a}} \\ 1 & \mathrm{Ni-1} & 100 & 12 & 49 \\ 2 & \mathrm{Ni}(\mathrm{COD})_{2}{ }^{\mathrm{b}} & 100 & 27 & 5 \\ 3 & \mathrm{NiCl}_{2}\left(\mathrm{H}_{2} \mathrm{O}\right)_{6}{ }^{\mathrm{b}} & 100 & 19 & 48 \\ 4 & \mathrm{Ni}(\mathrm{OAc})_{2}\left(\mathrm{H}_{2} \mathrm{O}\right)_{4}{ }^{\mathrm{b}} & 20 & 12 & 4 \\ 5 & \mathrm{Ni}(\mathrm{acac})_{2}{ }^{\mathrm{b}} & 100 & 36 & 45 \\ 6 & \mathrm{Ni}\left(\mathrm{PPh}_{3}\right) \mathrm{Cl}_{2}^{\mathrm{b}} & 83 & 17 & 40\end{array}$

$0.2 \mathrm{mmol}$ scale, amine (2.0 equiv), mass balance acounts for homocoupling of ArBr. ${ }^{a}$ GC-MS yield with ethyl benzoate as internal standard. ${ }^{\mathrm{b}}$ dtbbpy (6 mol\%) as ligand.

\section{Table S2. Screening of Bases}

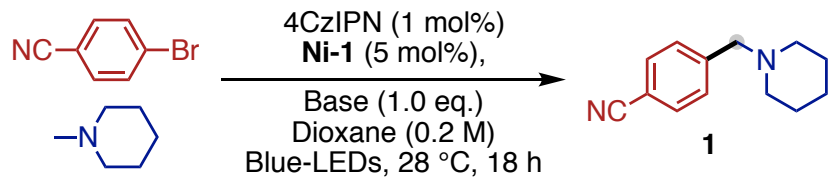

$\begin{array}{ccccc}\text { entry } & \text { Base } & \text { Conv. (\%) } & \mathrm{Ar}-\mathrm{H}(\%)^{\mathrm{a}} & \mathbf{1}(\%)^{\mathrm{a}} \\ 1 & \mathrm{Na}_{2} \mathrm{CO}_{3} & 100 & 12 & 49 \\ 2 & \mathrm{~K}_{2} \mathrm{CO}_{3} & 100 & 12 & 44 \\ 3 & \mathrm{Cs}_{2} \mathrm{CO}_{3} & 100 & 11 & 43 \\ 4 & \mathrm{NaHCO}_{3} & 83 & 7 & 40 \\ 5 & \mathrm{~K}_{2} \mathrm{HPO}_{4} & 100 & 16 & 44 \\ 6 & \mathrm{~K}_{3} \mathrm{PO}_{4} & 100 & 12 & 46 \\ 7 & \mathrm{DABCO} & 48 & 11 & 15 \\ 8 & 2,4,6-C o l l i d i n e & 100 & 4 & 43 \\ 9 & \mathrm{Barton's} \mathrm{base} & 100 & 10 & 33 \\ 10 & \mathrm{LiOPiv}_{10} & 100 & 39 & 47 \\ 11 & \mathrm{NaOPiv}_{12} & 100 & 94 & 5 \\ 13 & \mathrm{CsOPiv}_{10} & 100 & 96 & 4 \\ 14 & \mathrm{NaO}_{2} \mathrm{CAd} & 100 & 86 & 11 \\ 15 & \mathrm{KO}_{2} \mathrm{CAd} & 100 & 91 & 6 \\ & \mathrm{CsO}_{2} \mathrm{CAd} & 100 & 83 & 8\end{array}$

$0.2 \mathrm{mmol}$ scale, amine (2.0 equiv), mass balance acounts for homocoupling of $\mathrm{ArBr} .{ }^{a} \mathrm{GC}-\mathrm{MS}$ yield with ethyl benzoate as internal standard. 
Table S3. $\mathrm{H}_{2} \mathrm{O}$ Titration

\begin{tabular}{|c|c|c|c|c|c|}
\hline $\mathrm{NC}-$ & $\sqrt{-B r}$ & $\begin{array}{r}4 \mathrm{Czlf} \\
\mathrm{Ni}-1 \\
\mathrm{Na}_{2} \mathrm{C} \\
\mathrm{Diox} \\
\text { Blue-LE }\end{array}$ & $\begin{array}{l}(1 \mathrm{~mol} \%) \\
\mathrm{mol} \%), \\
\underset{(1.0 \mathrm{eq} .)}{\longrightarrow} \\
(0.2 \mathrm{M}) \\
28^{\circ} \mathrm{C}, 18 \mathrm{~h}\end{array}$ & 1 & \\
\hline entry & $\mathrm{H}_{2} \mathrm{O}$ (x eq.) & ) as add. & Conv. (\%) & $\mathrm{Ar}-\mathrm{H}(\%)^{\mathrm{a}}$ & $1(\%)^{a}$ \\
\hline 1 & 0 & & 100 & 12 & 49 \\
\hline 2 & 5 & & 100 & 16 & 38 \\
\hline 3 & 10 & & 100 & 31 & 38 \\
\hline 4 & 20 & & 100 & 59 & 29 \\
\hline 5 & 50 & & 100 & 90 & 3 \\
\hline 6 & 100 & & 55 & 54 & 0 \\
\hline 7 & 200 & & 81 & 77 & 0 \\
\hline
\end{tabular}

$0.2 \mathrm{mmol}$ scale, amine (2.0 equiv), mass balance accounts for homocoupling of $\mathrm{ArBr} .{ }^{\mathrm{a}} \mathrm{GC}-\mathrm{MS}$ yield with ethyl benzoate as internal standard.

Table S4. Conditions for Hindered Amine Substrate

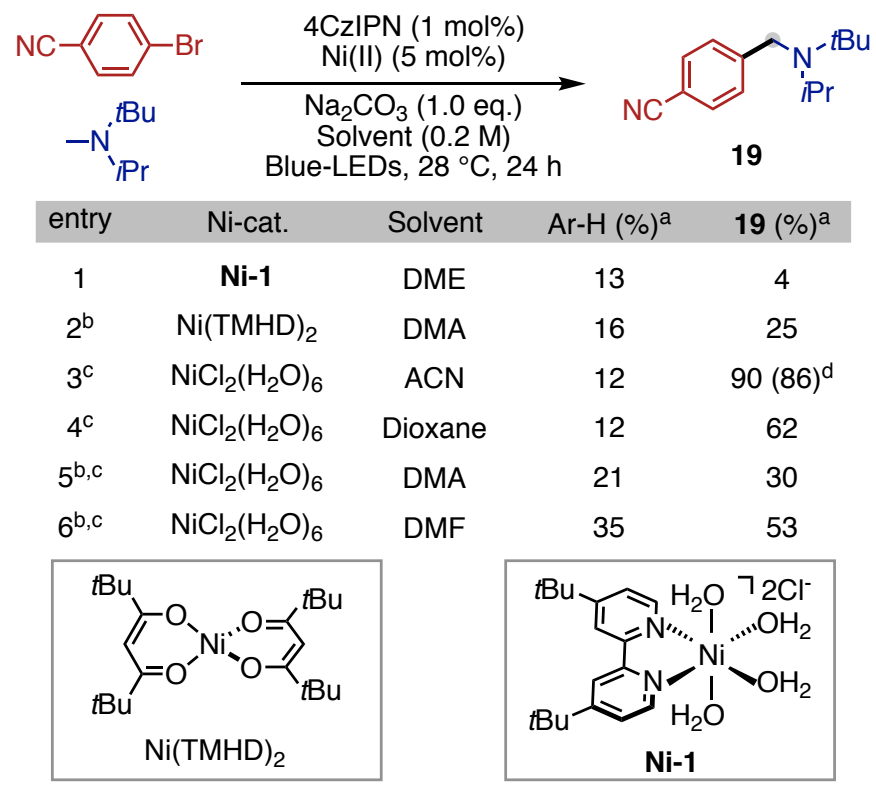

$0.2 \mathrm{mmol}$ scale, amine (2.0 equiv), mass balance acounts for homocoupling of $\mathrm{ArBr}$, full conversion. ${ }^{a} \mathrm{GC}-\mathrm{MS}$ yield with ethyl benzoate as inter standard. ${ }^{b}$ Dimerization of amine was detected. ${ }^{c} 10 \mathrm{~mol} \%$ of dipivaloyl methane was used as ligand.

$\mathrm{d}$ isolated yield. 
Table S5. Ratio and Concentration of Starting Materials

\begin{tabular}{|c|c|c|c|c|}
\hline $\mathrm{NC}-\mathrm{N}$ & Blue & $\begin{array}{l}\text { IPN }(1 \mathrm{~mol} \\
-1(5 \mathrm{~mol} \% \\
\mathrm{CO}_{3}(1.0 \mathrm{e} \\
E D s, 28^{\circ} \mathrm{C}\end{array}$ & $\overrightarrow{8 \mathrm{~h}}$ & 1 \\
\hline entry & amine: $\mathrm{ArBr}$ & Dioxane & $\operatorname{Ar}-\mathrm{H}(\%)^{\mathrm{a}}$ & $1(\%)^{a}$ \\
\hline 1 & $2: 1$ & $0.2 \mathrm{M}$ & 12 & $49(50)^{\mathrm{b}}$ \\
\hline 2 & $1: 1$ & $0.2 \mathrm{M}$ & 23 & 33 \\
\hline 3 & $2: 1$ & $0.1 \mathrm{M}$ & 25 & 46 \\
\hline 4 & $2: 1$ & $0.067 \mathrm{M}$ & 28 & 44 \\
\hline $5^{c}$ & 2: 1 & $0.05 \mathrm{M}$ & 29 & 43 \\
\hline
\end{tabular}

$0.2 \mathrm{mmol}$ scale, amine (2.0 equiv), full conversion of $\mathrm{ArBr}$, mass balance acounts for homocoupling of $\mathrm{ArBr}$. a GC-MS yield with ethyl benzoate as inter standard. ${ }^{b}$ isolated yield. ${ }^{c}$ $87 \%$ conversion.

Table S6. Photocatalysts Screening
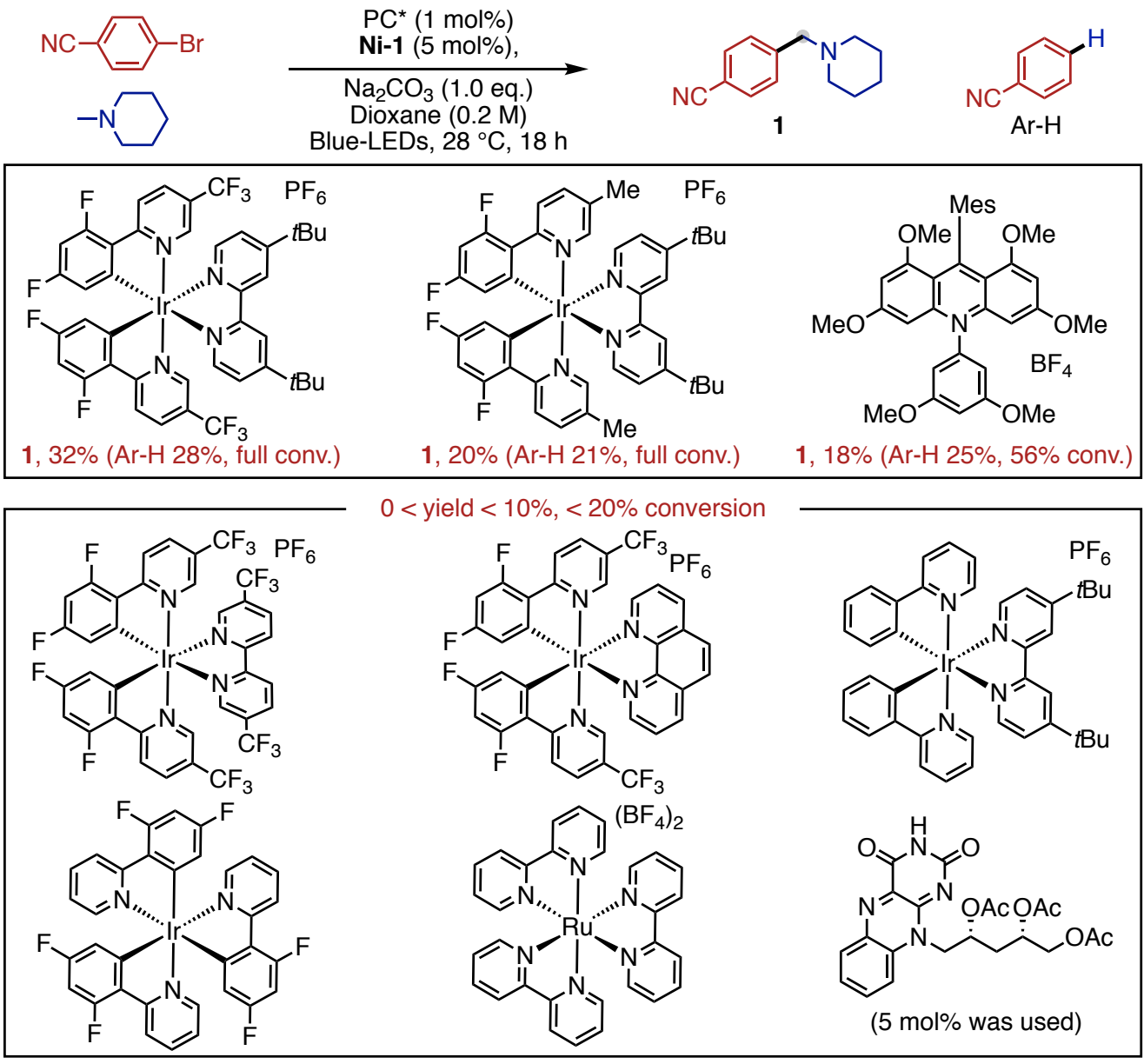

$0.2 \mathrm{mmol}$ scale, amine (2.0 equiv), mass balance acounts for homocoupling of $\mathrm{ArBr} .{ }^{\mathrm{a}} \mathrm{GC}-\mathrm{MS}$ yield with ethyl benzoate as inter standard. 


\section{Deuteration studies}

$\alpha$ - and $\beta$ - deuterated tributylamines were synthesized according to reported literatures. ${ }^{1-3}$
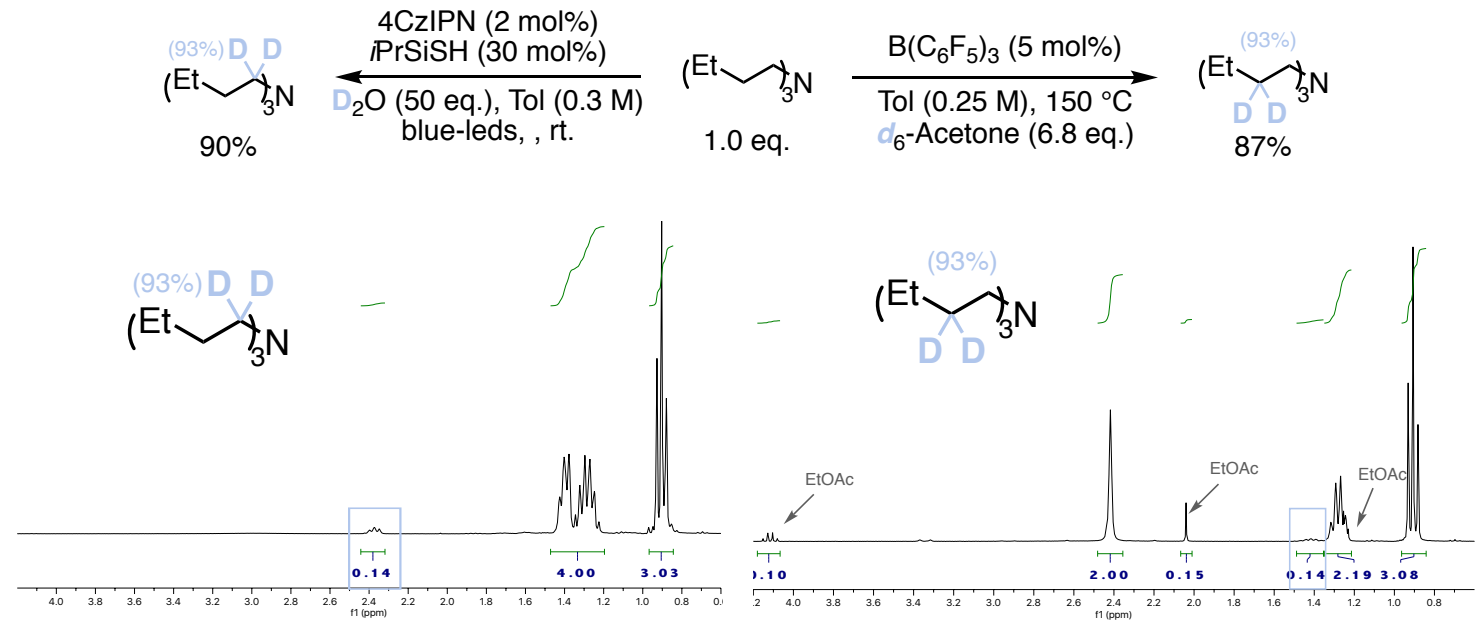

Figure S1. Synthesis of deuterated tributylamine

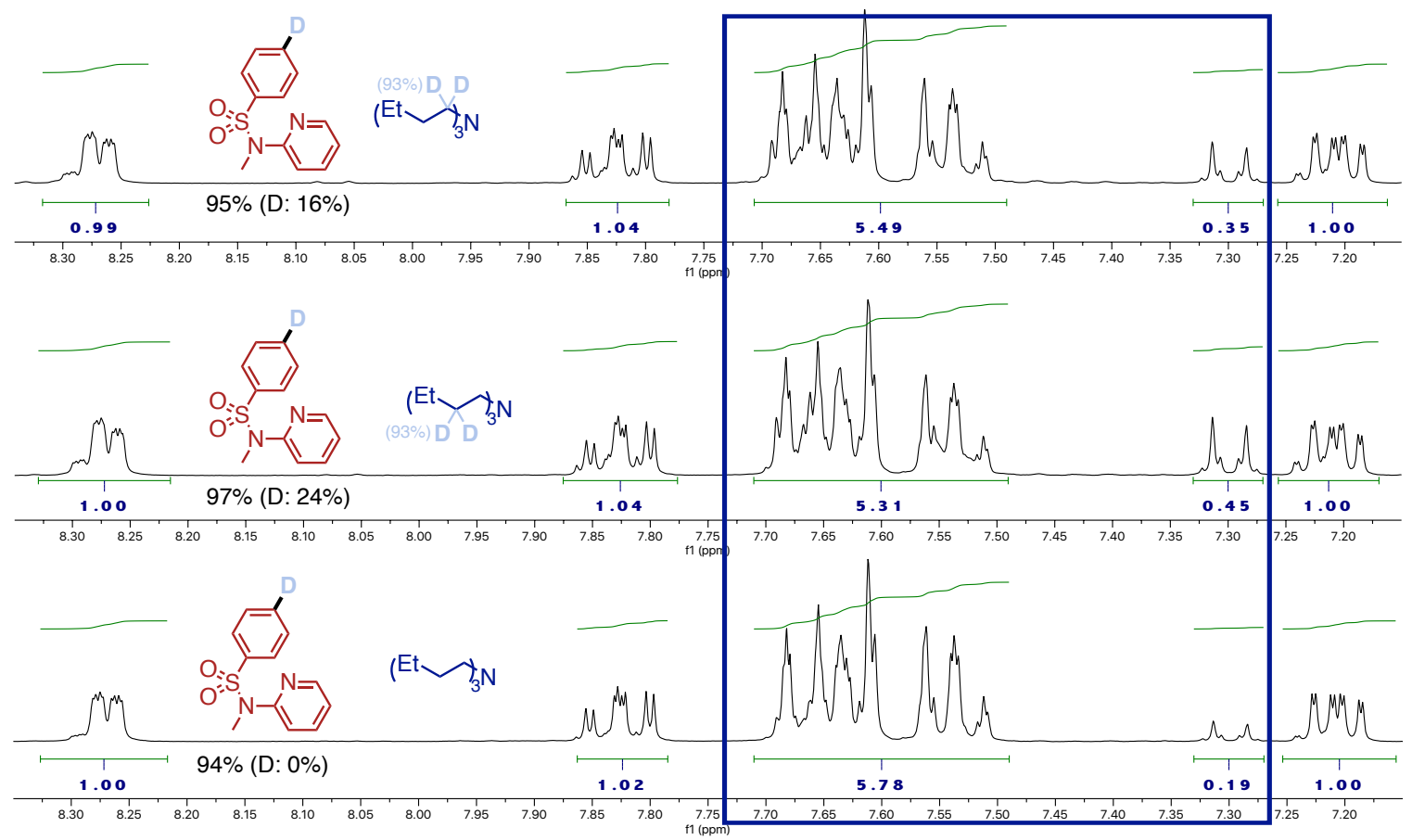

Figure S2. ${ }^{1} \mathrm{H}-\mathrm{NMR}$ of hydrodebromination with tributylamine 


\section{IV. $N$-Me selective arylation}

General Procedure A for the $\mathrm{N}$-Me arylation of simple trialkylamines. A $8.0 \mathrm{~mL}$ disposable borosilicate glass tube with screw cap containing a stir bar was charged with 4CzIPN (1 mol\%, $1.6 \mathrm{mg}, 0.002 \mathrm{mmol}), \mathrm{NiCl}_{2}(\mathrm{dtbbpy})\left(\mathrm{H}_{2} \mathrm{O}\right)_{4}(5 \mathrm{~mol} \%, 4.7 \mathrm{mg}, 0.01$ mmol), $\mathrm{Na}_{2} \mathrm{CO}_{3}(21.2 \mathrm{mg}, 0.2 \mathrm{mmol})$, trialkylamine $(0.4 \mathrm{mmol}$, added after solvent if volatile) and aryl bromide (0.2 mmol, added after solvent if liquid). The tube was transferred to a nitrogen-filled glove-box where the dry 1,4-Dioxane $(1.0 \mathrm{~mL}, 0.2 \mathrm{M})$ was added. Then the reaction was stirred for 1 minute and transferred outside, placing $\sim 10 \mathrm{~cm}$ away from a Kessil blue LED (34W maximum, $24 \mathrm{VDC}, 440 \mathrm{~nm}$ ) and vigorously stirred for $18 \mathrm{~h}$ with cooling by fan. After completion of the reaction, the mixture was filter through a short pad of celite and purified by flash column chromatography on silica gel with EtOAc/Hexane.

Note: for the arylation of complex trialkylamines, the reaction scale is $0.1 \mathrm{mmol}$ in Dioxane (0.1 M) for $24 \mathrm{~h}$.

General Procedure B for the $\mathrm{N}$-Me arylation of sterically hindered trialkylamines. A $8.0 \mathrm{~mL}$ disposable borosilicate glass tube with screw cap containing a stir bar was charged with 4CzIPN (1 mol\%, $1.6 \mathrm{mg}, 0.002 \mathrm{mmol}), \mathrm{NiCl}_{2}\left(\mathrm{H}_{2} \mathrm{O}\right)_{6}(5 \mathrm{~mol} \%, 2.4 \mathrm{mg}, 0.01 \mathrm{mmol})$, $\mathrm{Na}_{2} \mathrm{CO}_{3}(21.2 \mathrm{mg}, 0.2 \mathrm{mmol})$ and aryl bromide $(0.2 \mathrm{mmol}$, added after solvent if liquid). The tube was transferred to a nitrogen-filled glove-box where the dry 1,4-Dioxane $(1.0 \mathrm{~mL}$, $0.2 \mathrm{M}), N$-isopropyl- $N$-methyl-tert-butylamine $(0.4 \mathrm{mmol}, 67.4 \mu \mathrm{L})$ and dipivaloylmethane (10 mol\%, $4.2 \mu \mathrm{L}, 0.02 \mathrm{mmol}$ ) were added. Then the reaction was stirred for 1 minute and transferred outside, placing $\sim 10 \mathrm{~cm}$ away from a Kessil blue LED (34W maximum, 24 VDC, $440 \mathrm{~nm}$ ) and vigorously stirred for $18 \mathrm{~h}$ with cooling by fan. After completion of the reaction, the mixture was filter through a short pad of celite and purified by flash column chromatography on silica gel with EtOAc/Hexane.

Both ${ }^{1} H-N M R$ and ${ }^{13} C-N M R$ of Products $1^{6}, 3^{11 e}$ and $4^{6}$ are in good consistency with reported data in literatures.<smiles>CCN(C)Cc1ccc(C#N)cc1</smiles>

4-((ethyl(methyl)amino)methyl)benzonitrile (2). Following the general procedure A, the 
title compound was obtained in $72 \%$ yield $(25.1 \mathrm{mg})$ as colorless oil. ${ }^{1} \mathrm{H}$ NMR $(400 \mathrm{MHz}$, $\left.\mathrm{CDCl}_{3}\right) \delta 7.60(\mathrm{~d}, J=8.3 \mathrm{~Hz}, 2 \mathrm{H}), 7.44(\mathrm{~d}, J=8.3 \mathrm{~Hz}, 2 \mathrm{H}), 3.52$ (s, 2H), 2.45 (q, $J=7.1$ $\mathrm{Hz}, 2 \mathrm{H}), 2.18$ (s, 3H), 1.09 (t, $\left.J=7.1 \mathrm{~Hz}, 3 \mathrm{H}) .{ }^{13} \mathrm{C} \mathrm{NMR} \mathrm{(101} \mathrm{MHz,} \mathrm{CDCl}_{3}\right) \delta 145.46$, 132.21, 129.57, 119.15, 110.87, 61.66, 51.56, 41.87, 12.58. HRMS calcd. for $\mathrm{C}_{11} \mathrm{H}_{14} \mathrm{~N}_{2}$ $[\mathrm{M}+\mathrm{H}]^{+}:$175.1235, found 175.1246 .

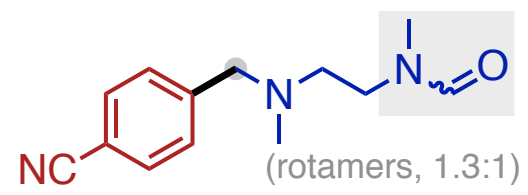

$N$-(2-((4-cyanobenzyl)(methyl)amino)ethyl)- $N$-methylformamide (5). Following the general procedure A, the title compound was obtained in $78 \%$ yield $(36.1 \mathrm{mg})$ as colorless oil. ${ }^{1} \mathrm{H}$ NMR $\left(400 \mathrm{MHz} \mathrm{CDCl}_{3}\right) \delta[8.01(\mathrm{~s})+8.00(\mathrm{~s}), 1 \mathrm{H}], 7.58-7.55(\mathrm{~m}, 2 \mathrm{H}), 7.41-7.36$ $(\mathrm{m}, 2 \mathrm{H}),[3.56(\mathrm{~s})+3.55(\mathrm{~s}), 2 \mathrm{H}],[3.46(\mathrm{t}, J=6.5 \mathrm{~Hz}, 0.88 \mathrm{H})+3.29(\mathrm{t}, J=6.0 \mathrm{~Hz}, 1.12 \mathrm{H})]$, $[2.92(\mathrm{~s}, 1.3 \mathrm{H})+2.74(\mathrm{~s}, 1.7 \mathrm{H})],[2.55(\mathrm{t}, J=6.5 \mathrm{~Hz}, 0.9 \mathrm{H})+2.49(\mathrm{t}, J=6.0 \mathrm{~Hz}, 1.1 \mathrm{H})]$, $[2.22(\mathrm{~s})+2.21(\mathrm{~s}), 3 \mathrm{H}] .{ }^{13} \mathrm{C} \mathrm{NMR}\left(101 \mathrm{MHz}, \mathrm{CDCl}_{3}\right) \delta(163.03,162.67),(144.85,144.61)$, (132.26, 132.13), (129.42, 129.31), (118.96, 118.87), (111.08, 110.91), (62.30, 61.77), (54.75, 54.21), (47.54, 42.32), (41.91, 35.00), (29.86, 29.71). HRMS calcd. for $\mathrm{C}_{13} \mathrm{H}_{17} \mathrm{~N}_{3} \mathrm{O}$ $[\mathrm{M}+\mathrm{H}]^{+}:$232.1450, found 232.1462.

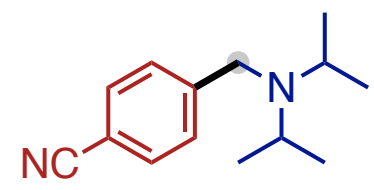

4-((diisopropylamino)methyl)benzonitrile (6). Following the general procedure $A$, the title compound was obtained in $77 \%$ yield $(33.3 \mathrm{mg})$ as colorless oil. ${ }^{1} \mathrm{H}$ NMR (400 MHz, $\left.\mathrm{CDCl}_{3}\right) \delta 7.59(\mathrm{~d}, J=8.3 \mathrm{~Hz}, 2 \mathrm{H}), 7.52(\mathrm{~d}, J=8.1 \mathrm{~Hz}, 2 \mathrm{H}), 3.71(\mathrm{~s}, 2 \mathrm{H}), 3.02(\mathrm{~m}, 2 \mathrm{H})$, $1.04(\mathrm{~d}, J=6.6 \mathrm{~Hz}, 12 \mathrm{H}) .{ }^{13} \mathrm{C} \mathrm{NMR}\left(101 \mathrm{MHz}, \mathrm{CDCl}_{3}\right) \delta 149.78,132.03,128.52,119.43$, 110.07, 49.14, 48.59, 20.89. HRMS calcd. for $\mathrm{C}_{14} \mathrm{H}_{20} \mathrm{~N}_{2}[\mathrm{M}+\mathrm{H}]^{+}:$217.1705, found 217.1717.<smiles>N#Cc1ccc(CN(C2CCCCC2)C2CCCCC2)cc1</smiles> 
4-((dicyclohexylamino)methyl)benzonitrile (7). Following the general procedure $\mathrm{A}$, the title compound was obtained in 75\% yield (44.5 mg) as colorless oil. ${ }^{1} \mathrm{H}$ NMR (400 MHz, $\left.\mathrm{CDCl}_{3}\right) \delta 7.55(\mathrm{~d}, J=8.3 \mathrm{~Hz}, 2 \mathrm{H}), 7.48(\mathrm{~d}, J=8.3 \mathrm{~Hz}, 2 \mathrm{H}), 3.78(\mathrm{~s}, 2 \mathrm{H}), 2.53-2.47(\mathrm{~m}$, $2 \mathrm{H}), 1.75-1.55(\mathrm{~m}, 10 \mathrm{H}), 1.27-0.97(\mathrm{~m}, 10 \mathrm{H}) .{ }^{13} \mathrm{C} \mathrm{NMR}\left(101 \mathrm{MHz}, \mathrm{CDCl}_{3}\right) \delta 150.23$, 131.99, 128.41, 119.51, 109.93, 58.32, 50.22, 32.07, 26.49, 26.32. HRMS calcd. for $\mathrm{C}_{20} \mathrm{H}_{28} \mathrm{~N}_{2}[\mathrm{M}+\mathrm{H}]^{+}:$297.2331, found 297.2343.<smiles>CCOC(=O)C1CCCCN1Cc1ccc(C#N)cc1</smiles>

ethyl 1-(4-cyanobenzyl)piperidine-2-carboxylate (8). Following the general procedure A, the title compound was obtained in $51 \%$ yield $(27.8 \mathrm{mg})$ as colorless oil. ${ }^{1} \mathrm{H}$ NMR (400 $\left.\mathrm{MHz} \mathrm{CDCl}_{3}\right) \delta 7.58(\mathrm{~d}, J=8.2 \mathrm{~Hz}, 2 \mathrm{H}), 7.47(\mathrm{~d}, J=8.1 \mathrm{~Hz}, 2 \mathrm{H}), 4.4-4.12(\mathrm{~m}, 2 \mathrm{H}), 3.83$ $(\mathrm{d}, J=14.2 \mathrm{~Hz}, 1 \mathrm{H}), 3.44(\mathrm{~d}, J=14.2 \mathrm{~Hz}, 1 \mathrm{H}), 3.18(\mathrm{dd}, J=7.1,4.6 \mathrm{~Hz}, 1 \mathrm{H}), 2.87$ (dt, $J$ $=10.5,4.6 \mathrm{~Hz}, 1 \mathrm{H}), 2.19-2.13(\mathrm{~m}, 1 \mathrm{H}), 1.89-1.79(\mathrm{~m}, 2 \mathrm{H}), 1.63-1.51(\mathrm{~m}, 3 \mathrm{H}), 1.45-1.37$ $(\mathrm{m}, 1 \mathrm{H}), 1.27(\mathrm{t}, J=7.1 \mathrm{~Hz}, 3 \mathrm{H}) .{ }^{13} \mathrm{C} \mathrm{NMR}\left(101 \mathrm{MHz}, \mathrm{CDCl}_{3}\right) \delta 173.72,144.90,132.14$, $129.52,119.15,110.88,64.45,60.52,60.23,50.25,29.65,25.41,22.38,14.43$. HRMS calcd. for $\mathrm{C}_{16} \mathrm{H}_{20} \mathrm{~N}_{2} \mathrm{O}_{2}[\mathrm{M}+\mathrm{H}]^{+}:$273.1603, found 273.1615.

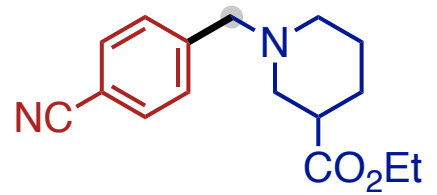

ethyl 1-(4-cyanobenzyl)piperidine-3-carboxylate (9). Following the general procedure A, the title compound was obtained in $55 \%$ yield $(30.0 \mathrm{mg})$ as colorless oil. ${ }^{1} \mathrm{H}$ NMR (400 $\left.\mathrm{MHz}_{\mathrm{CDCl}}\right) \delta 7.59(\mathrm{~d}, J=8.3 \mathrm{~Hz}, 2 \mathrm{H}), 7.43(\mathrm{~d}, J=8.2 \mathrm{~Hz}, 2 \mathrm{H}), 4.10$ (qd, $J=7.1,2.2$ $\mathrm{Hz}, 2 \mathrm{H}), 3.58-3.49(\mathrm{~m}, 2 \mathrm{H}), 2.88-2.83(\mathrm{~m}, 1 \mathrm{H}), 2.66-2.52(\mathrm{~m}, 2 \mathrm{H}), 2.27$ (t, $J=10.4 \mathrm{~Hz}$, 1H), 2.11-2.06 (m, 1H), 1.93-1.89 (m, 1H), 1.76-1.70 (m, 1H), 1.61-1.45 (m, 2H), $1.22(\mathrm{t}$, $J=7.1 \mathrm{~Hz}, 3 \mathrm{H}) .{ }^{13} \mathrm{C} \mathrm{NMR}\left(101 \mathrm{MHz}, \mathrm{CDCl}_{3}\right) \delta 174.12,144.63,132.18,129.44,119.11$, $110.90,62.79,60.44,55.56,53.91,41.93,26.85,24.60,14.32$. HRMS calcd. for $\mathrm{C}_{16} \mathrm{H}_{20} \mathrm{~N}_{2} \mathrm{O}_{2}[\mathrm{M}+\mathrm{H}]^{+}:$273.1603, found 273.1614. 


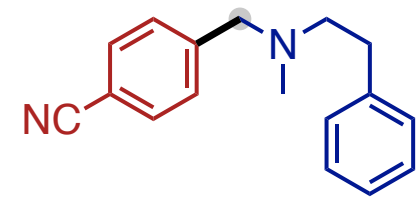

4-((methyl(phenethyl)amino)methyl)benzonitrile (10). Following the general procedure A, the title compound was obtained in $88 \%$ yield $(44.1 \mathrm{mg})$ as colorless oil. ${ }^{1} \mathrm{H}$ NMR (400 $\left.\mathrm{MHz} \mathrm{CDCl}_{3}\right) \delta$ 7.57-7.55 (m, 2H), 7.37-7.35 (m, 2H), 7.30-7.25 (m, 2H), 7.22-7.15 (m, $3 \mathrm{H}), 3.58(\mathrm{~s}, 2 \mathrm{H}), 2.81(\mathrm{dd}, J=8.8,6.5 \mathrm{~Hz}, 2 \mathrm{H}), 2.64(\mathrm{dd}, J=8.8,6.3 \mathrm{~Hz}, 2 \mathrm{H}), 2.28$ (s, $3 \mathrm{H}) .{ }^{13} \mathrm{C} \mathrm{NMR}\left(101 \mathrm{MHz}, \mathrm{CDCl}_{3}\right) \delta 145.25,140.35,132.18,129.41,128.86,128.47$, $126.18,119.14,110.83,61.92,59.28,42.35,34.06$. HRMS calcd. for $\mathrm{C}_{17} \mathrm{H}_{18} \mathrm{~N}_{2}[\mathrm{M}+\mathrm{H}]^{+}$: 251.1548, found 251.1561.

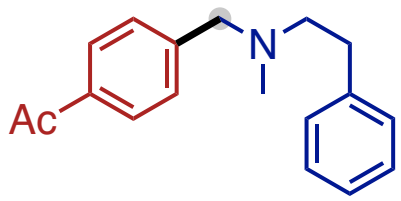

1-(4-((methyl(phenethyl)amino)methyl)phenyl)ethan-1-one (11). Following the general procedure $\mathrm{A}$, the title compound was obtained in $68 \%$ yield $(36.4 \mathrm{mg})$ as brown oil. ${ }^{1} \mathrm{H}$ NMR (400 MHz, $\left.\mathrm{CDCl}_{3}\right) \delta 7.90(\mathrm{~d}, J=8.3 \mathrm{~Hz}, 2 \mathrm{H}), 7.38(\mathrm{~d}, J=8.2 \mathrm{~Hz}, 2 \mathrm{H}), 7.30-7.26$ $(\mathrm{m}, 2 \mathrm{H}), 7.22-7.17(\mathrm{~m}, 3 \mathrm{H}), 3.61(\mathrm{~s}, 2 \mathrm{H}), 2.83(\mathrm{dd}, J=9.3,6.3 \mathrm{~Hz}, 2 \mathrm{H}), 2.67$ (dd, $J=9.2$, $6.3 \mathrm{~Hz}, 2 \mathrm{H}), 2.60(\mathrm{~s}, 3 \mathrm{H}), 2.30$ (s, 3H). ${ }^{13} \mathrm{C} \mathrm{NMR}\left(101 \mathrm{MHz}, \mathrm{CDCl}_{3}\right) \delta 197.99,145.06$, $140.44,136.15,129.01,128.84,128.46,126.12$, 61.97, 59.32, 42.37, 34.03, 26.71. HRMS calcd. for $\mathrm{C}_{18} \mathrm{H}_{21} \mathrm{NO}[\mathrm{M}+\mathrm{H}]^{+}: 268.1701$, found 268.1718 .

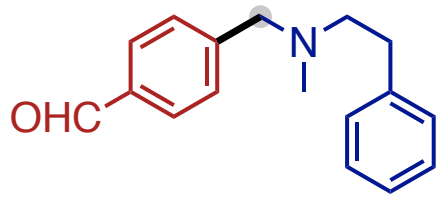

4-((methyl(phenethyl)amino)methyl)benzaldehyde (12). Following the general procedure $\mathrm{A}$, the title compound was obtained in $70 \%$ yield $(35.5 \mathrm{mg})$ as brown oil. ${ }^{1} \mathrm{H}$ NMR (400 MHz, $\left.\mathrm{CDCl}_{3}\right) \delta 9.99(\mathrm{~s}, 1 \mathrm{H}), 7.81(\mathrm{~d}, J=8.2 \mathrm{~Hz}, 2 \mathrm{H}), 7.45(\mathrm{~d}, J=8.0 \mathrm{~Hz}, 2 \mathrm{H})$, 7.30-7.26 (m, 2H), 7.22-7.17 (m, 3H), 3.63 (s, 2H), 2.83 (dd, $J=9.3,6.1 \mathrm{~Hz}, 2 \mathrm{H}), 2.67$ (dd, $J=9.0,6.2 \mathrm{~Hz}, 2 \mathrm{H}), 2.30$ (s, 3H). ${ }^{13} \mathrm{C} \mathrm{NMR}\left(101 \mathrm{MHz}, \mathrm{CDCl}_{3}\right) \delta 192.16,146.86,140.46$, 135.60, 129.91, 129.42, 128.88, 128.49, 126.18, 62.12, 59.38, 42.43, 34.10. HRMS calcd. for $\mathrm{C}_{17} \mathrm{H}_{19} \mathrm{NO}[\mathrm{M}+\mathrm{H}]^{+}: 254.1545$, found 254.1544 . 


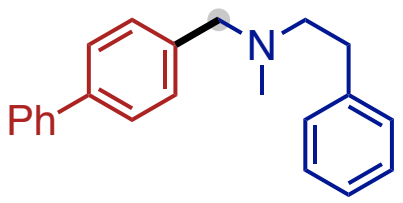

$N$-([1,1'-biphenyl]-4-ylmethyl)- $N$-methyl-2-phenylethan-1-amine (13). Following the general procedure $\mathrm{A}$, the title compound was obtained in $66 \%$ yield $(39.8 \mathrm{mg})$ as colorless oil. ${ }^{1} \mathrm{H}$ NMR (400 MHz, $\left.\mathrm{CDCl}_{3}\right) \delta$ 7.60-7.52 (m, 4H), 7.44-7.41 (m, 2H), 7.37-7.24 (m, 5H), 7.20-7.17 (m, 3H), 3.61 (s, 2H), 2.85 (dd, $J=9.5,6.2 \mathrm{~Hz}, 2 \mathrm{H}), 2.69$ (dd, $J=9.5,6.1$ $\mathrm{Hz}, 2 \mathrm{H}), 2.32$ (s, 3H). ${ }^{13} \mathrm{C}$ NMR (101 MHz, $\left.\mathrm{CDCl}_{3}\right) \delta 141.16,140.61,140.05,138.11$, 129.57, 128.91, 128.87, 128.47, 127.28, 127.19, 127.11, 126.10, 61.94, 59.28, 42.31, 34.02. HRMS calcd. for $\mathrm{C}_{22} \mathrm{H}_{23} \mathrm{~N}[\mathrm{M}+\mathrm{H}]^{+}: 302.1909$, found 302.1921 .<smiles>COc1cc(CN(C)CCc2ccccc2)cc(OC)c1</smiles>

$N$-(3,5-dimethoxybenzyl)- $N$-methyl-2-phenylethan-1-amine (14). Following the general procedure $\mathrm{A}$, the title compound was obtained in $71 \%$ yield $(40.5 \mathrm{mg})$ as brown oil. ${ }^{1} \mathrm{H}$ NMR (400 MHz, $\left.\mathrm{CDCl}_{3}\right) \delta$ 7.28-7.25 (m, 2H), 7.19-7.16 (m, 3H), 6.47 (d, $J=2.3$ $\mathrm{Hz}, 2 \mathrm{H}), 6.35$ (t, $J=2.3 \mathrm{~Hz}, 1 \mathrm{H}), 3.76(\mathrm{~s}, 6 \mathrm{H}), 3.49$ (s, 2H), 2.82 (dd, $J=9.2,6.3 \mathrm{~Hz}, 2 \mathrm{H})$, $2.65(\mathrm{dd}, J=9.3,6.3 \mathrm{~Hz}, 2 \mathrm{H}), 2.29$ (s, 3H). ${ }^{13} \mathrm{C} \mathrm{NMR}\left(101 \mathrm{MHz}, \mathrm{CDCl}_{3}\right) \delta 160.85,141.90$, $140.70,128.90,128.42,126.04,106.74,99.31,62.53,59.21,55.43,42.46,34.02$. HRMS calcd. for $\mathrm{C}_{18} \mathrm{H}_{23} \mathrm{NO}_{2}[\mathrm{M}+\mathrm{H}]^{+}:$286.1807, found 286.1820 .<smiles>CN(CCc1ccccc1)Cc1ccc(S(=O)(=O)N(C)c2ccccn2)cc1</smiles>

$N$-methyl-4-((methyl(phenethyl)amino)methyl)- $N$-(pyridin-2-yl)benzenesulfonamide (15). Following the general procedure $A$, the title compound was obtained in $53 \%$ yield (41.9 mg) as colorless oil. ${ }^{1} \mathrm{H}$ NMR (400 MHz, $\left.\mathrm{CDCl}_{3}\right) \delta 8.27(\mathrm{dt}, J=4.8,1.5 \mathrm{~Hz}, 1 \mathrm{H})$, 7.70-7.69 (m, 2H), 7.51-7.49 (m, 2H), 7.33-7.08 (m, 8H), $3.56(\mathrm{~s}, 2 \mathrm{H}), 3.28(\mathrm{~s}, 3 \mathrm{H}), 2.79$ $(\mathrm{dd}, J=8.9,6.4 \mathrm{~Hz}, 2 \mathrm{H}), 2.63(\mathrm{dd}, J=9.0,6.3 \mathrm{~Hz}, 2 \mathrm{H}), 2.26(\mathrm{~s}, 3 \mathrm{H}) .{ }^{13} \mathrm{C} \mathrm{NMR}(101 \mathrm{MHz}$, $\left.\mathrm{CDCl}_{3}\right) \delta 153.84,148.01,145.26,140.35,137.62,135.75,129.14,128.83,128.44,127.59$, 
126.13, 121.12, 120.75, 61.73, 59.28, 42.36, 35.62, 34.03. HRMS calcd. for $\mathrm{C}_{22} \mathrm{H}_{25} \mathrm{~N}_{3} \mathrm{O}_{2} \mathrm{~S}$ $[\mathrm{M}+\mathrm{H}]^{+}: 396.1746$, found 396.1785 .

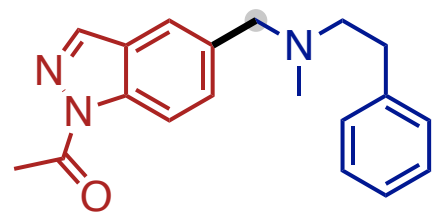

1-(5-((methyl(phenethyl)amino)methyl)-1H-indazol-1-yl)ethan-1-one (16). Following the general procedure A, the title compound was obtained in $57 \%$ yield $(35.0 \mathrm{mg})$ as yellow oil. ${ }^{1} \mathrm{H}$ NMR (400 MHz, $\left.\mathrm{CDCl}_{3}\right) \delta 8.35(\mathrm{~d}, J=8.5 \mathrm{~Hz}, 1 \mathrm{H}), 8.05(\mathrm{~s}, 1 \mathrm{H}), 7.58(\mathrm{~s}, 1 \mathrm{H}), 7.50$ (dd, $J=8.6,1.5 \mathrm{~Hz}, 1 \mathrm{H}), 7.29-7.26(\mathrm{~m}, 2 \mathrm{H}), 7.22-7.16(\mathrm{~m}, 3 \mathrm{H}), 3.67$ (s, 2H), 2.83 (dd, $J=$ 9.1, $6.4 \mathrm{~Hz}, 2 \mathrm{H}), 2.78$ (s, 3H), 2.67 (dd, $J=9.1,6.3 \mathrm{~Hz}, 2 \mathrm{H}), 2.31$ (s, 3H). ${ }^{13} \mathrm{C}$ NMR $(101$ $\left.\mathrm{MHz}, \mathrm{CDCl}_{3}\right) \delta 171.00,140.44,139.75,138.41,135.82,130.77,128.78,128.34,126.52$, 126.00, 120.62, 115.21, 61.95, 59.06, 42.15, 33.94, 23.00. HRMS calcd. for $\mathrm{C}_{19} \mathrm{H}_{21} \mathrm{~N}_{3} \mathrm{O}$ $[\mathrm{M}+\mathrm{H}]^{+}:$308.1763, found 308.1777 .<smiles>CC(=O)n1nc(CN(C)CCc2ccccc2)c2ccccc21</smiles>

1-(3-((methyl(phenethyl)amino)methyl)-1H-indazol-1-yl)ethan-1-one (17). Following the general procedure A, the title compound was obtained in 53\% yield $(32.6 \mathrm{mg}$ ) as yellow oil. ${ }^{1} \mathrm{H}$ NMR (400 MHz, $\left.\mathrm{CDCl}_{3}\right) \delta 8.40(\mathrm{~d}, J=8.4 \mathrm{~Hz}, 1 \mathrm{H}), 7.72(\mathrm{~d}, J=8.0 \mathrm{~Hz}, 1 \mathrm{H}), 7.51$ (ddd, $J=8.3,7.1,1.1 \mathrm{~Hz}, 1 \mathrm{H}), 7.29-7.24(\mathrm{~m}, 3 \mathrm{H}), 7.21-7.17(\mathrm{~m}, 3 \mathrm{H}), 3.92(\mathrm{~s}, 2 \mathrm{H}), 2.89$ $(\mathrm{dd}, J=8.9,5.8 \mathrm{~Hz}, 2 \mathrm{H}), 2.80-2.76(\mathrm{~m}, 5 \mathrm{H}), 2.36(\mathrm{~s}, 3 \mathrm{H}) .{ }^{13} \mathrm{C} \mathrm{NMR}\left(101 \mathrm{MHz}, \mathrm{CDCl}_{3}\right) \delta$ $171.08,150.07,140.41,140.09,129.47,128.85,128.49,126.15,126.10,124.27,121.60$, 115.59, 59.51, 54.70, 42.56, 33.98, 23.24. HRMS calcd. for $\mathrm{C}_{19} \mathrm{H}_{21} \mathrm{~N}_{3} \mathrm{O}[\mathrm{M}+\mathrm{H}]^{+}: 308.1763$, found 308.1783 .<smiles>CN(CCc1ccccc1)Cc1ccc2ncn(C)c(=O)c2c1</smiles>

3-methyl-6-((methyl(phenethyl)amino)methyl)quinazolin-4(3H)-one (18). Following the general procedure $\mathrm{A}$, the title compound was obtained in $40 \%$ yield $(24.6 \mathrm{mg})$ as brown 
oil. ${ }^{1} \mathrm{H}$ NMR (400 MHz, $\left.\mathrm{CDCl}_{3}\right) \delta 8.20(\mathrm{~d}, J=1.5 \mathrm{~Hz}, 1 \mathrm{H}), 8.01$ (s, $\left.1 \mathrm{H}\right), 7.72(\mathrm{dd}, J=8.4$, $1.9 \mathrm{~Hz}, 0 \mathrm{H}$ ), 7.63 (d, $J=8.3 \mathrm{~Hz}, 1 \mathrm{H}), 7.29-7.25$ (m, 2H), 7.20-7.16 (m, 3H), 3.67 (s, 2H), $3.03(\mathrm{~s}, 3 \mathrm{H}), 2.83(\mathrm{dd}, J=9.3,6.2 \mathrm{~Hz}, 1 \mathrm{H}), 2.68(\mathrm{dd}, J=9.2,6.1 \mathrm{~Hz}, 1 \mathrm{H}), 2.29(\mathrm{~s}, 3 \mathrm{H}) .{ }^{13} \mathrm{C}$ NMR (101 MHz, $\left.\mathrm{CDCl}_{3}\right) \delta 161.73,147.63,146.56,140.52,139.13,135.23,128.85,128.45$, $127.63,126.31,126.10,121.73,61.81,59.32,42.25,34.15,34.10$. HRMS calcd. for $\mathrm{C}_{19} \mathrm{H}_{21} \mathrm{~N}_{3} \mathrm{O}[\mathrm{M}+\mathrm{H}]^{+}: 308.1763$, found 308.1790 .

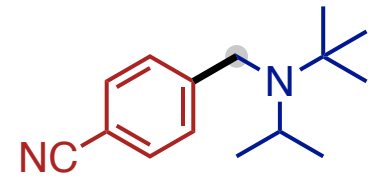

4-((tert-butyl(isopropyl)amino)methyl)benzonitrile (19). Following the general procedure $\mathrm{B}$, the title compound was obtained in $86 \%$ yield $(39.6 \mathrm{mg})$ as colorless oil. ${ }^{1} \mathrm{H}$ NMR (400 MHz, $\left.\mathrm{CDCl}_{3}\right) \delta$ 7.56-7.51 (m, 4H), 3.79 (s, 2H), 3.50 (hept, $J=6.6 \mathrm{~Hz}, 1 \mathrm{H}$ ), 1.05 (s, 9H), 0.99 (d, $J=6.6 \mathrm{~Hz}, 6 \mathrm{H}) .{ }^{13} \mathrm{C}$ NMR $\left(101 \mathrm{MHz}, \mathrm{CDCl}_{3}\right) \delta 152.77,131.89$, 131.87, 127.65, 119.53, 109.47, 55.88, 47.01, 46.14, 28.93, 22.46. HRMS calcd. for $\mathrm{C}_{15} \mathrm{H}_{22} \mathrm{~N}_{2}[\mathrm{M}+\mathrm{H}]^{+}:$231.1861, found 231.1874.

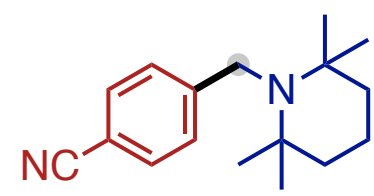

4-((2,2,6,6-tetramethylpiperidin-1-yl)methyl)benzonitrile (20). Following the general procedure $\mathrm{B}$, the title compound was obtained in $91 \%$ yield $(46.6 \mathrm{mg})$ as white solid. ${ }^{1} \mathrm{H}$ NMR (400 MHz, $\left.\mathrm{CDCl}_{3}\right) \delta$ 7.58-7.53 (m, 4H), $3.83(\mathrm{~s}, 2 \mathrm{H}), 1.62(\mathrm{br}, 2 \mathrm{H}), 1.54-1.51$ (d, $J$ $=10.8 \mathrm{~Hz}, 4 \mathrm{H}), 0.97(\mathrm{~s}, 12 \mathrm{H}) .{ }^{13} \mathrm{C}$ NMR $\left(101 \mathrm{MHz}, \mathrm{CDCl}_{3}\right) \delta$ 152.33, 131.80, 127.57, 119.56, 109.35, 55.01, 48.16, 41.28, 17.84. HRMS calcd. for $\mathrm{C}_{17} \mathrm{H}_{24} \mathrm{~N}_{2}[\mathrm{M}+\mathrm{H}]^{+}$: 257.2018, found 257.2024 .

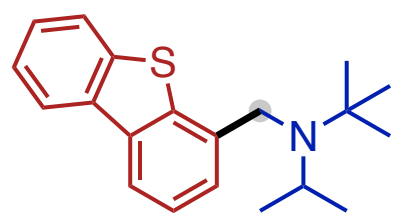

$N$-(dibenzo $[b, d]$ thiophen-4-ylmethyl)- $N$-isopropyl-2-methylpropan-2-amine (21).

Following the general procedure B, the title compound was obtained in $61 \%$ yield (38.0 $\mathrm{mg}$ ) as yellow oil. ${ }^{1} \mathrm{H} \mathrm{NMR}\left(400 \mathrm{MHz}, \mathrm{CDCl}_{3}\right) \delta 8.18-8.16(\mathrm{~m}, 1 \mathrm{H}), 8.02(\mathrm{~d}, J=7.8 \mathrm{~Hz}$, 1H), 7.92-7.89 (m, 1H), 7.77 (d, $J=7.4 \mathrm{~Hz}, 1 \mathrm{H}), 7.48-7.45$ (m, 3H), 4.01 (s, 2H), 3.60 
(hept, $J=6.7 \mathrm{~Hz}, 1 \mathrm{H}), 1.17$ (s, 9H), $1.10(\mathrm{~d}, J=6.6 \mathrm{~Hz}, 6 \mathrm{H}) .{ }^{13} \mathrm{C} \mathrm{NMR}\left(101 \mathrm{MHz}, \mathrm{CDCl}_{3}\right)$ $\delta 140.30,139.79,136.49,136.04,135.54,126.50,125.60,124.58,124.34,122.88,121.66$, 119.23, 56.06, 47.32, 45.40, 28.71, 22.45. HRMS calcd. for $\mathrm{C}_{20} \mathrm{H}_{25} \mathrm{NS}[\mathrm{M}+\mathrm{H}]^{+}: 312.1786$, found 312.1783 .

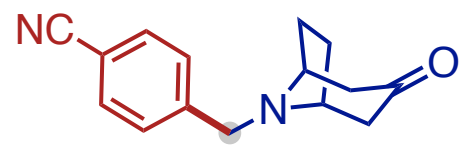

4-(((1R,5S)-3-oxo-8-azabicyclo[3.2.1]octan-8-yl)methyl)benzonitrile (22). Following the general procedure A, the title compound was obtained in $61 \%$ yield $(29.3 \mathrm{mg})$ as brown oil. ${ }^{1} \mathrm{H}$ NMR (400 MHz, $\mathrm{CDCl}_{3}$ ) $\delta$ 7.63-7.53 (m, 4H), 3.79 (s, 2H), 3.45 (d, $J=5.3 \mathrm{~Hz}$, $2 \mathrm{H}), 2.66$ (dd, $J=16.0,4.4 \mathrm{~Hz}, 2 \mathrm{H}), 2.22$ (d, $J=15.9 \mathrm{~Hz}, 2 \mathrm{H}), 2.16-2.03$ (m, 2H), 1.65 (d, $J=7.9 \mathrm{~Hz}, 2 \mathrm{H}) .{ }^{13} \mathrm{C} \mathrm{NMR}\left(101 \mathrm{MHz}, \mathrm{CDCl}_{3}\right) \delta 209.63,145.22,132.34,129.02,118.98$, 111.08, 59.03, 55.19, 48.47, 27.89. HRMS calcd. for $\mathrm{C}_{15} \mathrm{H}_{16} \mathrm{~N}_{2} \mathrm{O}[\mathrm{M}+\mathrm{H}]^{+}: 241.1341$, found 241.1345 .

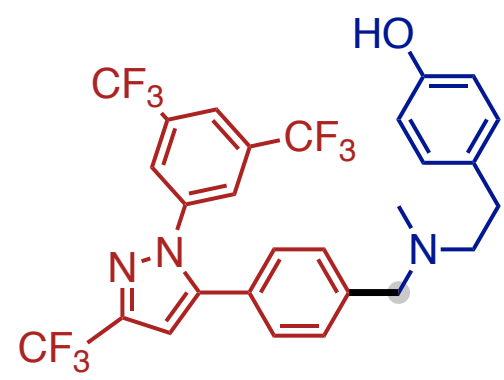

4-(2-((4-(1-(3,5-bis(trifluoromethyl)phenyl)-3-(trifluoromethyl)-1 H-pyrazol-5yl)benzyl)(methyl)amino)ethyl)phenol (23). Following the general procedure A (0.1 mmol scale), the title compound was obtained in $74 \%$ yield $\left(43.4 \mathrm{mg}\right.$ ) as colorless oil. ${ }^{1} \mathrm{H}$ NMR (400 MHz, CDCl $) \delta 7.83(\mathrm{~s}, 1 \mathrm{H}), 7.78(\mathrm{~s}, 2 \mathrm{H}), 7.35(\mathrm{~d}, J=8.1 \mathrm{~Hz}, 2 \mathrm{H}), 7.16(\mathrm{~d}, J$ $=8.2 \mathrm{~Hz}, 2 \mathrm{H}), 7.01(\mathrm{~d}, J=8.5 \mathrm{~Hz}, 2 \mathrm{H}), 6.79(\mathrm{~s}, 1 \mathrm{H}), 6.71(\mathrm{~d}, J=8.5 \mathrm{~Hz}, 2 \mathrm{H}), 3.62(\mathrm{~s}, 2 \mathrm{H})$, $2.77(\mathrm{dd}, J=9.6,5.9 \mathrm{~Hz}, 2 \mathrm{H}), 2.65(\mathrm{dd}, J=9.6,5.8 \mathrm{~Hz}, 2 \mathrm{H}), 2.27$ (s, 3H). ${ }^{13} \mathrm{C}$ NMR $(101$ $\left.\mathrm{MHz}, \mathrm{CDCl}_{3}\right) \delta 154.34,145.35,144.72(\mathrm{q}, J=38.9 \mathrm{~Hz}), 140.37,132.73(\mathrm{q}, J=34.2 \mathrm{~Hz})$, 131.98, 130.07, 129.90, 129.00, 127.18, 125.10, 125.06, 122.65 (q, $J=273.0 \mathrm{~Hz}), 121.65$, 121.03 (q, $J=269.3 \mathrm{~Hz}), 115.50,106.93,61.69,59.53,41.77,32.82 .{ }^{19} \mathrm{~F}$ NMR $(376 \mathrm{MHz}$, $\left.\mathrm{CDCl}_{3}\right) \delta-62.55,-63.15$. HRMS calcd. for $\mathrm{C}_{28} \mathrm{H}_{22} \mathrm{~N}_{3} \mathrm{OF}_{9}[\mathrm{M}+\mathrm{H}]^{+}: 588.1697$, found 588.1683. 


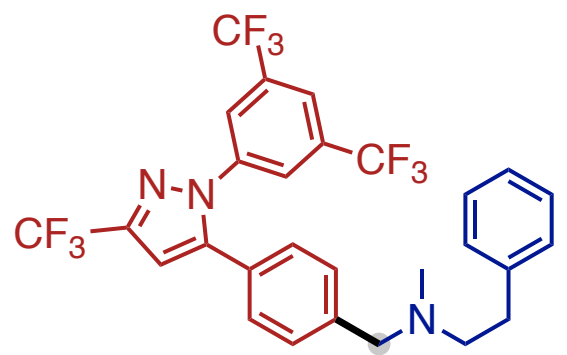

\section{$N$-(4-(1-(3,5-bis(trifluoromethyl)phenyl)-3-(trifluoromethyl)-1H-pyrazol-5-}

yl)benzyl)- $N$-methyl-2-phenylethan-1-amine (24). Following the general procedure A ( $0.1 \mathrm{mmol}$ scale), the title compound was obtained in $84 \%$ yield $(48.0 \mathrm{mg})$ as colorless oil. ${ }^{1} \mathrm{H}$ NMR $\left(400 \mathrm{MHz}, \mathrm{CDCl}_{3}\right) \delta 7.82(\mathrm{~s}, 1 \mathrm{H}), 7.78(\mathrm{~s}, 2 \mathrm{H}), 7.32$ (d, J=8.1 Hz, 2H), 7.28$7.24(\mathrm{~m}, 2 \mathrm{H}), 7.20-7.13(\mathrm{~m}, 5 \mathrm{H}), 6.78(\mathrm{~s}, 1 \mathrm{H}), 3.58(\mathrm{~s}, 2 \mathrm{H}), 2.85-2.81(\mathrm{~m}, 2 \mathrm{H}), 2.66$ (dd, $J$ $=9.0,6.3 \mathrm{~Hz}, 2 \mathrm{H}), 2.26(\mathrm{~s}, 3 \mathrm{H}) \cdot{ }^{13} \mathrm{C} \mathrm{NMR}\left(101 \mathrm{MHz}, \mathrm{CDCl}_{3}\right) \delta 145.49,144.74(\mathrm{q}, J=38.9$ $\mathrm{Hz}), 141.81,140.53,140.47,132.77$ (q, $J=34.1 \mathrm{~Hz}), 129.75,128.95,128.89,128.46$, 126.97, 126.15, 125.10, 122.69 (q, $J=273.2 \mathrm{~Hz}), 121.61,121.07$ (q, $J=269.2 \mathrm{~Hz}), 106.90$, 61.83, 59.38, 42.04, 34.10. ${ }^{19} \mathrm{~F}$ NMR (376 MHz, $\left.\mathrm{CDCl}_{3}\right) \delta-62.57,-63.17$. HRMS calcd. for $\mathrm{C}_{28} \mathrm{H}_{22} \mathrm{~N}_{3} \mathrm{~F}_{9}[\mathrm{M}+\mathrm{H}]^{+}: 572.1748$, found 572.1742 .

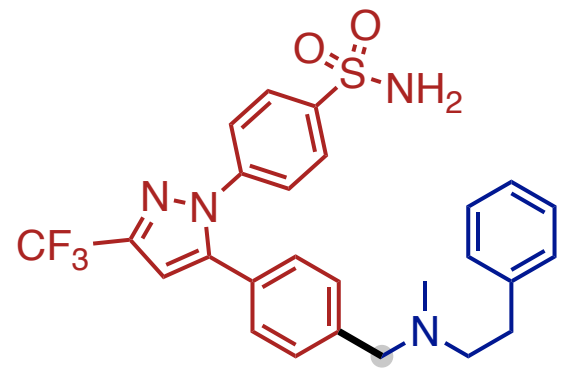

4-(5-(4-((methyl(phenethyl)amino)methyl)phenyl)-3-(trifluoromethyl)-1H-pyrazol-1yl)benzenesulfonamide (25). Following the general procedure A (0.1 mmol scale), the title compound was obtained in $65 \%$ yield $(33.4 \mathrm{mg})$ as brown oil. ${ }^{1} \mathrm{H}$ NMR $(400 \mathrm{MHz}$, $\left.\mathrm{CDCl}_{3}\right) \delta 7.86(\mathrm{~d}, J=8.8 \mathrm{~Hz}, 1 \mathrm{H}), 7.44(\mathrm{~d}, J=8.8 \mathrm{~Hz}, 1 \mathrm{H}), 7.30-7.25$ (m, 4H), 7.20-7.14 (m, 5H), $6.76(\mathrm{~s}, 1 \mathrm{H}), 5.14(\mathrm{br}, 1 \mathrm{H}), 3.57(\mathrm{~s}, 2 \mathrm{H}), 2.81(\mathrm{dd}, J=9.2,6.2 \mathrm{~Hz}, 2 \mathrm{H}), 2.66$ (dd, $J=9.3,6.2 \mathrm{~Hz}, 2 \mathrm{H}), 2.29(\mathrm{~s}, 3 \mathrm{H}) .{ }^{13} \mathrm{C} \mathrm{NMR}\left(101 \mathrm{MHz}, \mathrm{CDCl}_{3}\right) \delta 145.20,144.25(\mathrm{q}, J=$ $38.6 \mathrm{~Hz}), 142.56,141.66,140.87,140.34,129.70,128.90,128.84,128.52,127.59,127.43$, 126.22, 125.60, 121.16 (q, $J=269.3 \mathrm{~Hz}), 106.64,61.72,59.27,42.31,33.86 .{ }^{19} \mathrm{~F}$ NMR (376 MHz, $\left.\mathrm{CDCl}_{3}\right) \delta-62.39$. HRMS calcd. for $\mathrm{C}_{26} \mathrm{H}_{25} \mathrm{~N}_{4} \mathrm{O}_{2} \mathrm{SF}_{3}[\mathrm{M}+\mathrm{H}]^{+}: 515.1729$, found 515.1731 . 


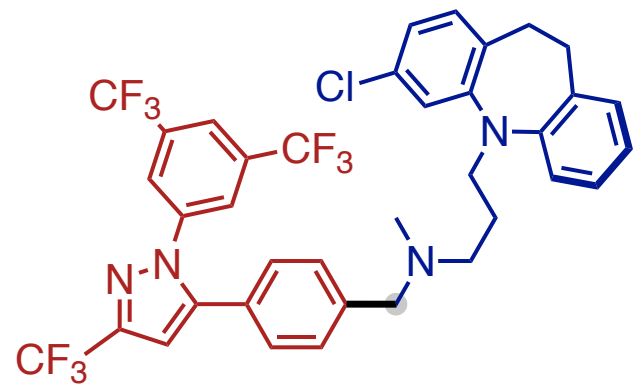

\section{$\mathrm{N}$-(4-(1-(3,5-bis(trifluoromethyl)phenyl)-3-(trifluoromethyl)-1H-pyrazol-5-}

\section{yl)benzyl)-3-(3-chloro-10,11-dihydro-5H-dibenzo[b,f]azepin-5-yl)- $N$-methylpropan-}

1-amine (26). Following the general procedure A ( $0.1 \mathrm{mmol}$ scale), the title compound was obtained in $68 \%$ yield $(50.1 \mathrm{mg})$ as yellow oil. ${ }^{1} \mathrm{H}$ NMR $\left(400 \mathrm{MHz}, \mathrm{CDCl}_{3}\right) \delta 7.84(\mathrm{~s}$, $1 \mathrm{H}), 7.79(\mathrm{~s}, 2 \mathrm{H}), 7.29-7.27(\mathrm{~m}, 2 \mathrm{H}), 7.16-7.05(\mathrm{~m}, 6 \mathrm{H}), 6.97-6.94(\mathrm{~m}, 2 \mathrm{H}), 6.85$ (dd, $J=$ 8.1, $2.1 \mathrm{~Hz}, 1 \mathrm{H}), 6.80(\mathrm{~s}, 1 \mathrm{H}), 3.77$ (t, $J=6.7 \mathrm{~Hz}, 2 \mathrm{H}), 3.47$ (s, 2H), 3.10-3.03 (m, 4H), $2.44(\mathrm{t}, J=6.6 \mathrm{~Hz}, 2 \mathrm{H}), 2.12(\mathrm{~s}, 3 \mathrm{H}), 1.80-1.74(\mathrm{~m}, 2 \mathrm{H}) .{ }^{13} \mathrm{C} \mathrm{NMR}\left(101 \mathrm{MHz}, \mathrm{CDCl}_{3}\right) \delta$ 149.21, 148.01, 145.45, 144.74 (q, $J=38.9 \mathrm{~Hz}), 140.46,135.24,132.75$ (q, $J=34.1 \mathrm{~Hz}$ ), $131.69,131.63,131.32,129.74,129.57,128.94,126.95,126.70,125.10,123.37,122.69$ (q, $J=273.1 \mathrm{~Hz}), 122.07,121.64,121.07$ (q, $J=269.5 \mathrm{~Hz}), 120.68,119.96,106.84,61.98$, 55.10, 48.75, 41.98, 32.35, 31.68, 25.76. ${ }^{19} \mathrm{~F}$ NMR (376 MHz, $\left.\mathrm{CDCl}_{3}\right) \delta-62.53,-63.13$. HRMS calcd. for $\mathrm{C}_{37} \mathrm{H}_{30} \mathrm{~N}_{4} \mathrm{ClF}_{9}[\mathrm{M}+\mathrm{H}]^{+}:$737.2094, found 737.2083.

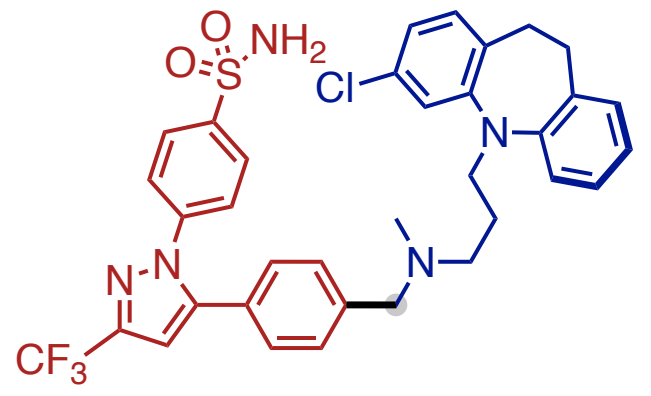

\section{4-(5-(4-((3-(3-chloro-10,11-dihydro-5H-dibenzo[b,f]azepin-5-}

\section{yl)propyl)(methyl)amino)methyl)phenyl)-3-(trifluoromethyl)-1H-pyrazol-1-}

yl)benzenesulfonamide (27). Following the general procedure A ( $0.1 \mathrm{mmol}$ scale), the title compound was obtained in 51\% yield $(34.6 \mathrm{mg})$ as brown oil. ${ }^{1} \mathrm{H}$ NMR (400 MHz, $\left.\mathrm{CDCl}_{3}\right) \delta 7.88(\mathrm{~d}, J=8.8 \mathrm{~Hz}, 2 \mathrm{H}), 7.45(\mathrm{~d}, J=8.7 \mathrm{~Hz}, 2 \mathrm{H}), 7.24-7.22(\mathrm{~m}, 2 \mathrm{H}), 7.12-7.02$ (m, 6H), 6.96-6.92 (m, 2H), 6.84 (dd, $J=8.1,2.0 \mathrm{~Hz}, 1 \mathrm{H}), 6.77$ (s, 1H), $5.10(\mathrm{br}, 2 \mathrm{H}), 3.75$ (t, $J=6.6 \mathrm{~Hz}, 2 \mathrm{H}), 3.45$ (s, 2H), 3.04 (s, 4H), 2.43 (t, $J=6.4 \mathrm{~Hz}, 2 \mathrm{H}), 2.14(\mathrm{~s}, 3 \mathrm{H}), 1.75$ 
(p, $J=6.5 \mathrm{~Hz}, 2 \mathrm{H}) \cdot{ }^{19} \mathrm{~F}$ NMR $\left(376 \mathrm{MHz}, \mathrm{CDCl}_{3}\right) \delta-62.39 .{ }^{13} \mathrm{C} \mathrm{NMR}\left(101 \mathrm{MHz}, \mathrm{CDCl}_{3}\right) \delta$ $148.99,147.77,145.07,144.13$ (q, $J=38.6 \mathrm{~Hz}), 142.44,141.58,135.10,131.49,131.25$, $129.54,129.46,128.82,128.74,127.50,127.43,127.26,126.60,125.53,123.30,121.96$, 121.06 (q, $J=269.2 \mathrm{~Hz}), 120.53,119.76,106.47,61.75,54.94,48.60,42.18,32.18,31.48$, 25.41. ${ }^{19} \mathrm{~F}$ NMR (376 MHz, $\left.\mathrm{CDCl}_{3}\right) \delta$-62.36. HRMS calcd. for $\mathrm{C}_{35} \mathrm{H}_{33} \mathrm{~N}_{5} \mathrm{O}_{2} \mathrm{SClF}_{3}[\mathrm{M}+\mathrm{H}]^{+}$: 680.2074 , found 680.2067 .

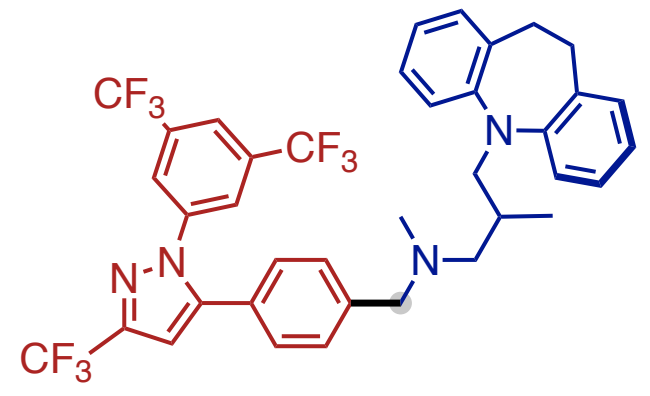

$N$-(4-(1-(3,5-bis(trifluoromethyl)phenyl)-3-(trifluoromethyl)-1H-pyrazol-5-

yl)benzyl)-3-(10,11-dihydro-5H-dibenzo $[b, f]$ azepin-5-yl)- $N$,2-dimethylpropan-1-

amine (28). Following the general procedure A ( $0.1 \mathrm{mmol}$ scale), the title compound was obtained in $47 \%$ yield $\left(34.0 \mathrm{mg}\right.$ ) as brown oil. ${ }^{1} \mathrm{H}$ NMR $\left(400 \mathrm{MHz}, \mathrm{CDCl}_{3}\right) \delta 7.82(\mathrm{~s}, 1 \mathrm{H})$, 7.79 (s, 2H), 7.38 (d, $J=7.6 \mathrm{~Hz}, 2 \mathrm{H}), 7.17$ (d, $J=7.9 \mathrm{~Hz}, 2 \mathrm{H}), 7.10-7.08$ (m, 6H), 6.92$6.88(\mathrm{~m}, 2 \mathrm{H}), 6.81(\mathrm{~s}, 1 \mathrm{H}), 3.99-3.94(\mathrm{~m}, 1 \mathrm{H}), 3.49-4.40(\mathrm{~m}, 2 \mathrm{H}), 3.29-3.14(\mathrm{~m}, 2 \mathrm{H}), 3.18$ (s, 2H), 2.39-2.32 (m, 1H), 2.18-2.01 (m, 5H), 0.93 (d, $J=5.9 \mathrm{~Hz}, 3 \mathrm{H}) .{ }^{13} \mathrm{C}$ NMR (101 $\left.\mathrm{MHz}, \mathrm{CDCl}_{3}\right) \delta 148.60,145.34,144.60(\mathrm{~d}, J=38.8 \mathrm{~Hz}), 142.08,140.32,134.14,132.61$ (d, $J=34.3 \mathrm{~Hz}), 129.88,129.55,128.79,126.26,124.95,124.92,122.55$ (d, $J=273.1 \mathrm{~Hz})$, 122.34, 121.50, 120.94 (d, $J=269.3 \mathrm{~Hz}), 119.87,106.80,106.79,63.14,62.40,56.00$, 42.74, 32.30, 29.44, 17.51. ${ }^{19} \mathrm{~F}$ NMR (376 MHz, $\left.\mathrm{CDCl}_{3}\right) \delta-62.56,-63.13$. HRMS calcd. for $\mathrm{C}_{38} \mathrm{H}_{33} \mathrm{~N}_{4} \mathrm{~F}_{9}[\mathrm{M}+\mathrm{H}]^{+}$: 717.2640, found 717.2653.

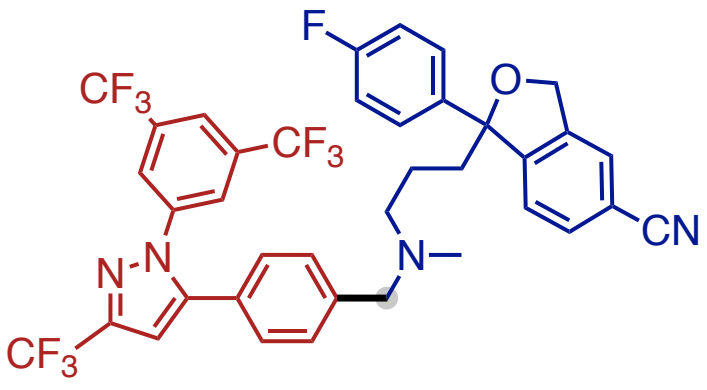

1-(3-((4-(1-(3,5-bis(trifluoromethyl)phenyl)-3-(trifluoromethyl)-1H-pyrazol-5yl)benzyl)(methyl)amino)propyl)-1-(4-fluorophenyl)-1,3-dihydroisobenzofuran-5- 
carbonitrile (29). Following the general procedure A ( $0.1 \mathrm{mmol} \mathrm{scale})$, the title compound was obtained in $45 \%$ yield $\left(31.9 \mathrm{mg}\right.$ ) as colorless oil. ${ }^{1} \mathrm{H} \mathrm{NMR}\left(400 \mathrm{MHz}, \mathrm{CDCl}_{3}\right) \delta 7.82$ (s, 1H), 7.77 (s, 2H), 7.59 (d, $J=7.9 \mathrm{~Hz}, 1 \mathrm{H}), 7.51(\mathrm{~s}, 1 \mathrm{H}), 7.44-7.37$ (m, 3H), 7.32 (d, $J=$ $8.0 \mathrm{~Hz}, 2 \mathrm{H}), 7.16$ (d, $J=8.1 \mathrm{~Hz}, 2 \mathrm{H}), 7.00$ (t, $J=8.7 \mathrm{~Hz}, 2 \mathrm{H}), 2.36$ (t, $J=6.8 \mathrm{~Hz}, 2 \mathrm{H})$, $7.69(\mathrm{~s}, 1 \mathrm{H}), 5.21-5.13(\mathrm{~m}, 2 \mathrm{H}), 3.45(\mathrm{~s}, 2 \mathrm{H}), 2.27-2.10(\mathrm{~m}, 2 \mathrm{H}), 2.08(\mathrm{~s}, 3 \mathrm{H}), 1.58-1.48$ (m, 1H), 1.44-1.35 (m, 1H). ${ }^{13} \mathrm{C}$ NMR (101 MHz, $\left.\mathrm{CDCl}_{3}\right) \delta 163.41,160.96,149.59,145.33$, 144.71 (q, $J=38.8 \mathrm{~Hz}), 140.50,140.38,139.66,132.68$ (q, $J=34.2 \mathrm{~Hz}), 131.99,129.82$, $128.97,126.90,126.82,125.38,125.03,125.00,122.83,122.65$ (d, $J=273.1 \mathrm{~Hz}), 121.58$, 121.01 (d, $J=269.2 \mathrm{~Hz}), 118.73,115.59,115.37,111.92,106.91,91.23,71.39,61.87$, 57.27, 41.77, 38.98, 21.98, 14.32. ${ }^{19} \mathrm{~F}$ NMR $\left(376 \mathrm{MHz}, \mathrm{CDCl}_{3}\right) \delta-62.56,-63.14,-115.31$. HRMS calcd. for $\mathrm{C}_{38} \mathrm{H}_{28} \mathrm{~N}_{4} \mathrm{OF}_{10}[\mathrm{M}+\mathrm{H}]^{+}$: 747.2181, found 747.2181.

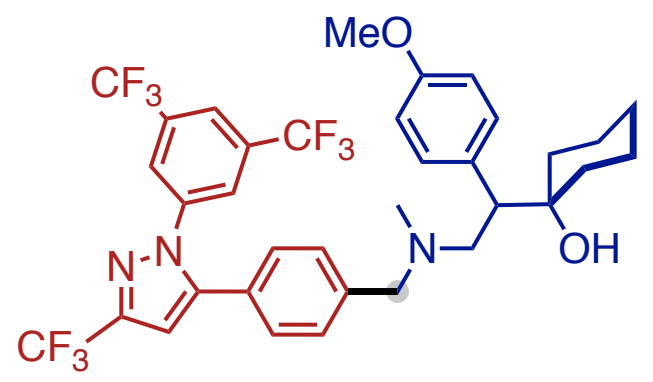

\section{1-(2-((4-(1-(3,5-bis(trifluoromethyl)phenyl)-3-(trifluoromethyl)-1 H-pyrazol-5-}

yl)benzyl)(methyl)amino)-1-(4-methoxyphenyl)ethyl)cyclohexan-1-ol (30). Following the general procedure A $(0.1 \mathrm{mmol}$ scale), the title compound was obtained in $83 \%$ yield (58.0 mg) as colorless oil. ${ }^{1} \mathrm{H}$ NMR $\left(400 \mathrm{MHz}, \mathrm{CDCl}_{3}\right) \delta 7.84(\mathrm{~s}, 1 \mathrm{H}), 7.78(\mathrm{~s}, 2 \mathrm{H}), 7.37$ (d, $J=7.9 \mathrm{~Hz}, 2 \mathrm{H}), 7.21(\mathrm{~d}, J=8.1 \mathrm{~Hz}, 2 \mathrm{H}), 7.03$ (d, $J=8.6 \mathrm{~Hz}, 2 \mathrm{H}), 6.81$ (d, $J=10.1 \mathrm{~Hz}$, $3 \mathrm{H}), 3.79-3.78(\mathrm{~m}, 4 \mathrm{H}), 3.47-3.37(\mathrm{~m}, 2 \mathrm{H}), 3.07-3.04(\mathrm{~m}, 1 \mathrm{H}), 2.47-2.43(\mathrm{~m}, 1 \mathrm{H}), 2.26(\mathrm{~s}$, $3 \mathrm{H}), 1.71-0.83(\mathrm{~m}, 11 \mathrm{H}) .{ }^{13} \mathrm{C} \mathrm{NMR}\left(101 \mathrm{MHz}, \mathrm{CDCl}_{3}\right) \delta 74.40,62.31,59.37,55.18,51.55$, 41.93, 37.95, 31.25, 25.84, 21.48, 21.34. ${ }^{13} \mathrm{C}$ NMR (101 MHz, $\left.\mathrm{CDCl}_{3}\right) \delta 158.40,145.04$, $144.61(\mathrm{q}, J=38.8 \mathrm{~Hz}), 140.24,140.05,132.67$ (q, $J=34.3 \mathrm{~Hz}), 132.53,130.10,129.01$, 127.40, 125.06, 125.03, 122.51 (q, $J=273.2 \mathrm{~Hz}), 121.65(\mathrm{~m}), 120.87$ (q, $J=269.3 \mathrm{~Hz})$, $113.39,106.85,74.40,62.31,59.37,55.18,51.55,41.93,37.95,31.25,25.84,21.48,21.34$. ${ }^{19} \mathrm{~F}$ NMR (376 MHz, $\left.\mathrm{CDCl}_{3}\right) \delta-62.56,-63.16$. HRMS calcd. for $\mathrm{C}_{35} \mathrm{H}_{34} \mathrm{~N}_{3} \mathrm{O}_{2} \mathrm{~F}_{9}[\mathrm{M}+\mathrm{H}]^{+}$: 700.2585 , found 700.2574 . 


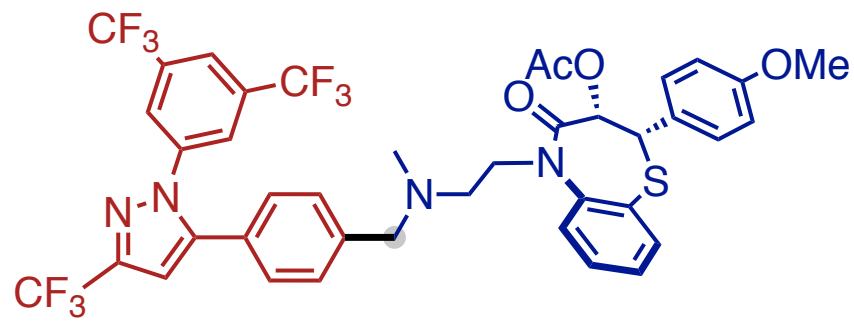

(contains 19\% oxidized diltiazem impurity, likely the formamide)

(2S,3S)-5-(2-((4-(1-(3,5-bis(trifluoromethyl)phenyl)-3-(trifluoromethyl)-1H-pyrazol5-yl)benzyl)(methyl)amino)ethyl)-2-(4-methoxyphenyl)-4-0xo-2,3,4,5-

tetrahydrobenzo[b][1,4]thiazepin-3-yl acetate (31). Following the general procedure A (0.1 mmol scale), the title compound was obtained in $77 \%$ yield $(64.0 \mathrm{mg})$ as colorless oil. ${ }^{1} \mathrm{H}$ NMR $\left(400 \mathrm{MHz}, \mathrm{CDCl}_{3}\right) \delta 7.83(\mathrm{~s}, 1 \mathrm{H}), 7.78(\mathrm{~s}, 2 \mathrm{H}), 7.71(\mathrm{~d}, J=7.4 \mathrm{~Hz}, 1 \mathrm{H}), 7.48-$ 7.47 (m, 2H), 7.44-7.41 (m, 2H), 7.28-7.24 (3H), 7.12 (d, $J=8.1 \mathrm{~Hz}, 2 \mathrm{H}), 6.87$ (d, $J=8.7$ $\mathrm{Hz}, 2 \mathrm{H}), 6.80(\mathrm{~s}, 1 \mathrm{H}), 5.18(\mathrm{~d}, J=7.6 \mathrm{~Hz}, 1 \mathrm{H}), 5.03$ (d, $J=7.6 \mathrm{~Hz}, 1 \mathrm{H}), 4.51$ (dt, $J=13.7$, $6.8 \mathrm{~Hz}, 1 \mathrm{H}), 3.83-3.79(\mathrm{~m}, 1 \mathrm{H}), 3.81(\mathrm{~s}, 3 \mathrm{H}), 3.63-3.54(\mathrm{~m}, 2 \mathrm{H}), 2.92-2.85(\mathrm{~m}, 1 \mathrm{H}), 2.75-$ $2.68(\mathrm{~m}, 1 \mathrm{H}), 2.17$ (s, 3H), 1.91 (s, 3H). ${ }^{13} \mathrm{C}$ NMR $\left(101 \mathrm{MHz}, \mathrm{CDCl}_{3}\right) \delta$ 170.02, 167.09, $159.86,145.82,145.41,144.64$ (q, $J=38.8 \mathrm{~Hz}), 140.86,140.37,135.53,132.68$ (q, $J=$ $34.2 \mathrm{~Hz}), 131.10,130.91,129.82$, 128.85, 127.40, 126.78, 125.04, 125.00, 124.70, 123.23, $122.62(\mathrm{q}, J=273.1 \mathrm{~Hz}), 121.58,121.02(\mathrm{q}, J=269.3 \mathrm{~Hz}), 113.84,106.86,71.21,61.79$, 55.63, 55.28, 54.57, 47.88, 41.82, 20.59. ${ }^{19} \mathrm{~F}$ NMR (376 MHz, $\left.\mathrm{CDCl}_{3}\right) \delta-62.55,-63.16$. HRMS calcd. for $\mathrm{C}_{40} \mathrm{H}_{33} \mathrm{~N}_{4} \mathrm{O}_{4} \mathrm{SF}_{9}[\mathrm{M}+\mathrm{H}]^{+}:$837.2157, found 837.2169.

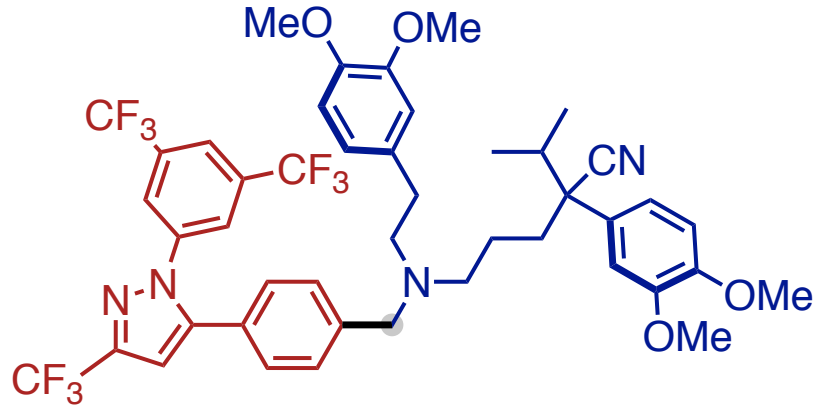

\section{5-((4-(1-(3,5-bis(trifluoromethyl)phenyl)-3-(trifluoromethyl)-1 $H$-pyrazol-5-} yl)benzyl)(3,4-dimethoxyphenethyl)amino)-2-(3,4-dimethoxyphenyl)-2-

isopropylpentanenitrile (32). Following the general procedure $\mathrm{A}(0.1 \mathrm{mmol}$ scale), the title compound was obtained in $64 \%$ yield $(56.1 \mathrm{mg})$ as colorless oil. ${ }^{1} \mathrm{H}$ NMR (400 MHz, $\left.\mathrm{CDCl}_{3}\right) \delta 7.83(\mathrm{~s}, 1 \mathrm{H}), 7.78(\mathrm{~s}, 2 \mathrm{H}), 7.28-7.26(\mathrm{~m}, 2 \mathrm{H}), 7.14(\mathrm{~d}, J=7.7 \mathrm{~Hz}, 2 \mathrm{H}), 6.87-6.80$ 
(m, 4H), $6.76(\mathrm{~d}, J=8.3 \mathrm{~Hz}, 1 \mathrm{H}), 6.63-6.61(\mathrm{~m}, 2 \mathrm{H}), 3.89$ (d, $J=2.9 \mathrm{~Hz}, 1 \mathrm{H}), 3.85(\mathrm{~s}, 3 \mathrm{H})$, $3.84(\mathrm{~s}, 6 \mathrm{H}), 3.81(\mathrm{~s}, 3 \mathrm{H}), 3.57(\mathrm{br}, 1 \mathrm{H}), 2.64-1.50(\mathrm{~m}, 11 \mathrm{H}), 1.15(\mathrm{~d}, J=6.6 \mathrm{~Hz}, 3 \mathrm{H}), 0.78$ $(\mathrm{d}, J=6.7 \mathrm{~Hz}, 3 \mathrm{H}) .{ }^{13} \mathrm{C} \mathrm{NMR}\left(101 \mathrm{MHz}, \mathrm{CDCl}_{3}\right) \delta 149.20,148.99,148.52,147.54,145.39$, $144.63(\mathrm{q}, J=39.3 \mathrm{~Hz}), 142.31,140.44,133.09,132.58$ (q, $J=34.6,34.0 \mathrm{~Hz}), 130.80$, $129.52,128.86,126.88,125.05,122.54$ (q, $J=273.0 \mathrm{~Hz}), 121.54,120.88(\mathrm{q}, J=269.2 \mathrm{~Hz})$, 120.74, 118.75, 112.34, 111.45, 111.30, 110.00, 106.95, 58.07, 56.13, 56.07, 55.98, 55.54, $53.45,53.05,38.10,35.62,33.17,23.20,19.02,18.65 .{ }^{19} \mathrm{~F} \mathrm{NMR}\left(376 \mathrm{MHz}, \mathrm{CDCl}_{3}\right) \delta$ 62.57, -63.08. HRMS calcd. for $\mathrm{C}_{45} \mathrm{H}_{45} \mathrm{~N}_{4} \mathrm{O}_{4} \mathrm{~F}_{9}[\mathrm{M}+\mathrm{H}]^{+}$: 877.3375, found 877.3352.

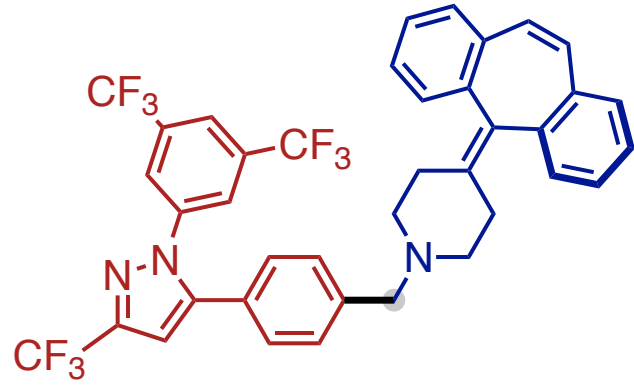

1-(4-(1-(3,5-bis(trifluoromethyl)phenyl)-3-(trifluoromethyl)-1H-pyrazol-5yl)benzyl)-4-(5H-dibenzo[a,d][7]annulen-5-ylidene)piperidine (33). Following the general procedure A ( $0.1 \mathrm{mmol}$ scale), the title compound was obtained in $45 \%$ yield (31.9 $\mathrm{mg})$ as red oil. ${ }^{1} \mathrm{H} \mathrm{NMR}\left(400 \mathrm{MHz}, \mathrm{CDCl}_{3}\right) \delta 7.74(\mathrm{~s}, 1 \mathrm{H}), 7.68(\mathrm{~s}, 2 \mathrm{H}), 7.30-7.22(\mathrm{~m}, 6 \mathrm{H})$, 7.18-7.07 (m, 6H), 6.85 (s, 2H), $6.71(\mathrm{~s}, 1 \mathrm{H}), 3.43$ (s, 2H), 2.54-2.39 (m, 2H), 2.36-2.23 $(\mathrm{m}, 2 \mathrm{H}), 2.12-2.03(\mathrm{~m}, 4 \mathrm{H}) .{ }^{13} \mathrm{C} \mathrm{NMR}\left(101 \mathrm{MHz}, \mathrm{CDCl}_{3}\right) \delta 145.36,144.72(\mathrm{q}, J=38.9$ $\mathrm{Hz}), 140.38,139.21,134.92,132.73$ (q, $J=34.3 \mathrm{~Hz}), 131.14,130.03,128.95,128.62$, 128.35, 127.91, 126.44, 125.07, 125.04, 122.64 (q, $J=273.1 \mathrm{~Hz}), 121.63,121.04$ (d, $J=$ 269.3 Hz), 106.90, 62.36, 55.16, 30.05. ${ }^{19} \mathrm{~F}$ NMR (376 MHz, $\left.\mathrm{CDCl}_{3}\right) \delta-62.56,-63.20$. HRMS calcd. for $\mathrm{C}_{39} \mathrm{H}_{28} \mathrm{~N}_{3} \mathrm{~F}_{9}[\mathrm{M}+\mathrm{H}]^{+}:$710.2218, found 710.2220.

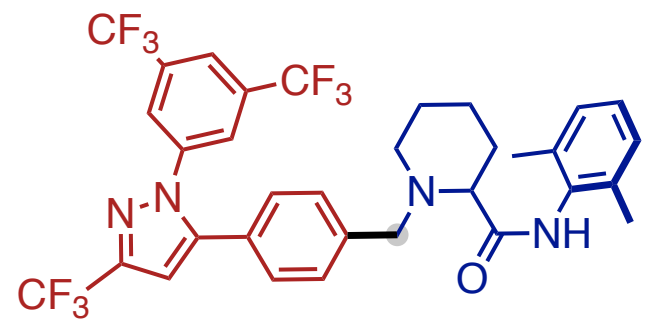

1-(4-(1-(3,5-bis(trifluoromethyl)phenyl)-3-(trifluoromethyl)-1H-pyrazol-5yl)benzyl)- $N$-(2,6-dimethylphenyl)piperidine-2-carboxamide (34). Following the 
general procedure A ( $0.1 \mathrm{mmol}$ scale), the title compound was obtained in $37 \%$ yield ( 24.7 $\mathrm{mg}$ ) as white solid. ${ }^{1} \mathrm{H}$ NMR (400 MHz, $\left.\mathrm{CDCl}_{3}\right) \delta 8.08(\mathrm{~s}, 1 \mathrm{H}), 7.82(\mathrm{~s}, 1 \mathrm{H}), 7.77(\mathrm{~s}, 2 \mathrm{H})$, $7.42(\mathrm{~d}, J=8.1 \mathrm{~Hz}, 2 \mathrm{H}), 7.21$ (d, $J=8.2 \mathrm{~Hz}, 2 \mathrm{H}), 7.12-7.05$ (m, 3H), 6.80 (s, 1H), 4.24 (d, $J=14.2 \mathrm{~Hz}, 1 \mathrm{H}$ ), 3.42 (d, $J=14.2 \mathrm{~Hz}, 1 \mathrm{H}), 3.06$ (dd, $J=10.2,3.4 \mathrm{~Hz}, 1 \mathrm{H}), 2.93$ (dt, $J=$ $11.7,3.3 \mathrm{~Hz}, 1 \mathrm{H}), 2.23-2.18(\mathrm{~m}, 1 \mathrm{H}), 2.18(\mathrm{~s}, 6 \mathrm{H}), 2.08-2.01(\mathrm{~m}, 1 \mathrm{H}), 1.88-1.78(\mathrm{~m}, 2 \mathrm{H})$, 1.71-1.67 (m, 1H), 1.54-1.37 (m, 2H). ${ }^{13} \mathrm{C}$ NMR (101 MHz, $\left.\mathrm{CDCl}_{3}\right) \delta$ 172.59, 145.11, 144.79 (q, $J=38.8 \mathrm{~Hz}), 140.34,135.60,133.56,132.78$ (q, $J=34.2 \mathrm{~Hz}), 129.30,128.59$, $127.53,127.42,125.05,125.02,122.64$ (q, $J=273.0 \mathrm{~Hz}), 121.72,121.00$ (q, $J=269.4 \mathrm{~Hz})$, $106.98,68.57,60.86,51.96,31.04,24.94,23.61,18.84 .{ }^{19} \mathrm{~F} \mathrm{NMR}\left(376 \mathrm{MHz}, \mathrm{CDCl}_{3}\right) \delta$ 62.57, -63.20. HRMS calcd. for $\mathrm{C}_{33} \mathrm{H}_{29} \mathrm{~N}_{4} \mathrm{OF}_{9}[\mathrm{M}+\mathrm{H}]^{+}$: 669.2276, found 669.2272.

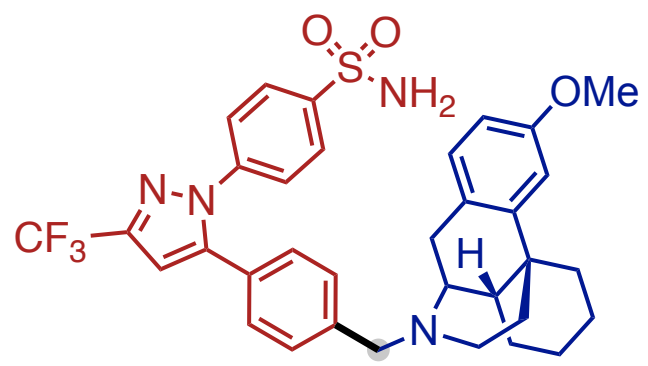

\section{4-(5-(4-(((4bS,8aS)-3-methoxy-6,7,8,8a,9,10-hexahydro-5H-9,4b-}

\section{(epiminoethano)phenanthren-11-yl)methyl)phenyl)-3-(trifluoromethyl)-1H-pyrazol-}

1-yl)benzenesulfonamide (35). Following the general procedure A $(0.1 \mathrm{mmol}$ scale), the title compound was obtained in $72 \%$ yield $(45.8 \mathrm{mg})$ as yellow oil. ${ }^{1} \mathrm{H}$ NMR (400 MHz, $\left.\mathrm{CDCl}_{3}\right) \delta 7.90-7.87(\mathrm{~m}, 2 \mathrm{H}), 7.48-7.44(\mathrm{~m}, 2 \mathrm{H}), 7.39-7.37(\mathrm{~m}, 2 \mathrm{H}), 7.16(\mathrm{~d}, J=8.2 \mathrm{~Hz}$, 2H), $7.05(\mathrm{~d}, J=8.4 \mathrm{~Hz}, 1 \mathrm{H}), 6.81(\mathrm{~d}, J=2.6 \mathrm{~Hz}, 1 \mathrm{H}), 6.76(\mathrm{~s}, 1 \mathrm{H}), 6.71(\mathrm{dd}, J=8.4,2.6$ $\mathrm{Hz}, 1 \mathrm{H}), 5.10$ (br, 2H), 3.78 (s, 3H), 3.77-3.60 (m, 2H), 3.03-3.63 (m, 3H), 2.42-2.11 (m, $3 \mathrm{H}), 1.89-1.50(\mathrm{~m}, 4 \mathrm{H}), 1.37-1.24(\mathrm{~m}, 5 \mathrm{H}), 1.16-1.07$ (m, 1H). ${ }^{13} \mathrm{C} \mathrm{NMR}\left(101 \mathrm{MHz}, \mathrm{CDCl}_{3}\right)$ $\delta 158.39,145.29,144.24$ (q, $J=38.6 \mathrm{~Hz}), 142.63,141.94,141.61,129.89,129.39,128.88$, 128.66, 127.62, 127.18, 125.64, 121.17 (q, $J=269.2 \mathrm{~Hz}), 111.28,110.91,106.60,59.02$, $56.75,55.34,45.52,45.29,42.08,37.89,36.74,26.94,26.68,24.91,22.37 .{ }^{19} \mathrm{~F}$ NMR (376 $\left.\mathrm{MHz}, \mathrm{CDCl}_{3}\right) \delta$-62.41. HRMS calcd. for $\mathrm{C}_{34} \mathrm{H}_{35} \mathrm{~N}_{4} \mathrm{O}_{3} \mathrm{SF}_{3}[\mathrm{M}+\mathrm{H}]^{+}:$637.2460, found 637.2471 . 


\section{References}

1. Loh, Y. Y.; Nagao, K.; Hoover, A. J.; Hesk, D.; Rivera, N. R.; Colletti, S. L.; Davies, I. W.; MacMillan, D. W. C. Photoredox-Catalyzed Deuteration and Tritiation of Pharmaceutical Compounds. Science 2017, 358, 1182-1187.

2. Shen, Y.; Funes-Ardoiz, I.; Schoenebeck, F.; Rovis, T. Site-selective $\alpha-C-H$ Functionalization of Trialkylamines via Reversible Hydrogen Atom Transfer Catalysis. ChemRxiv 2021, DOI: 10.26434/chemrxiv.14442290.v1.

3. Chang, Y.; Yesilcimen, A.; Cao, M.; Zhang, Y.; Zhang, B.; Chan, J. Z.; Wasa, M. Catalytic Deuterium Incorporation within Metabolically Stable $\beta$-Amino $\mathrm{C}-\mathrm{H}$ Bonds of Drug Molecules. J. Am. Chem. Soc. 2019, 141, 14570-14575. 
V. ${ }^{1} \mathrm{H},{ }^{13} \mathrm{C}$ and ${ }^{19} \mathrm{~F}$ NMR spectra ys-878.454.fid
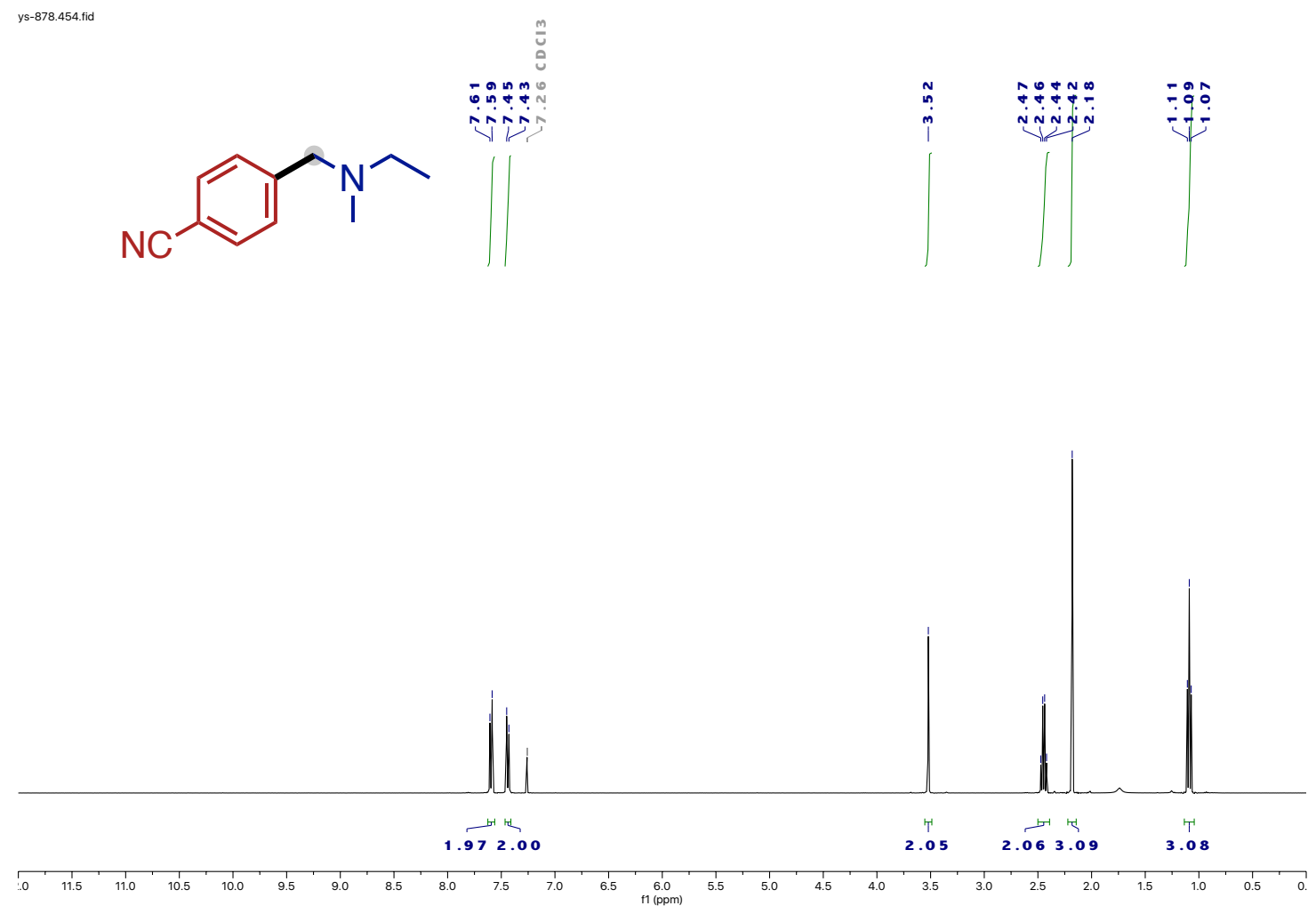

ys-878-c13.457.fid
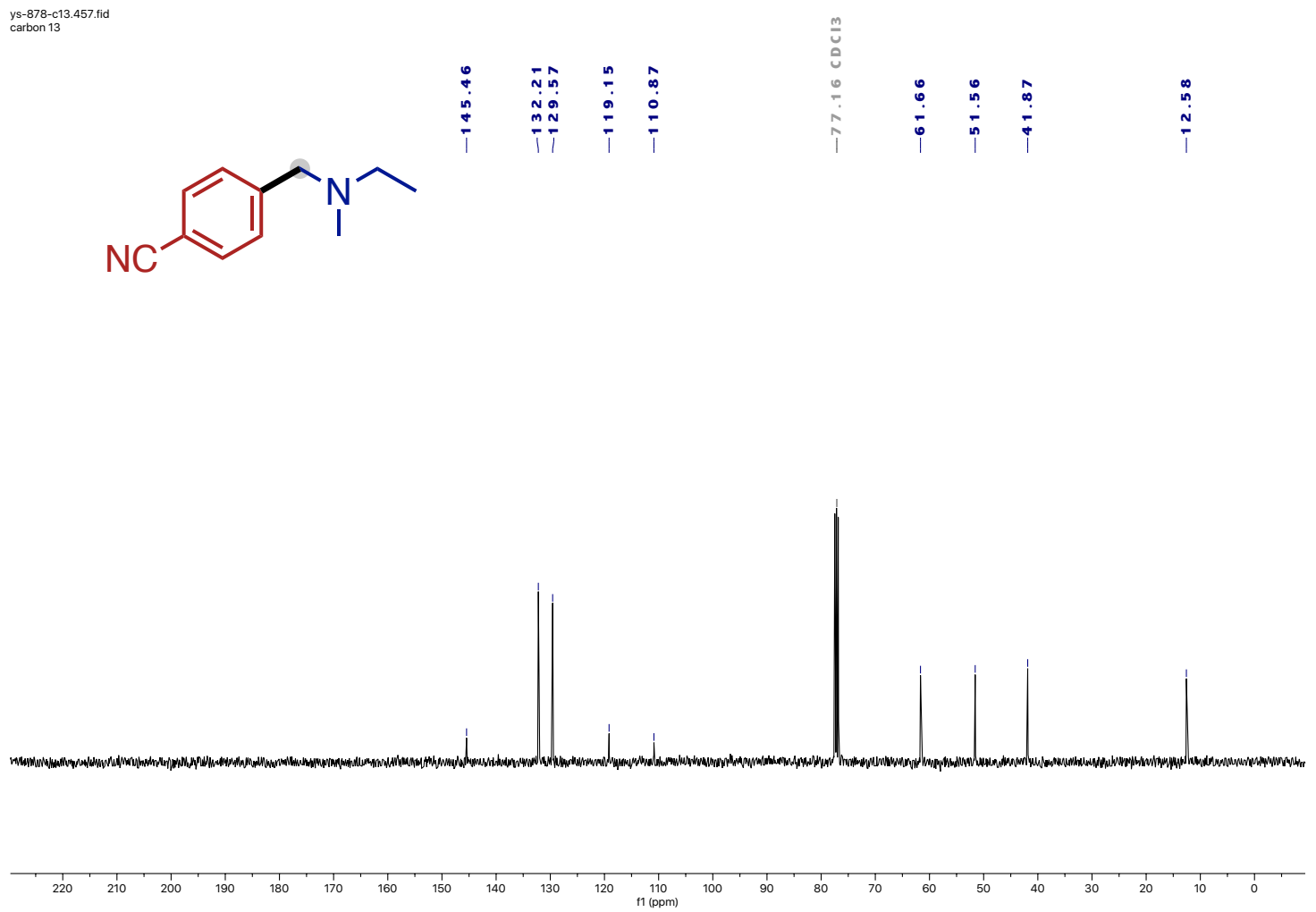


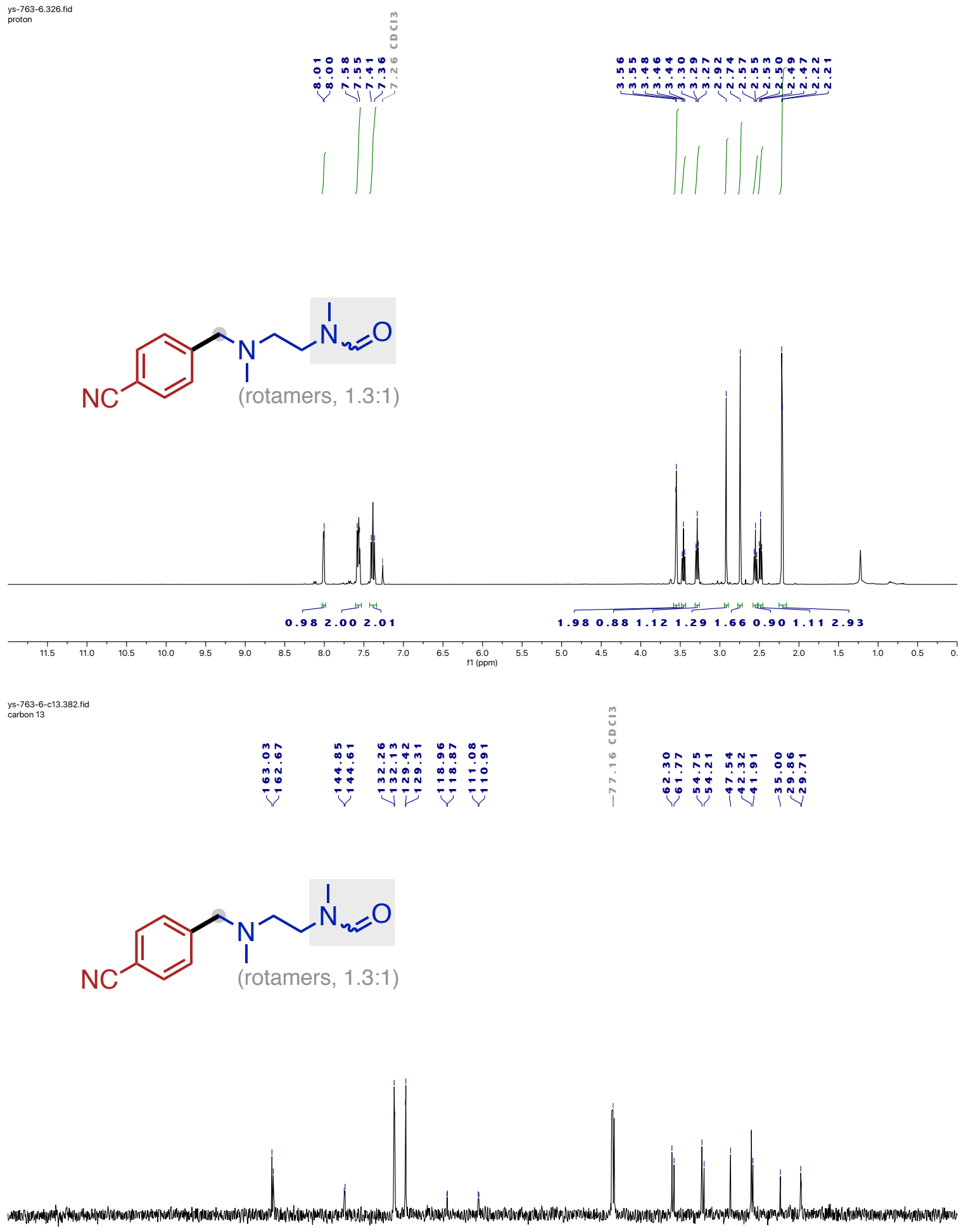

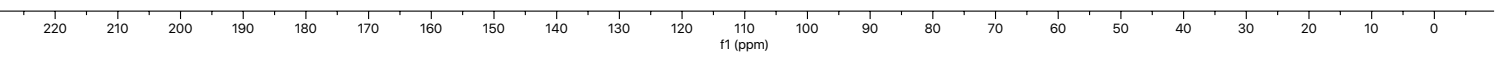




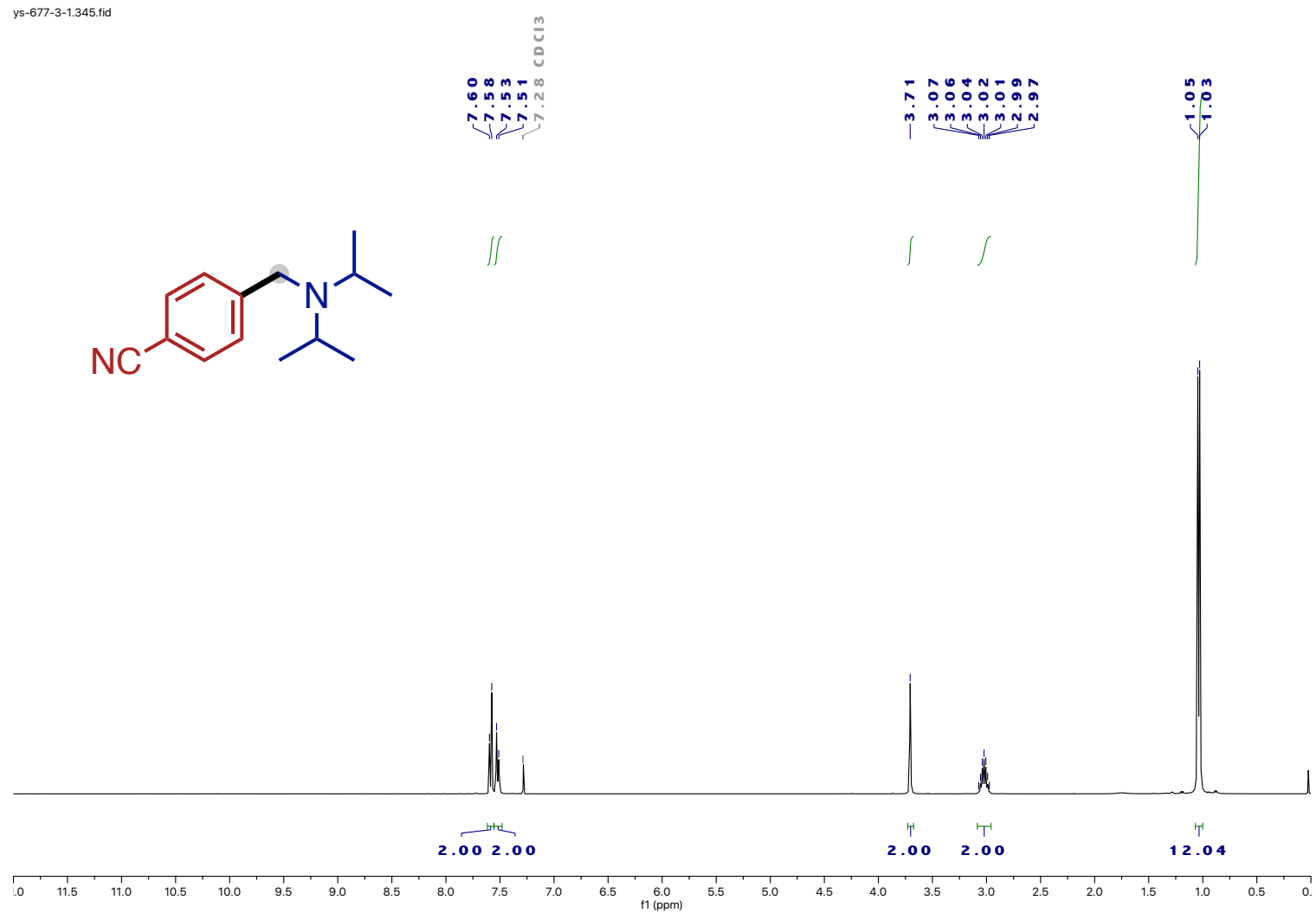

ys-677-3-3-1-c.346. fid
carbon 13

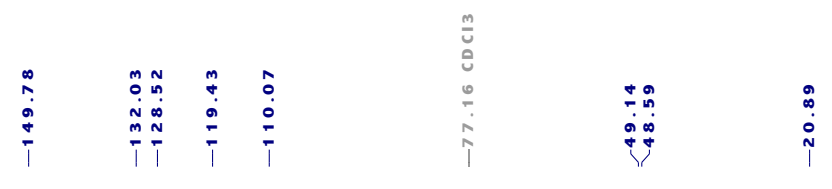<smiles>CC(C)N(Cc1ccc(C#N)cc1)C(C)C</smiles>

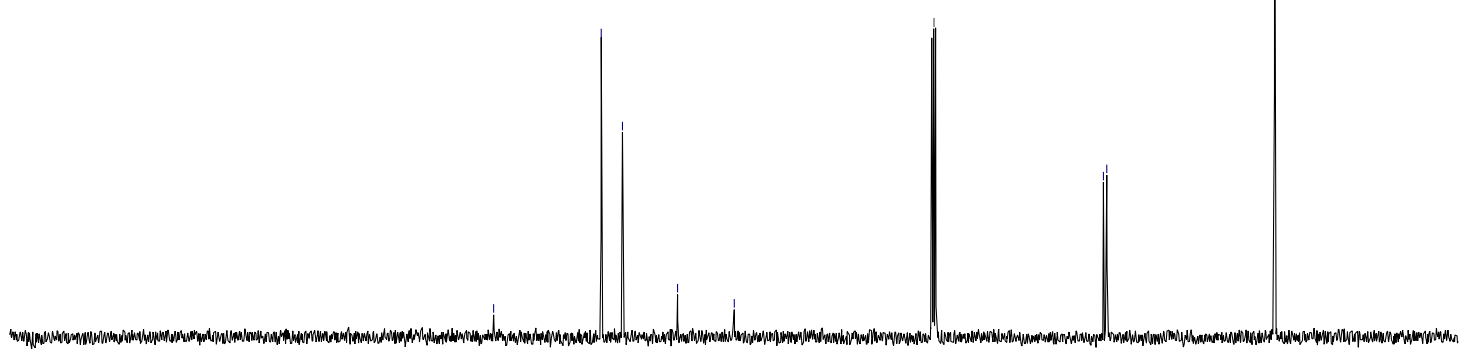



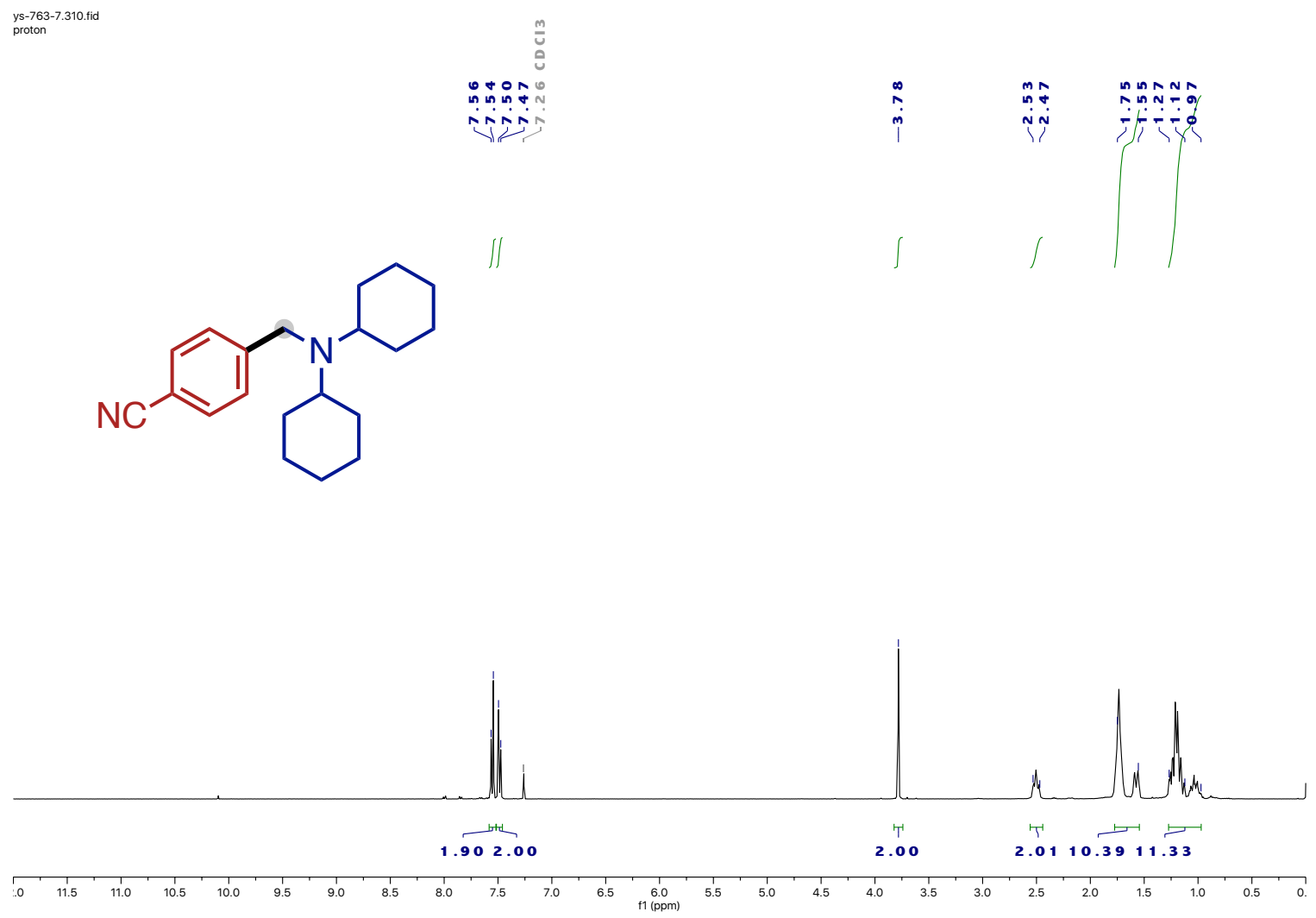

ys-763-7-c13.313.fid

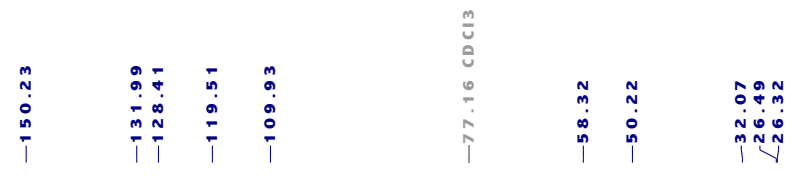

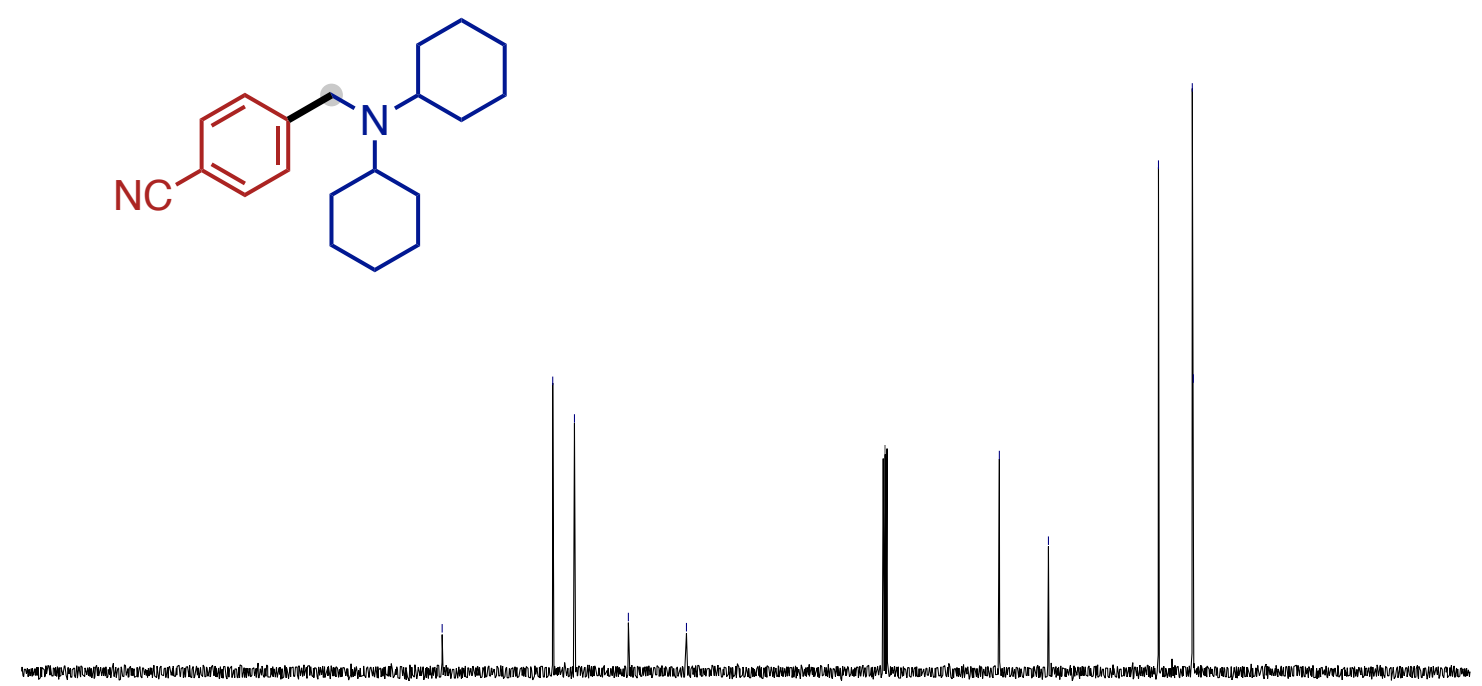




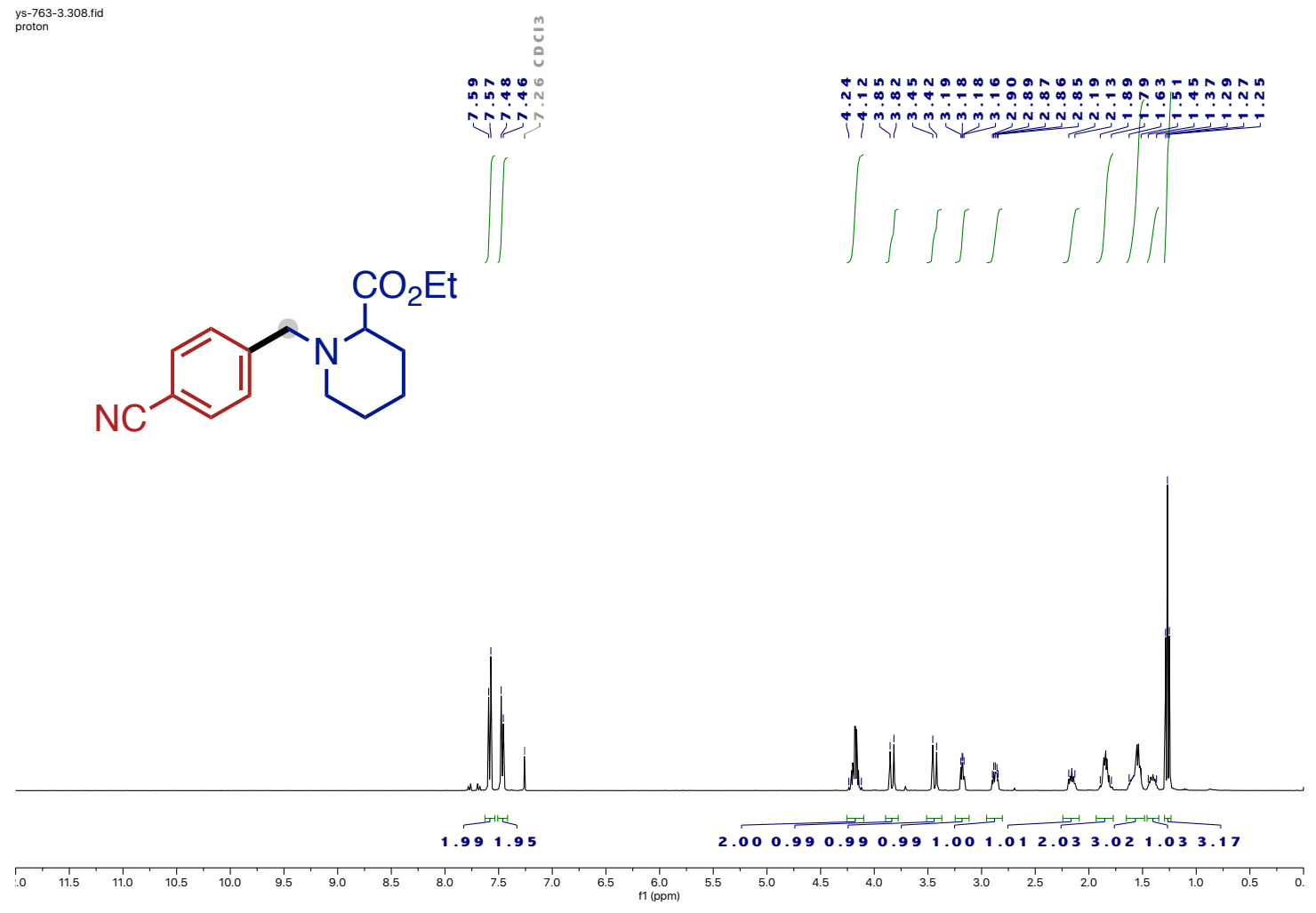

ys-763-3-c13.315.fid

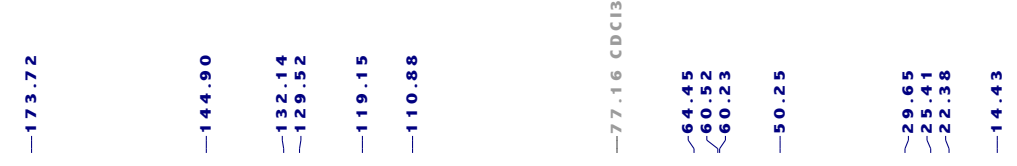
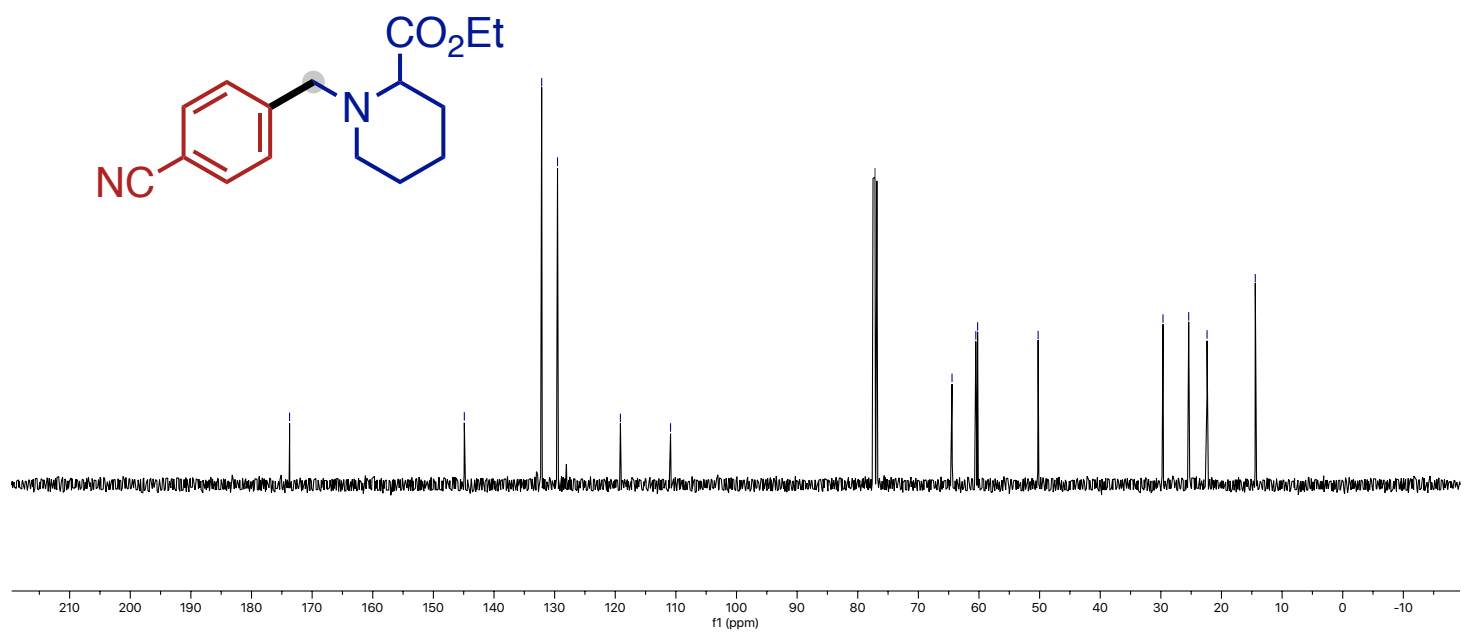


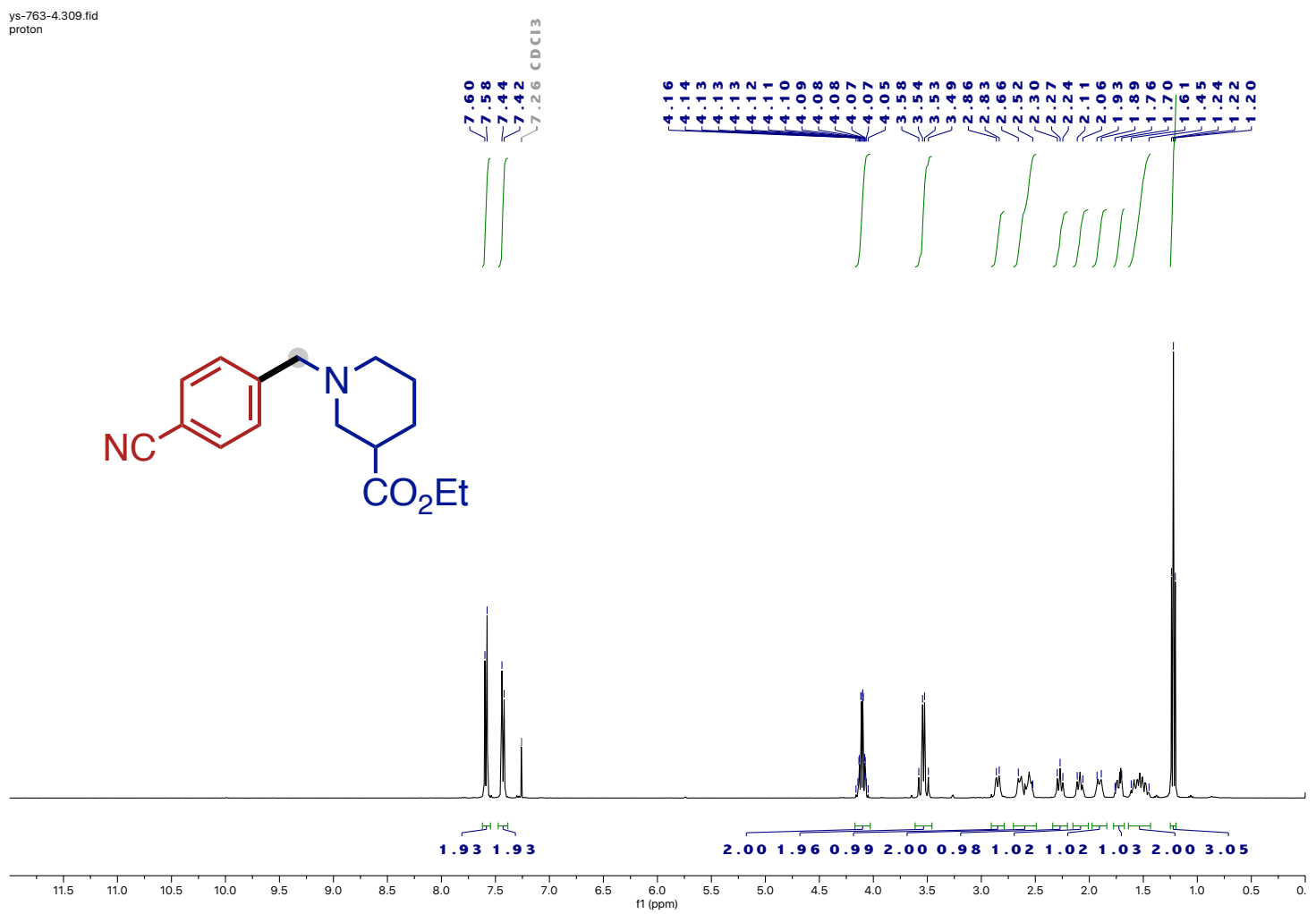

ys-763-4-c13.314.fid

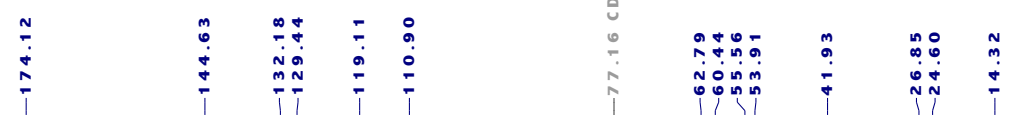<smiles>CCOC(=O)C1CCCN(Cc2ccc(C#N)cc2)C1</smiles>
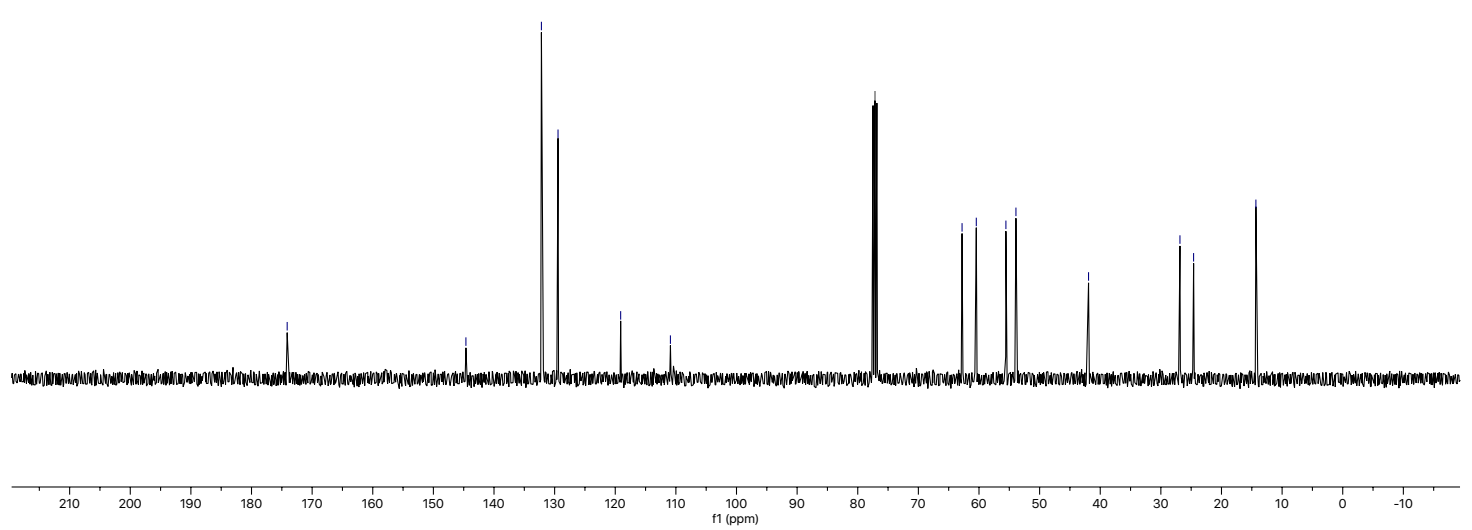


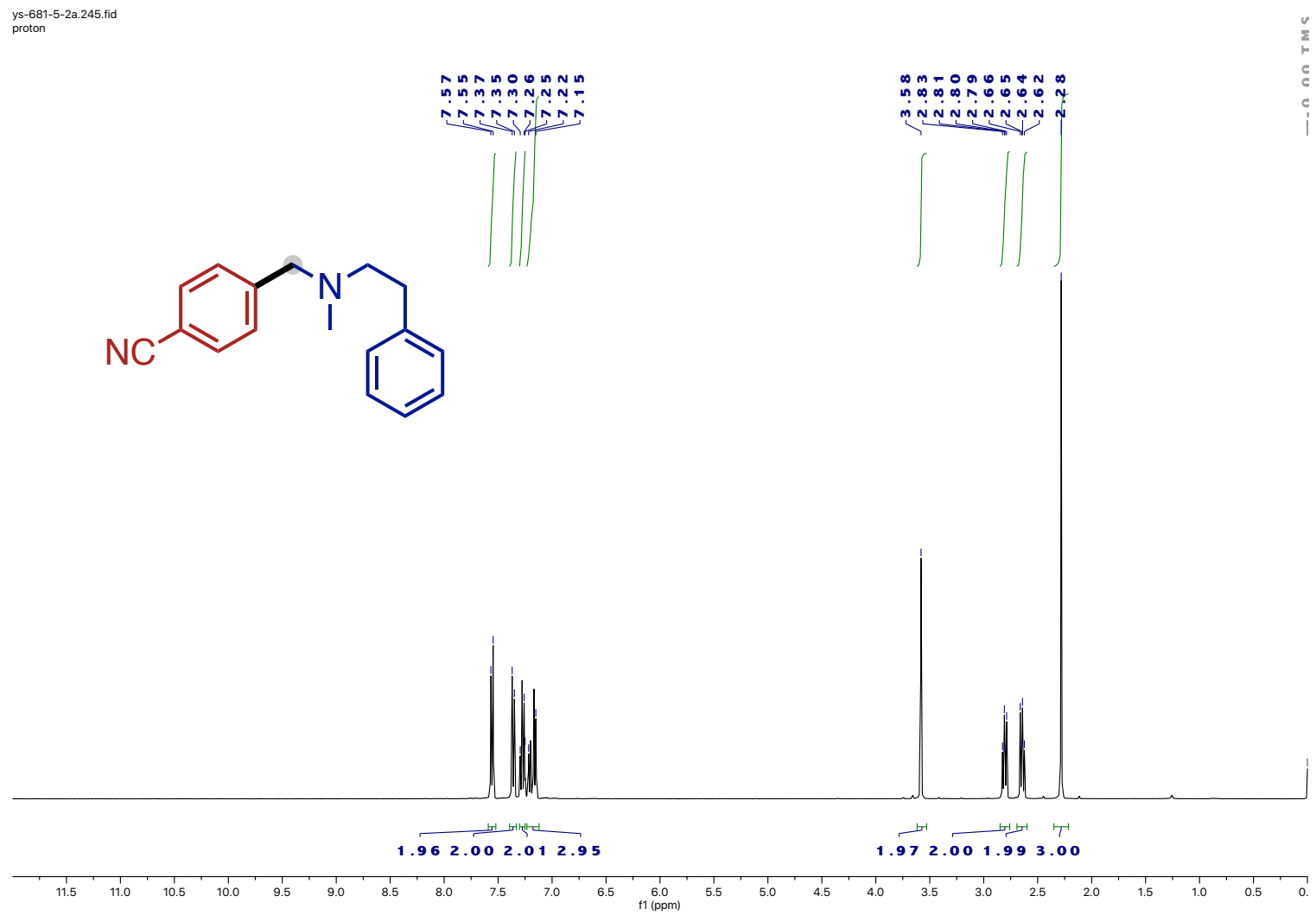

ys-681-5-2a-c.246.fid

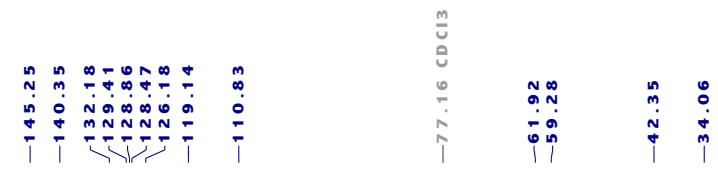<smiles>CN(CCc1ccccc1)Cc1ccc(C#N)cc1</smiles>

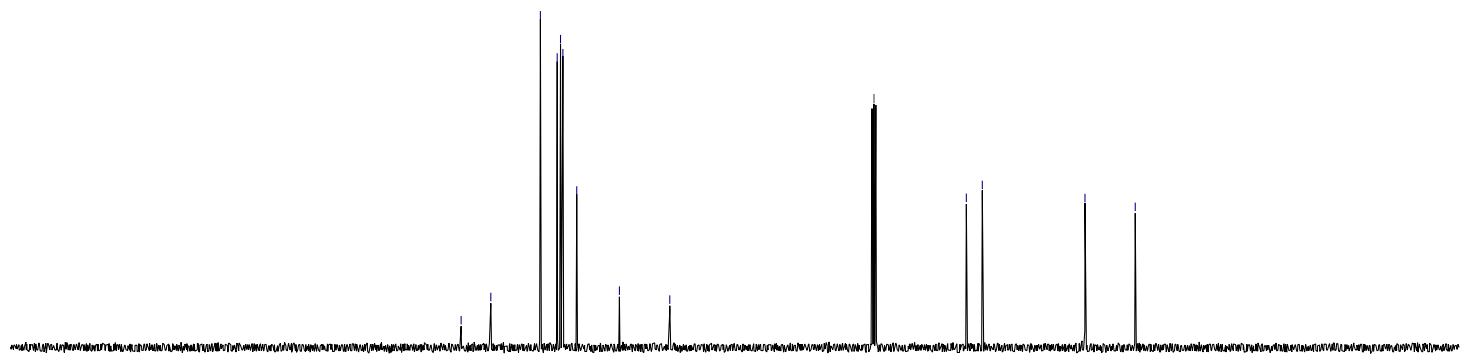




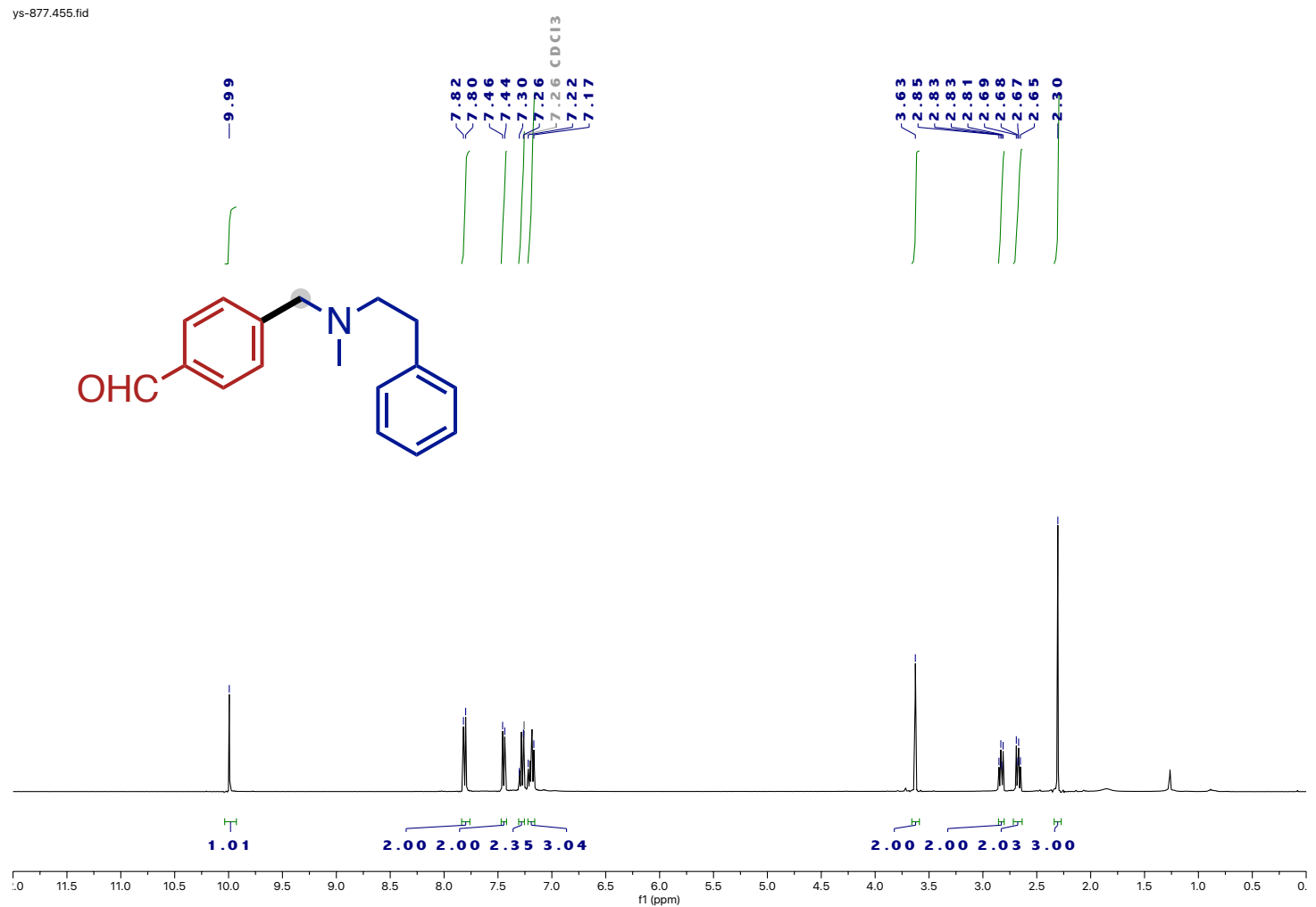

ys-877-c13.456.fid
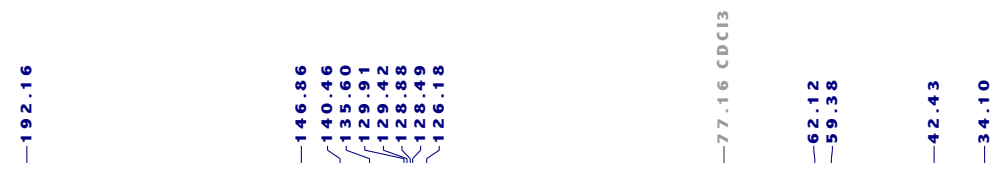<smiles>CN(CCc1ccccc1)Cc1ccc(C=O)cc1</smiles>
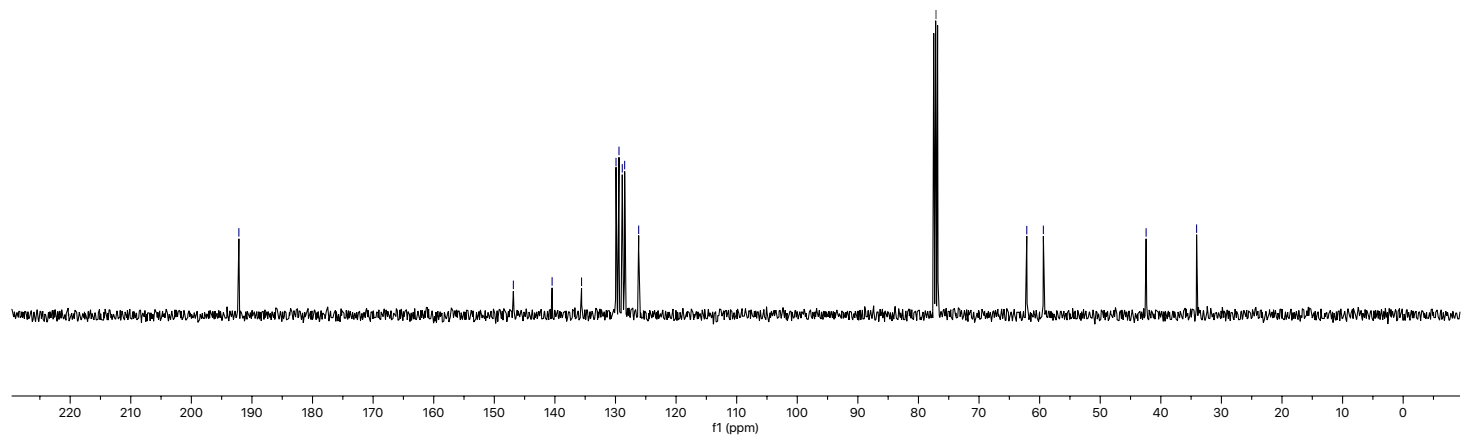

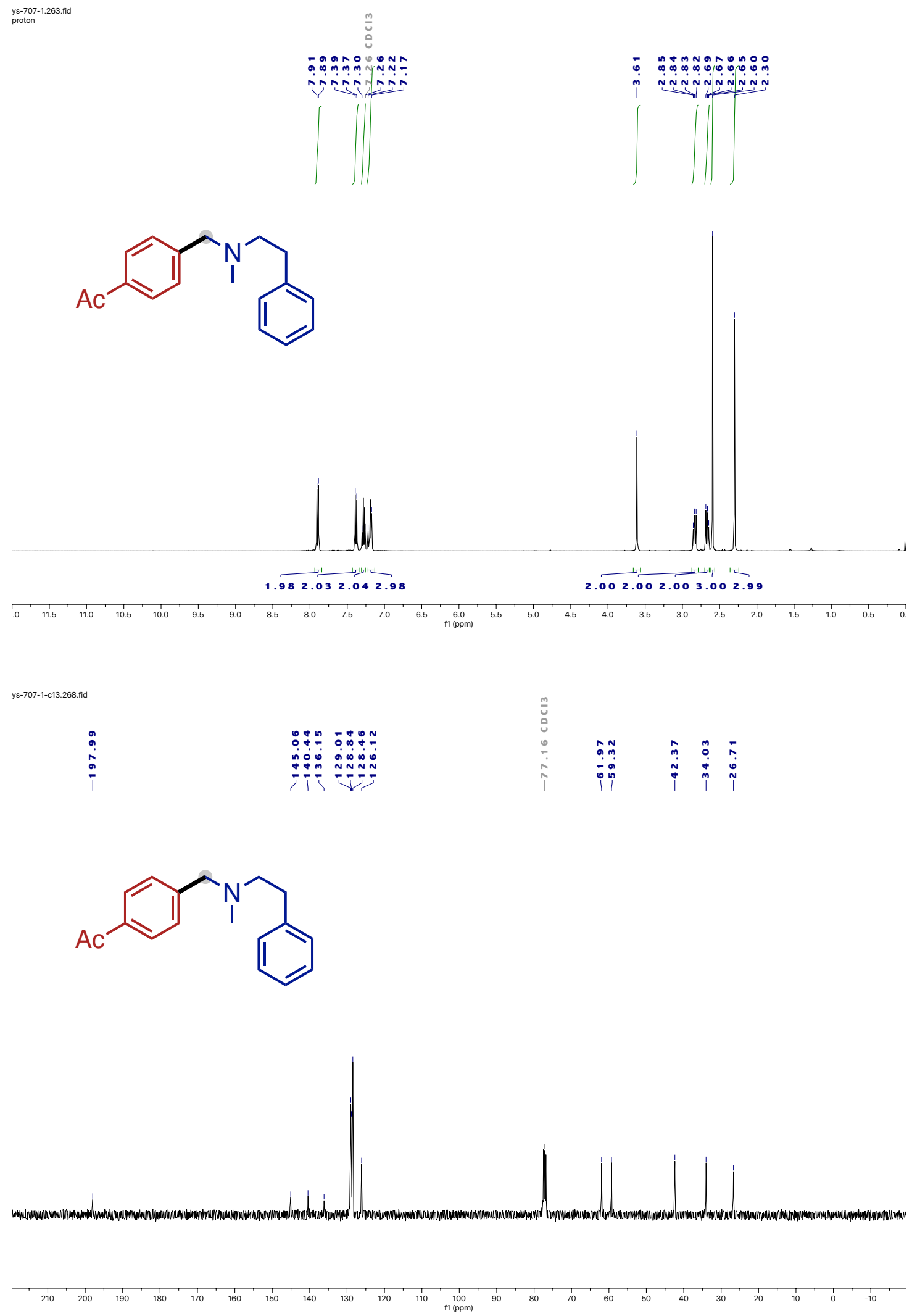

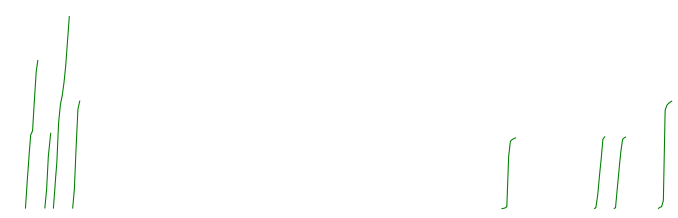<smiles>CN(CCc1ccccc1)Cc1ccc(-c2ccccc2)cc1</smiles>

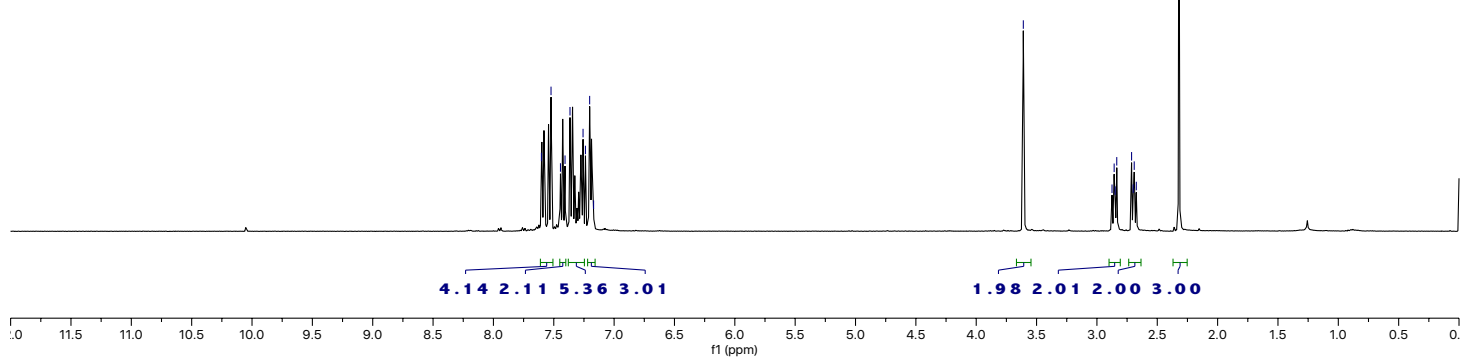

ys-728-c.296.fid
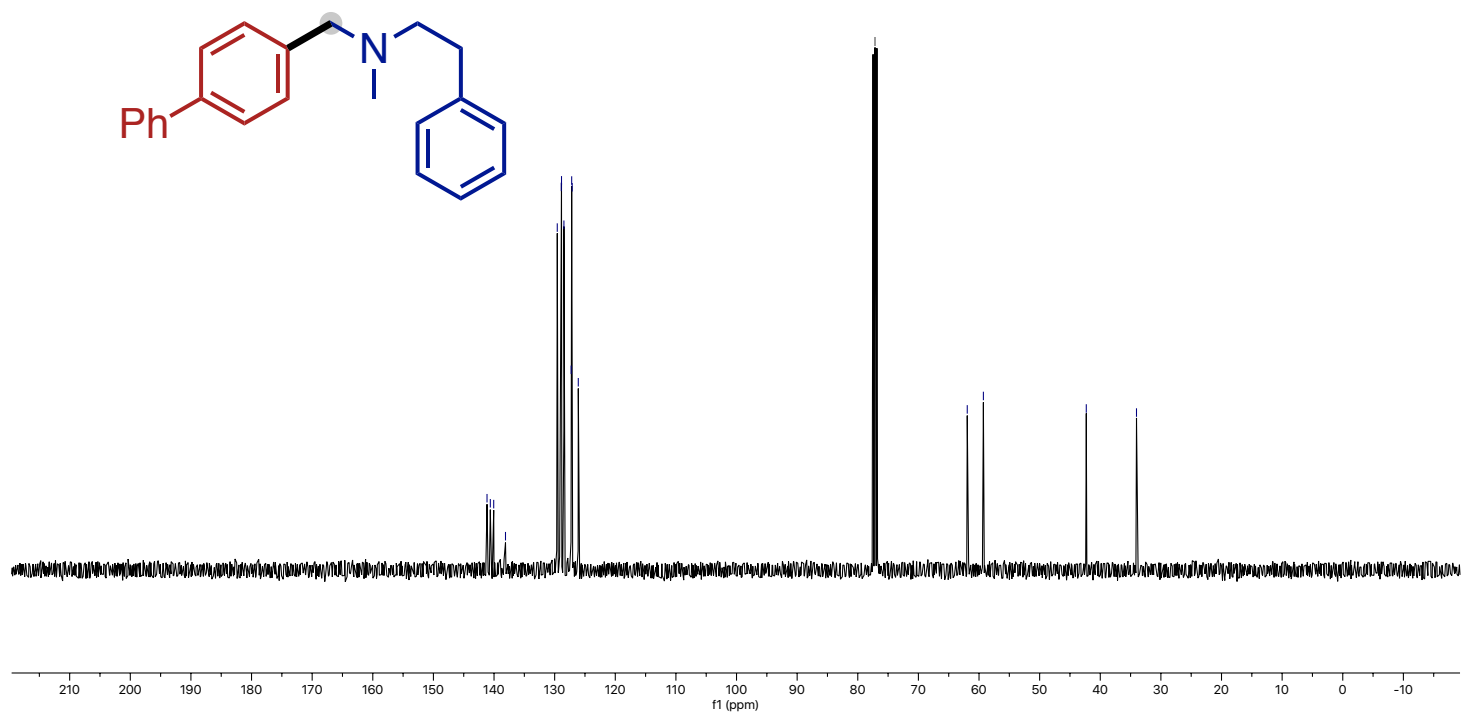


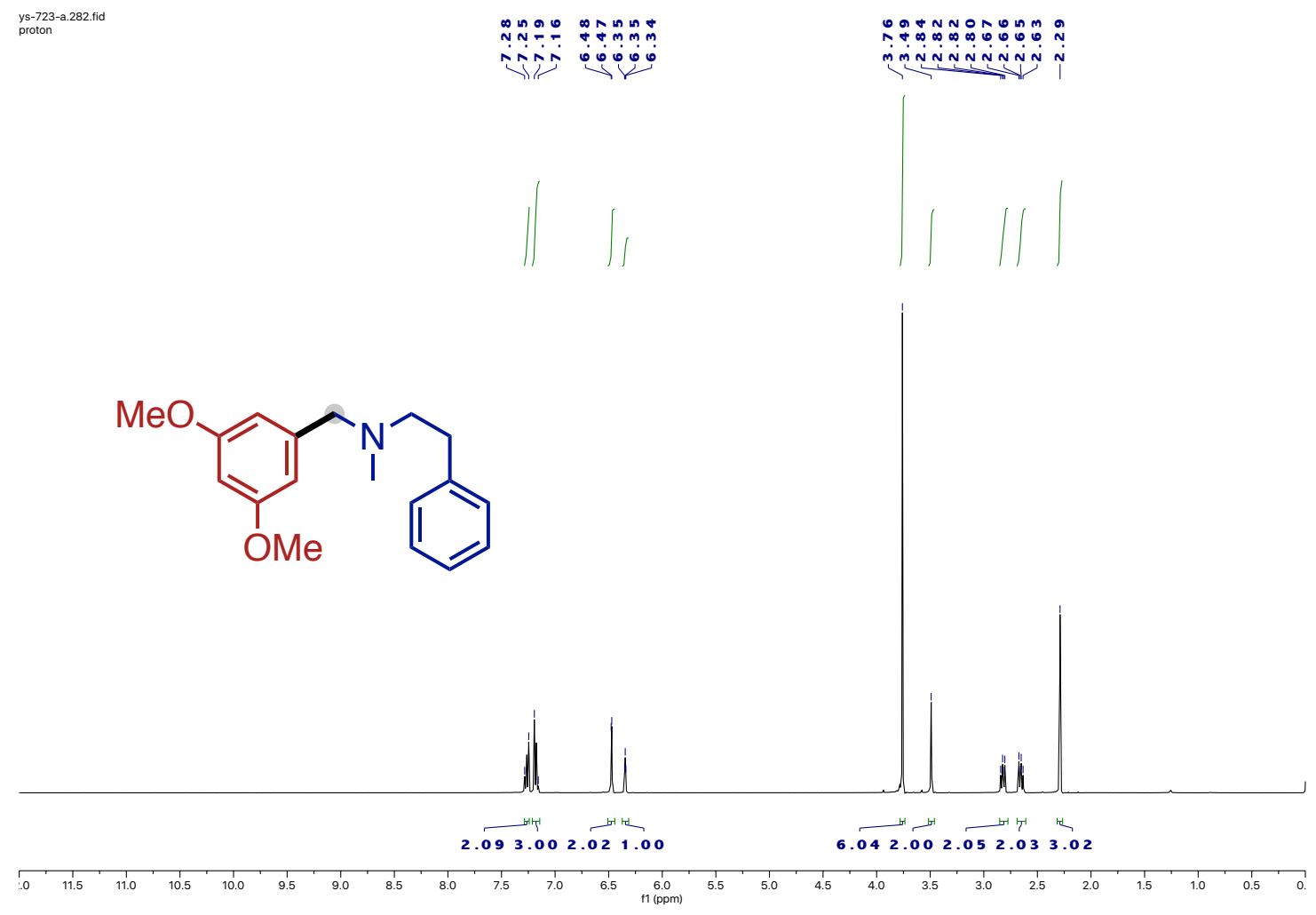

ys-723-a-c13.283.fid

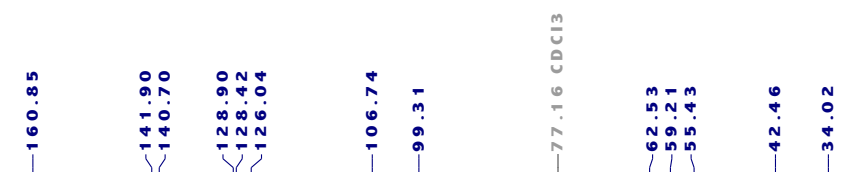
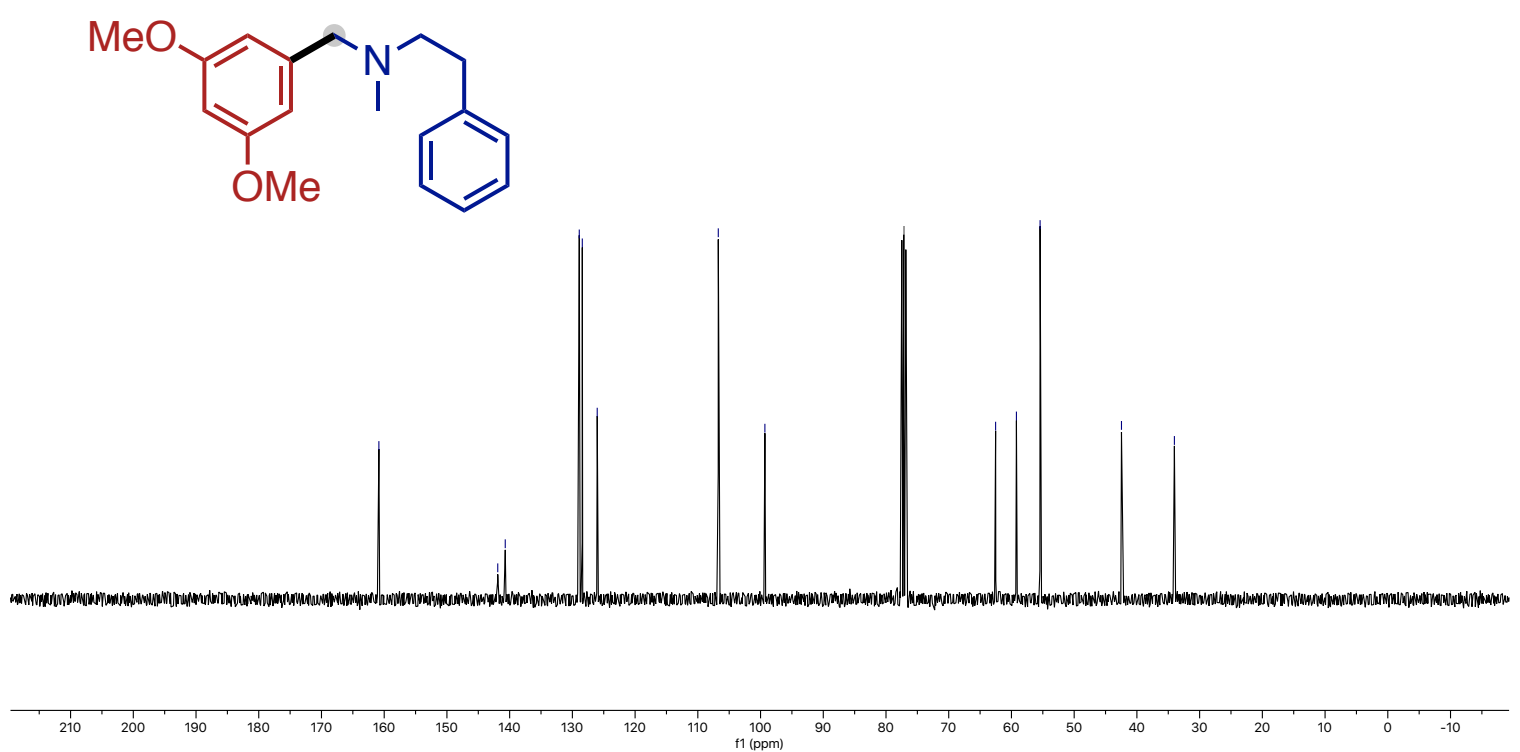


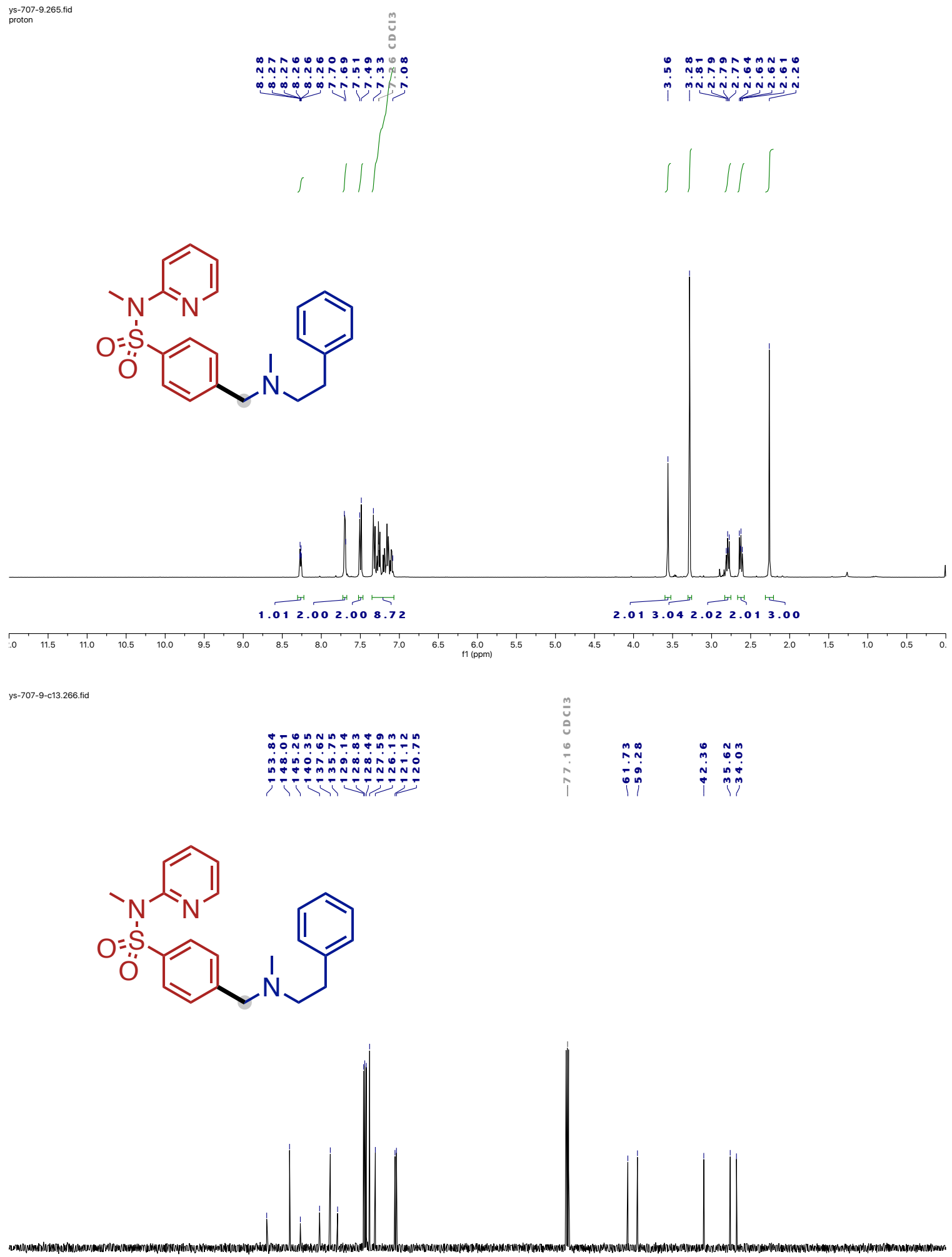

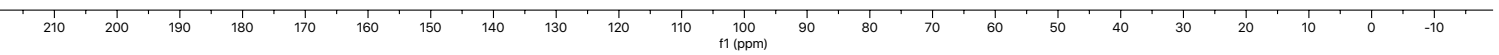



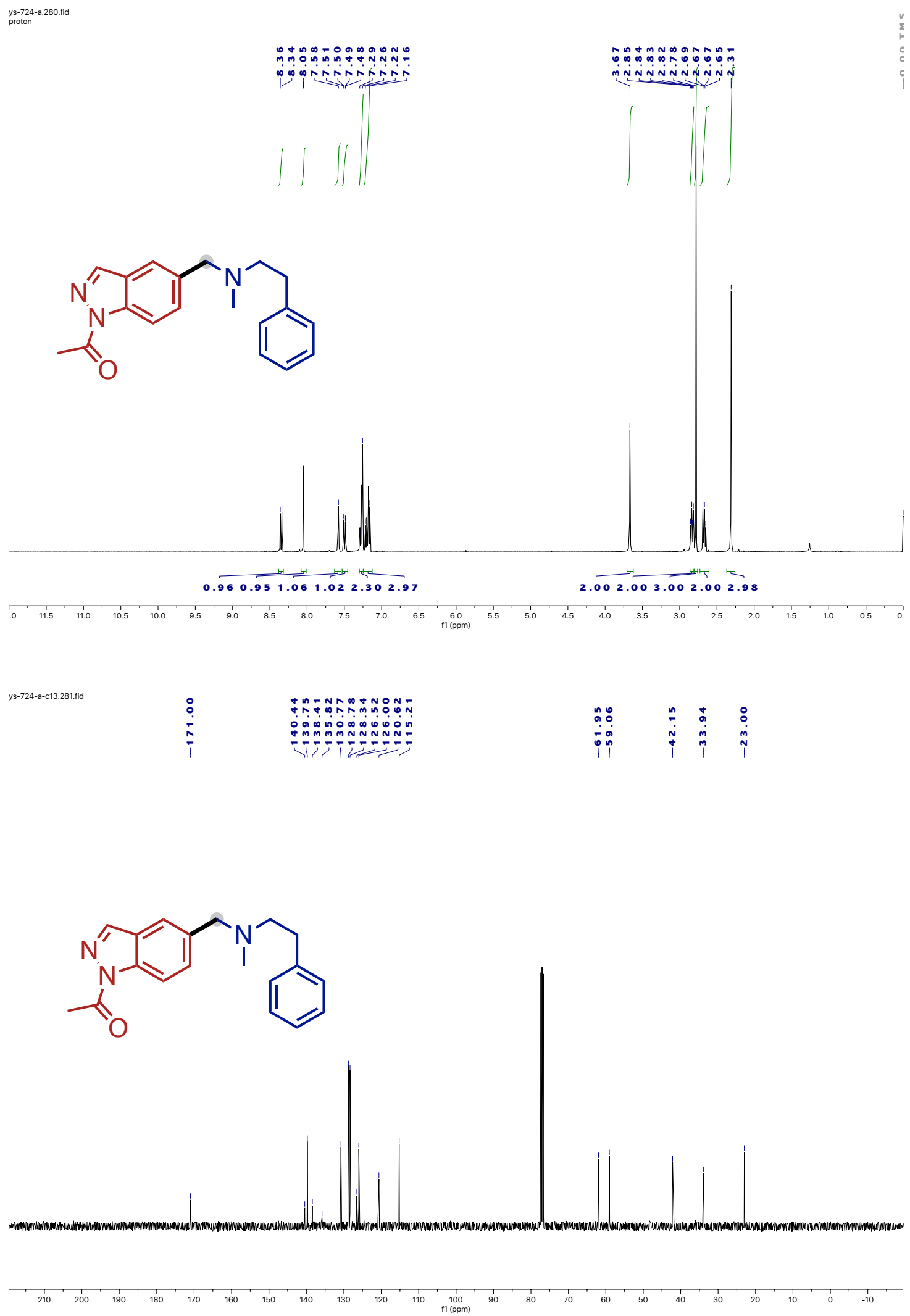
ys-722.276.fid
proton

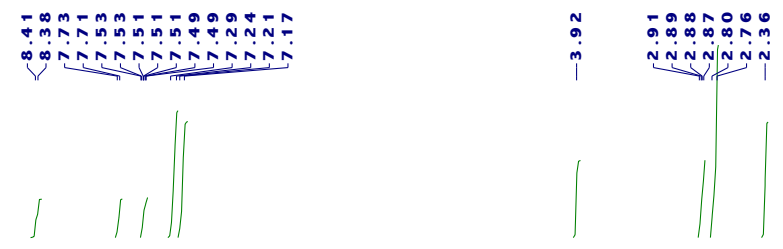<smiles>CC(=O)n1nc(CN(C)CCc2ccccc2)c2ccccc21</smiles>

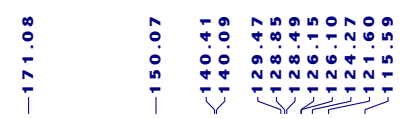<smiles>CC(=O)Nc1ccccc1C(=N)CN(C)CCc1ccccc1</smiles> 

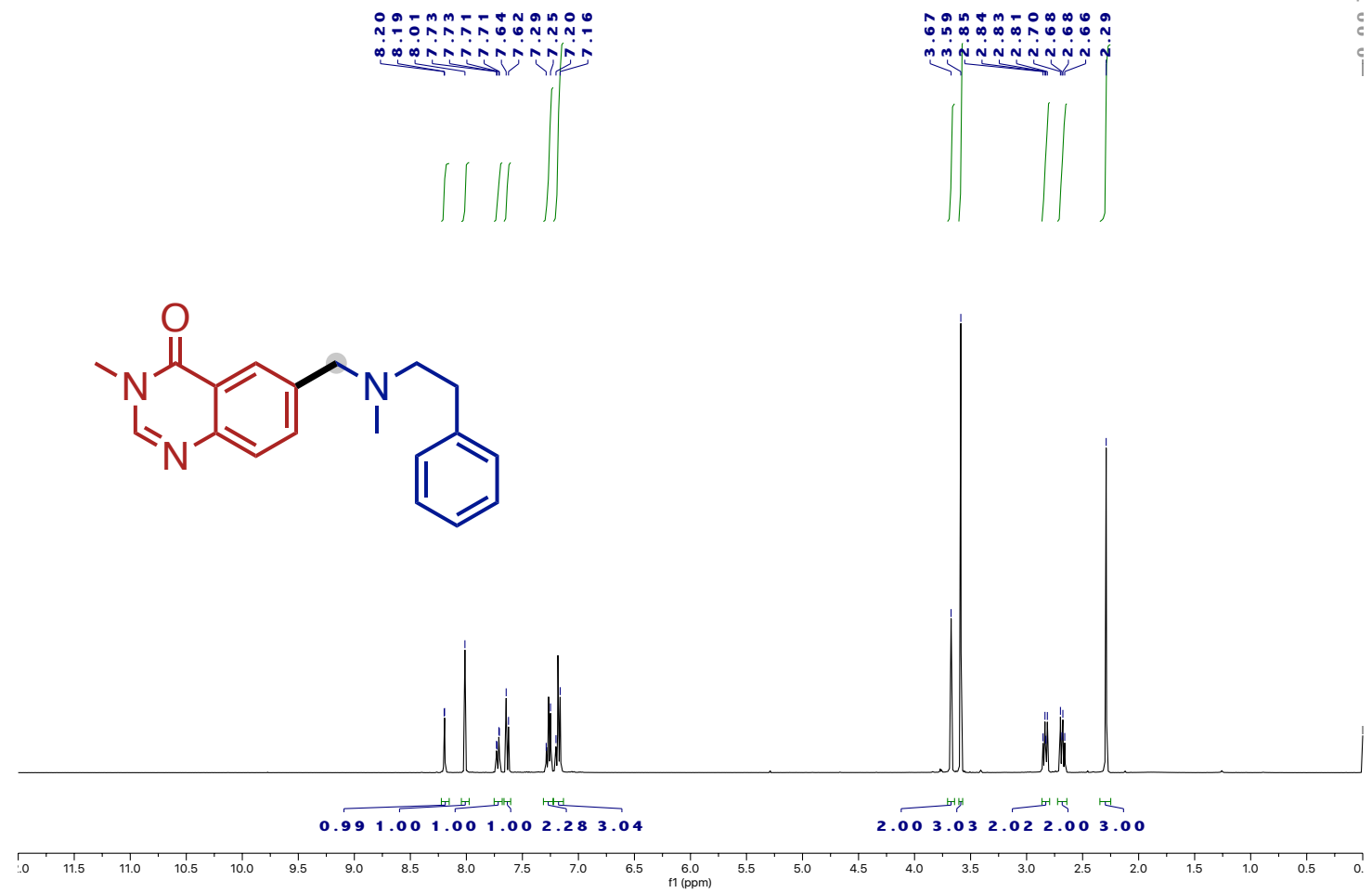

ys-718--13.366.fid

$$
\begin{array}{llll}
m & 1 \\
0 & 0
\end{array}
$$

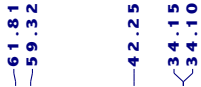<smiles>CN(CCc1ccccc1)Cc1ccc2ncn(C)c(=O)c2c1</smiles>

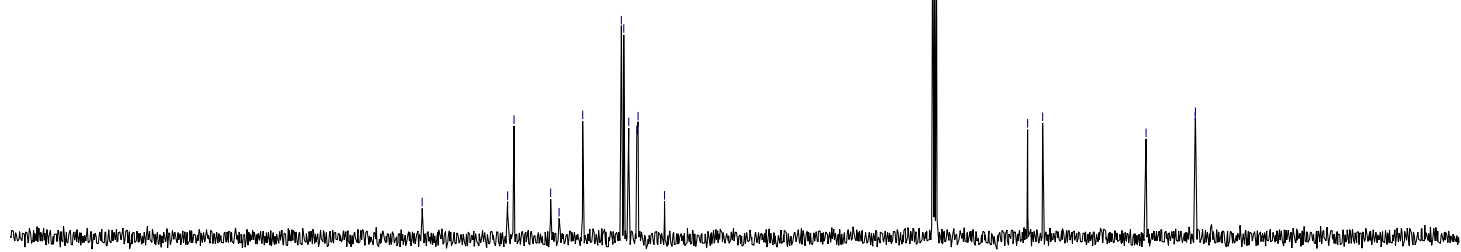

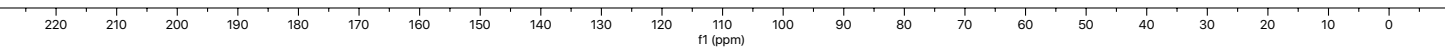




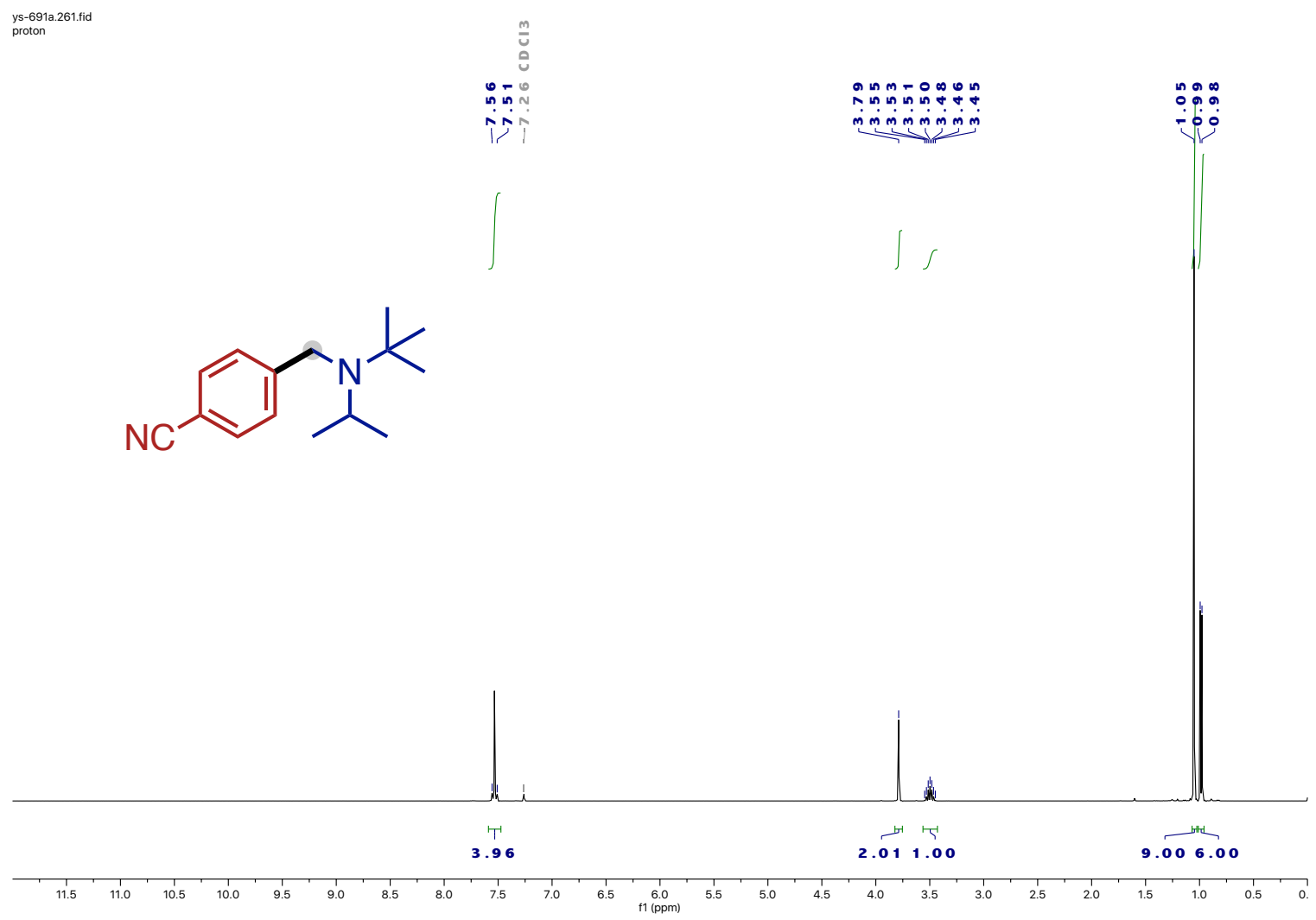

ys-691a-c13.262.fid
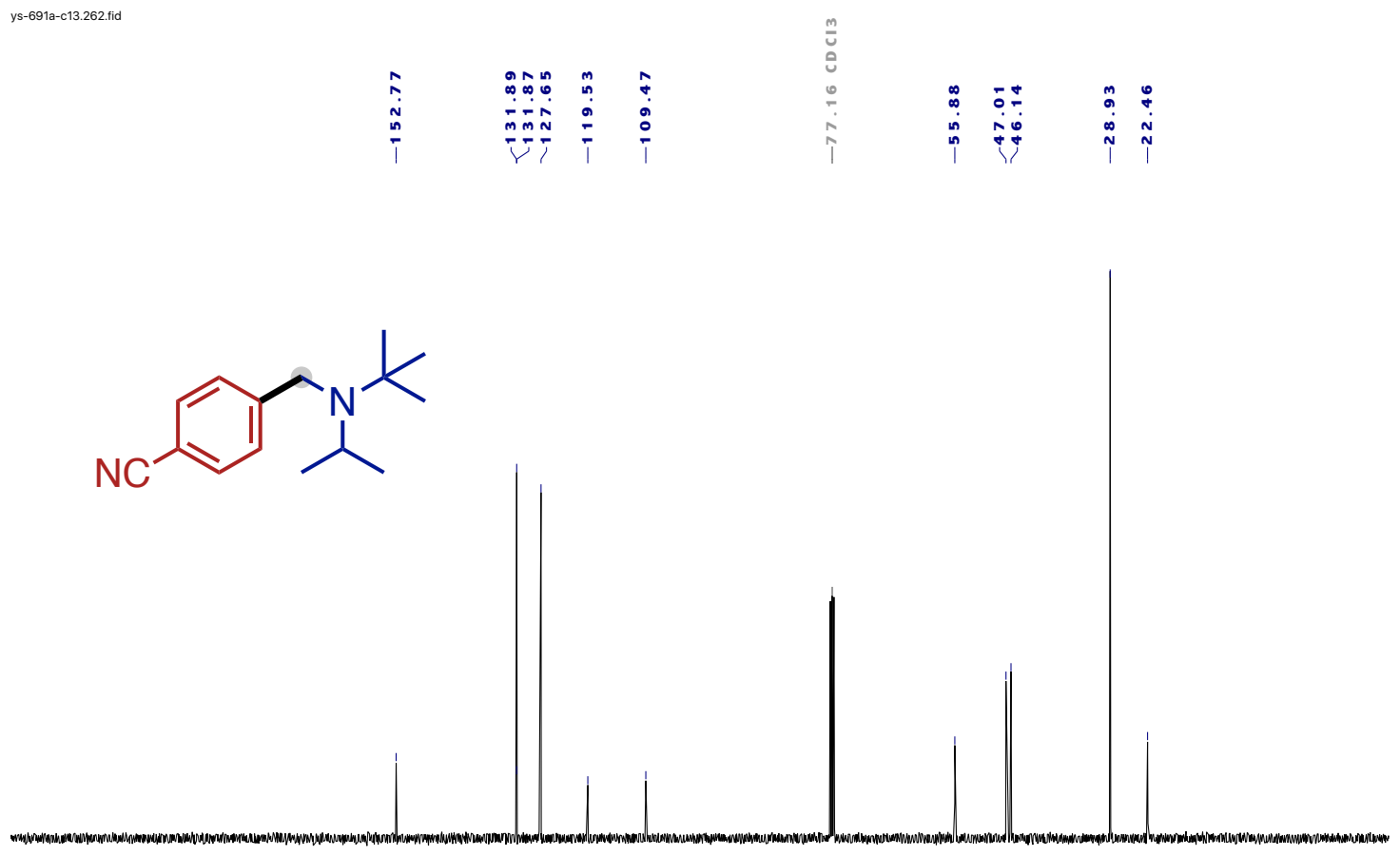


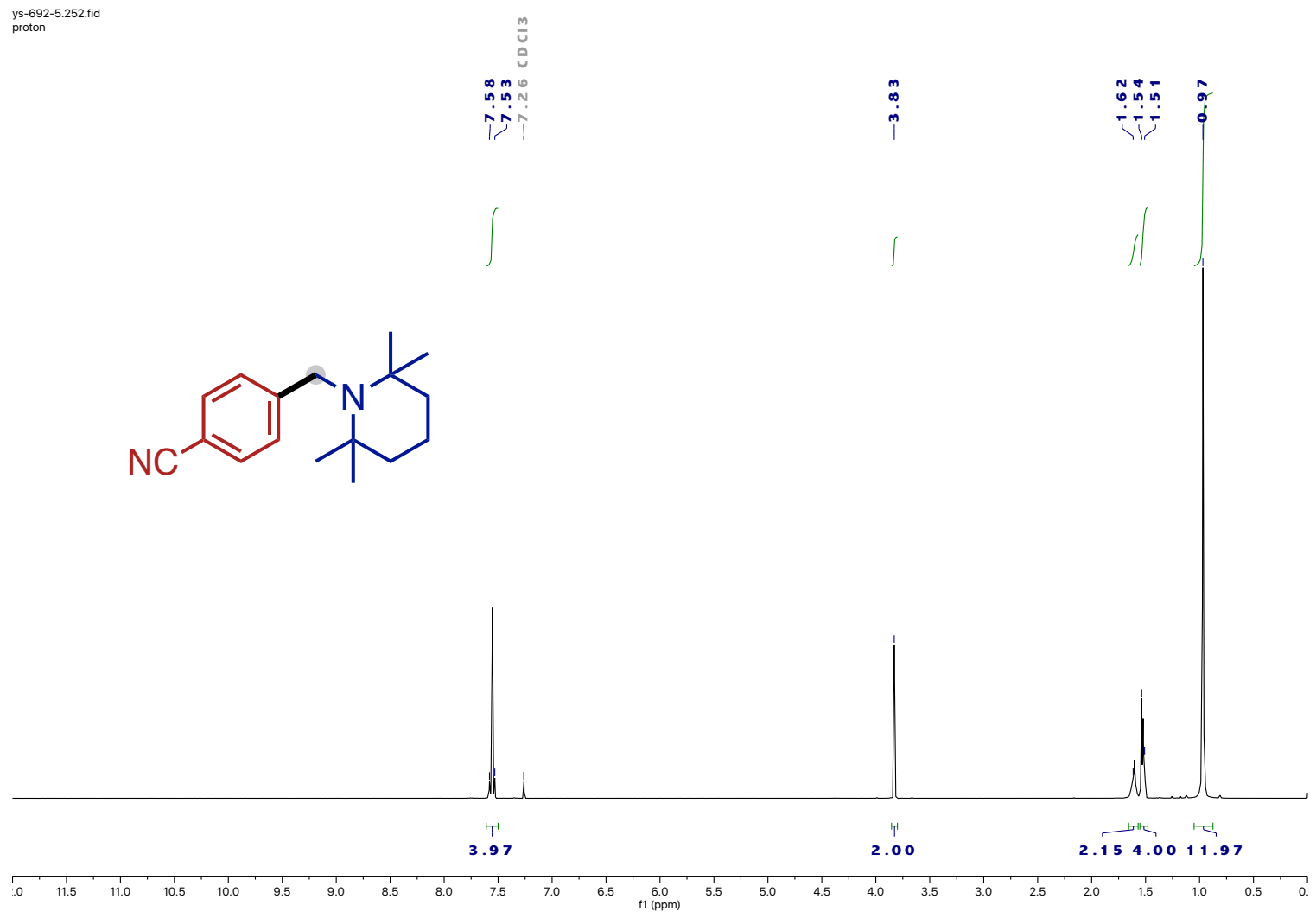

ys-692-5-c13.253.fid

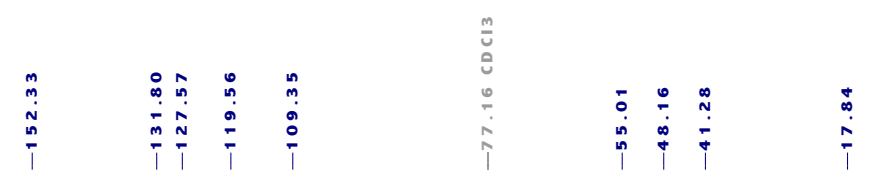<smiles>CC1(C)CCCC(C)(C)N1Cc1ccc(C#N)cc1</smiles>

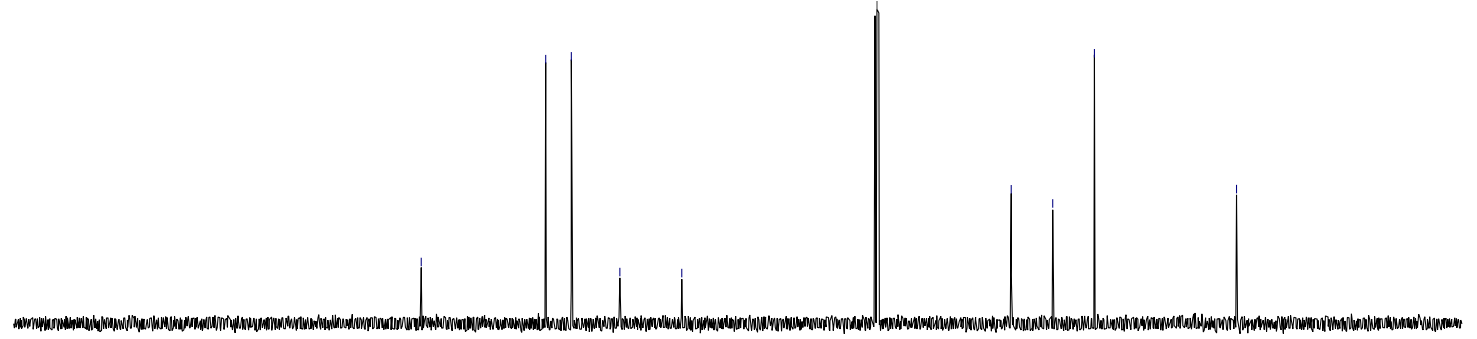




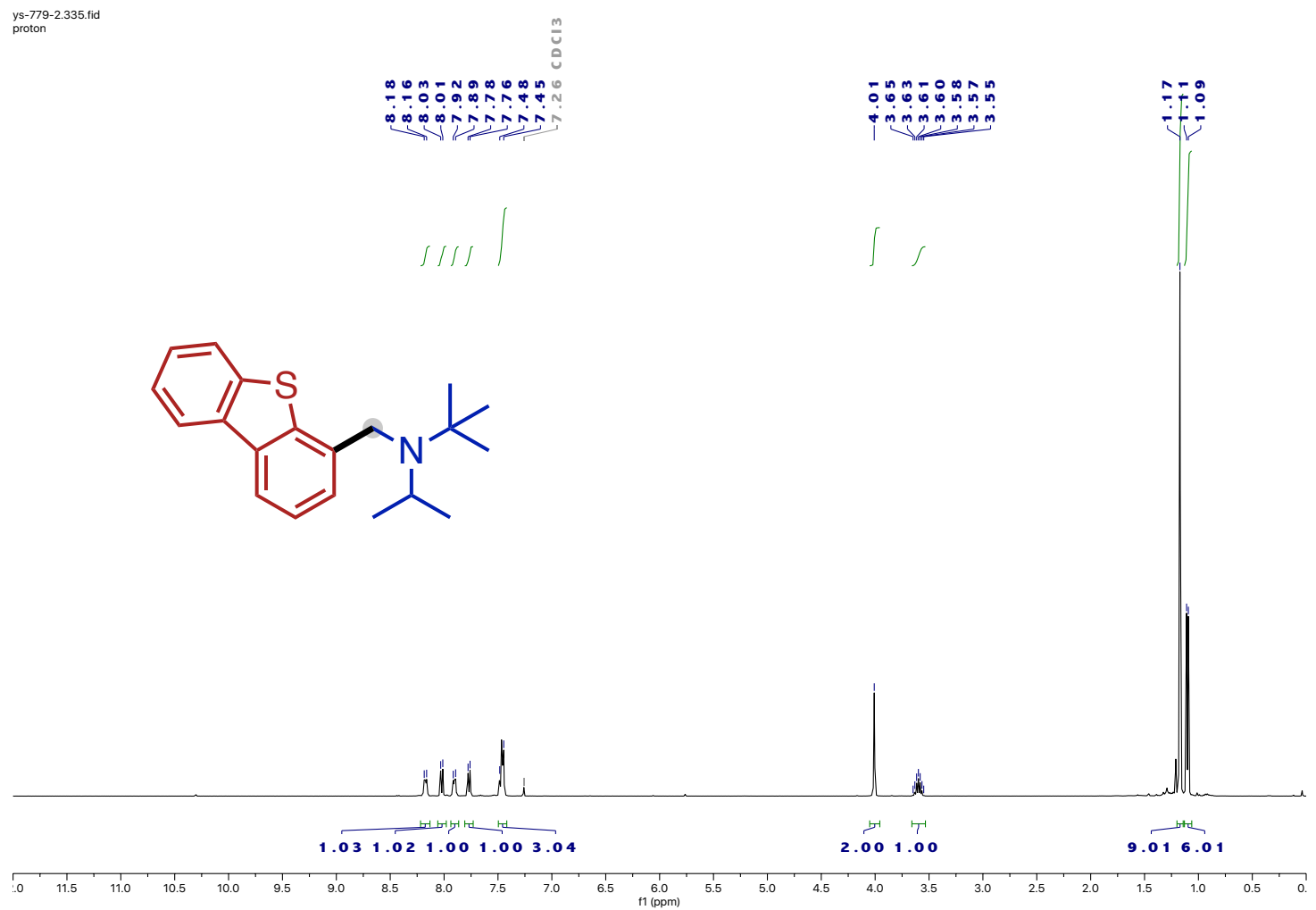

ys-779-2-a-c13.342.fid

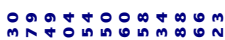

OMIOMOMONNNNNA

-

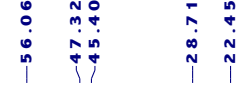<smiles>CC(C)N(Cc1cccc2c1sc1ccccc12)C(C)(C)C</smiles>

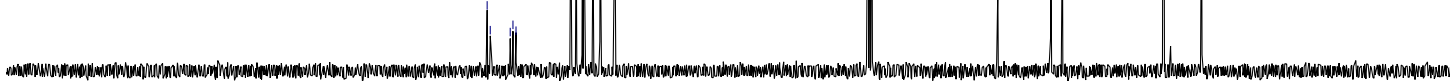

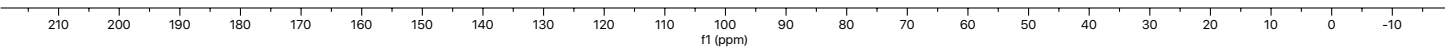



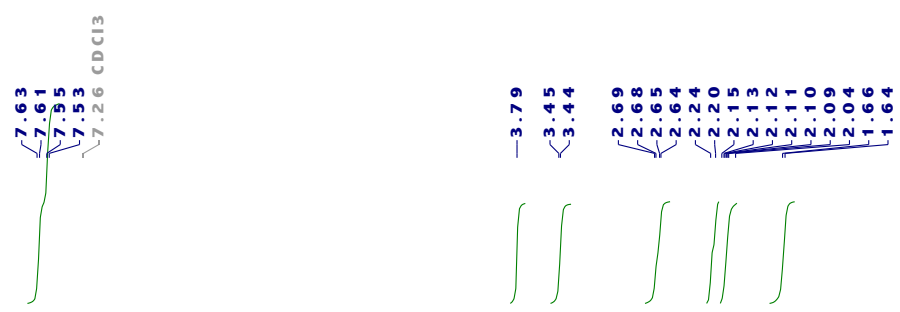

i m

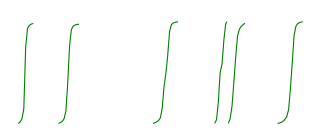

NC)

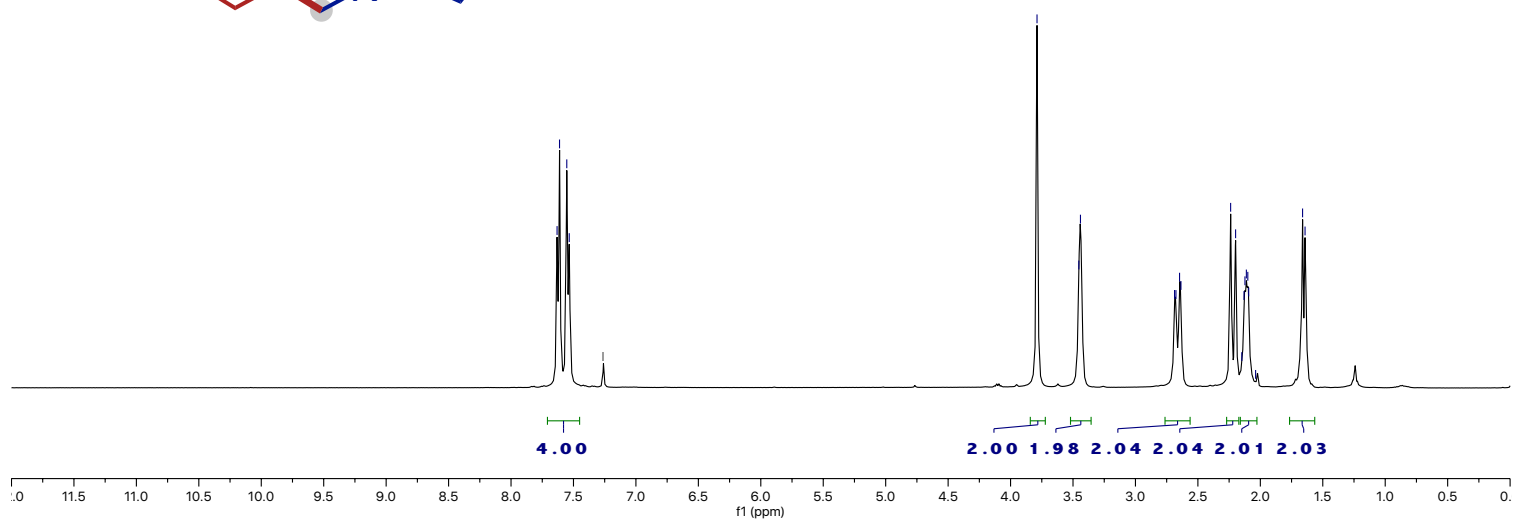

ys-714--c13-aa.270.fid

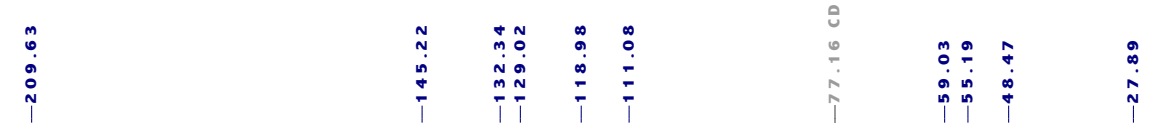

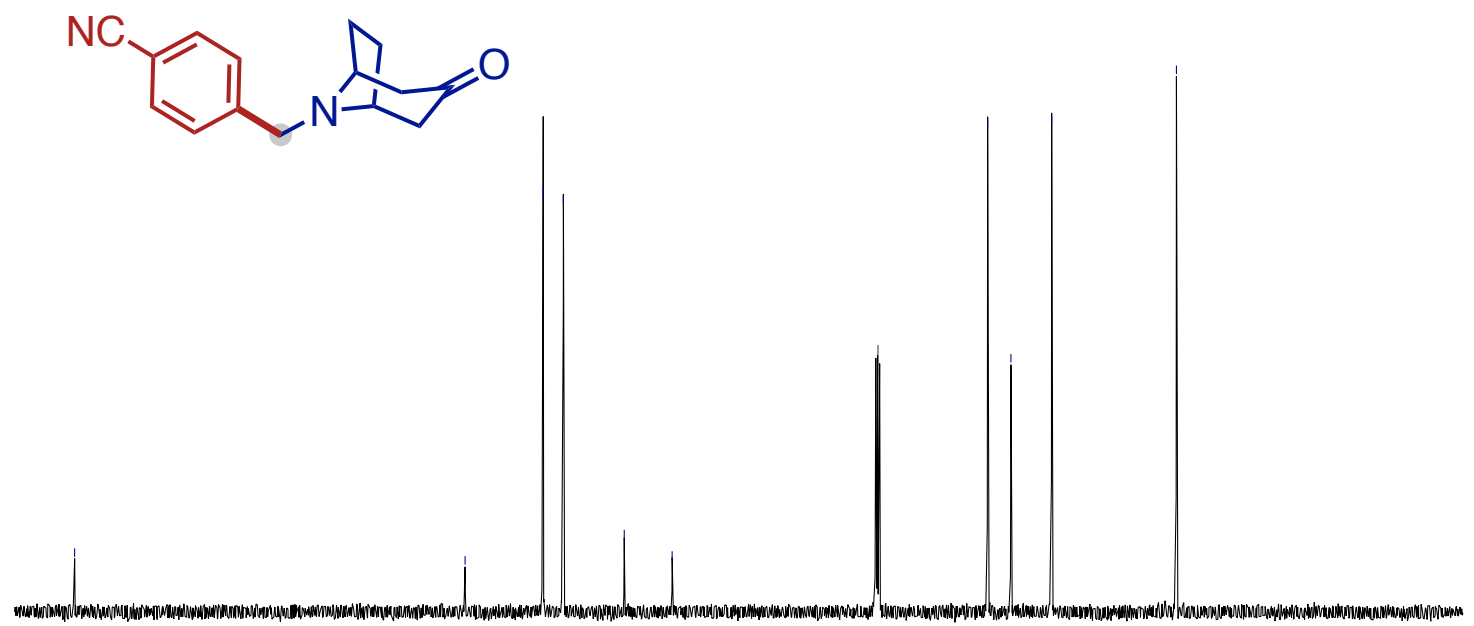



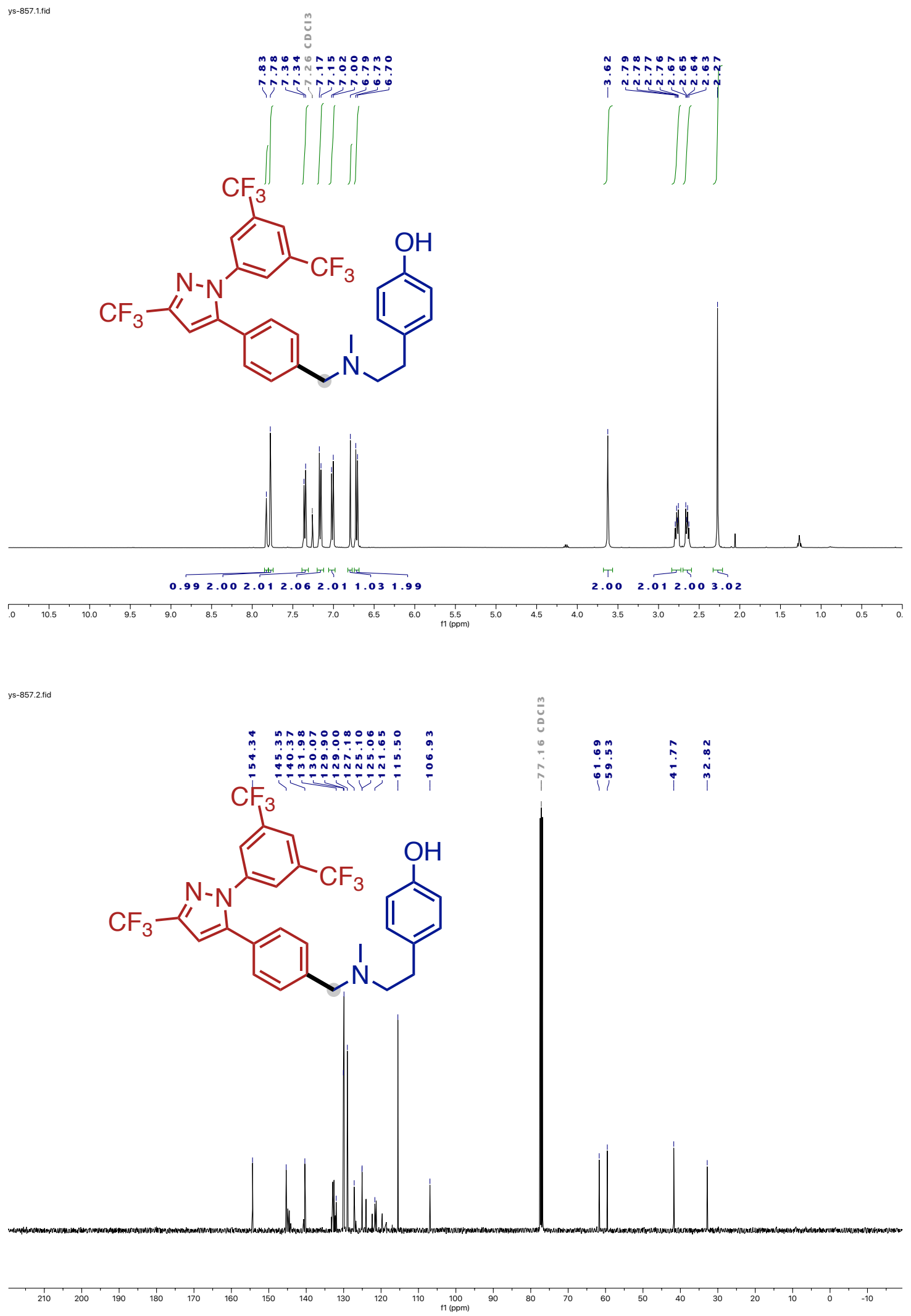

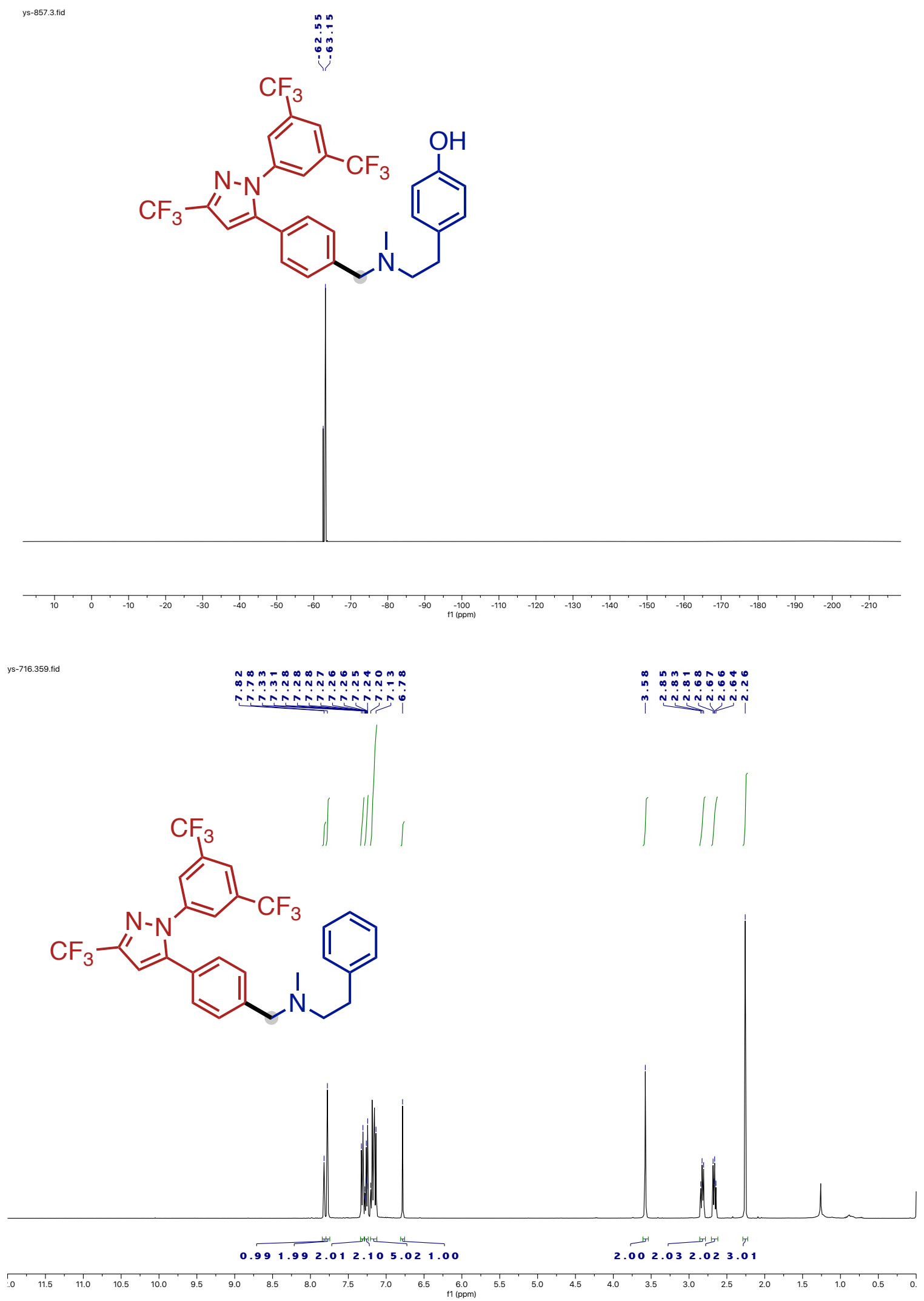
ys-716-c13.360.fid

bon 13
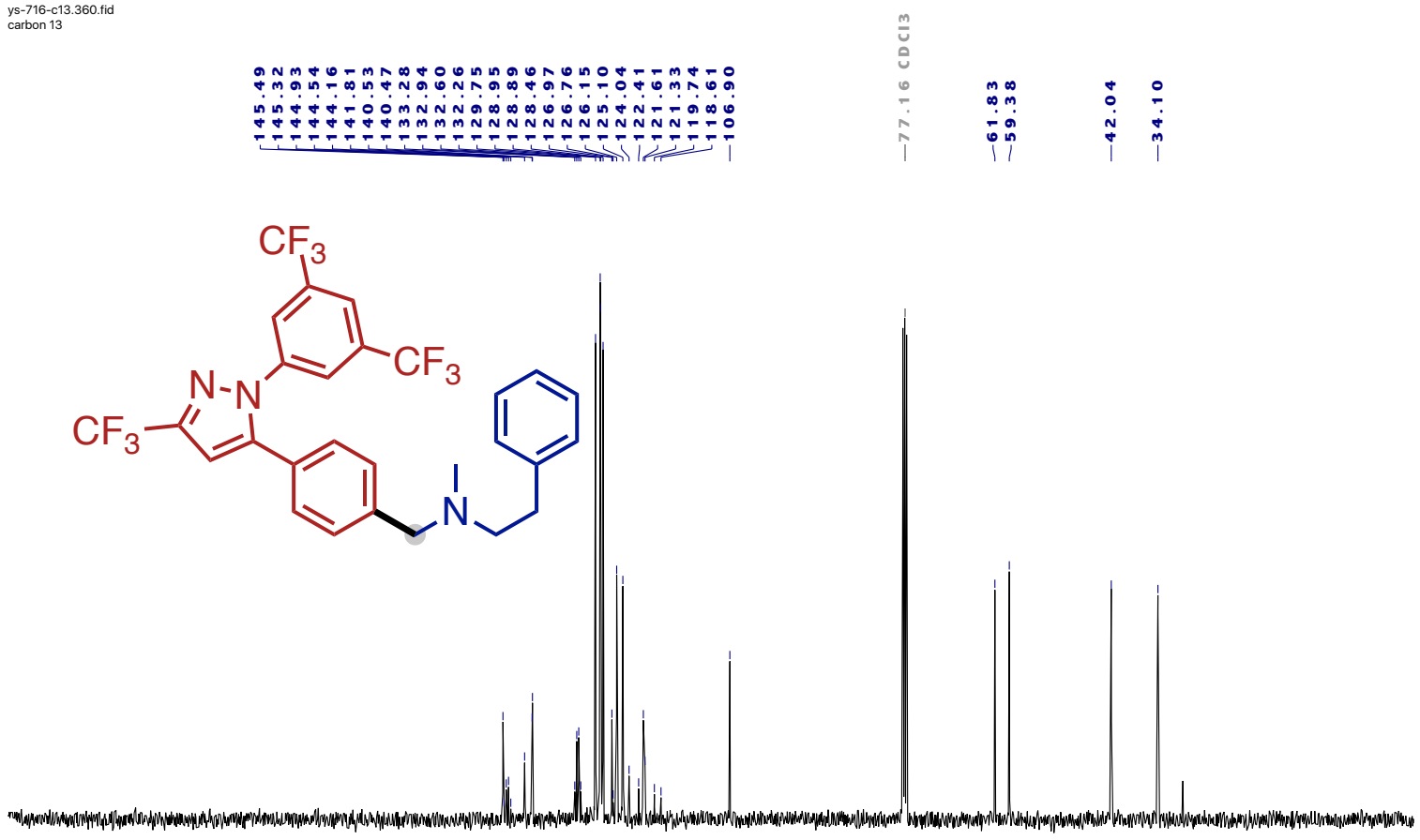

ys-716-f.271.fid
F19

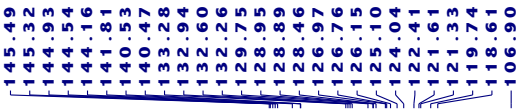

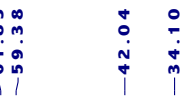

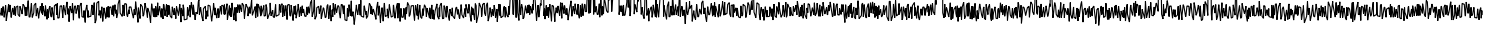

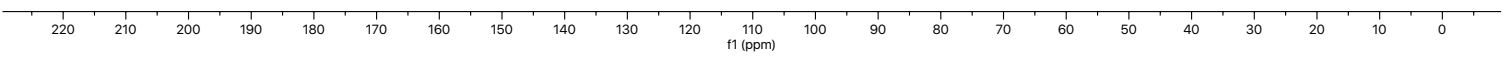<smiles>CN(CCc1ccccc1)Cc1ccc(-c2cc(C(F)(F)F)nn2-c2cc(C(F)(F)F)cc(C(F)(F)F)c2)cc1</smiles>
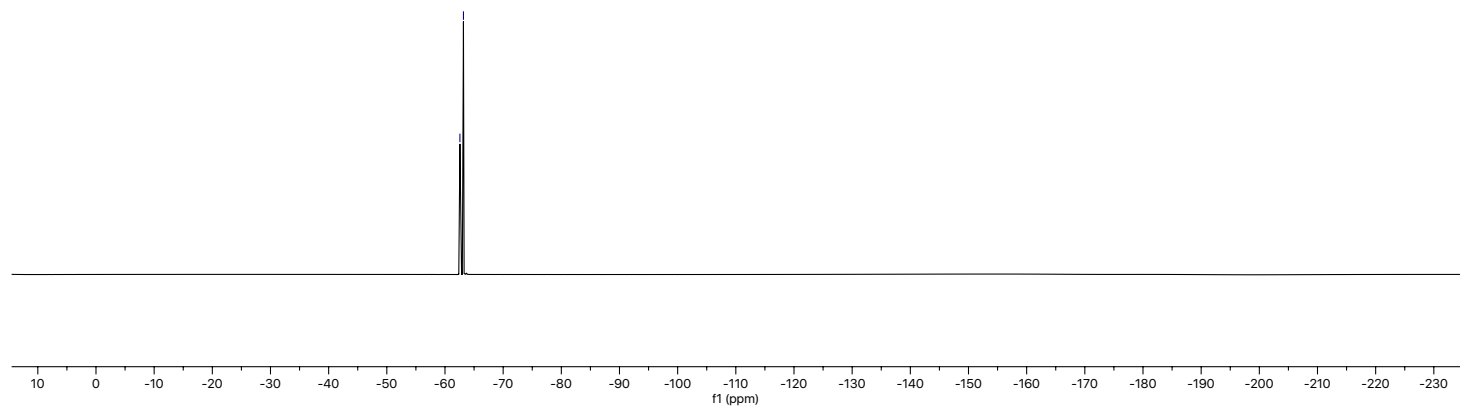

S45 


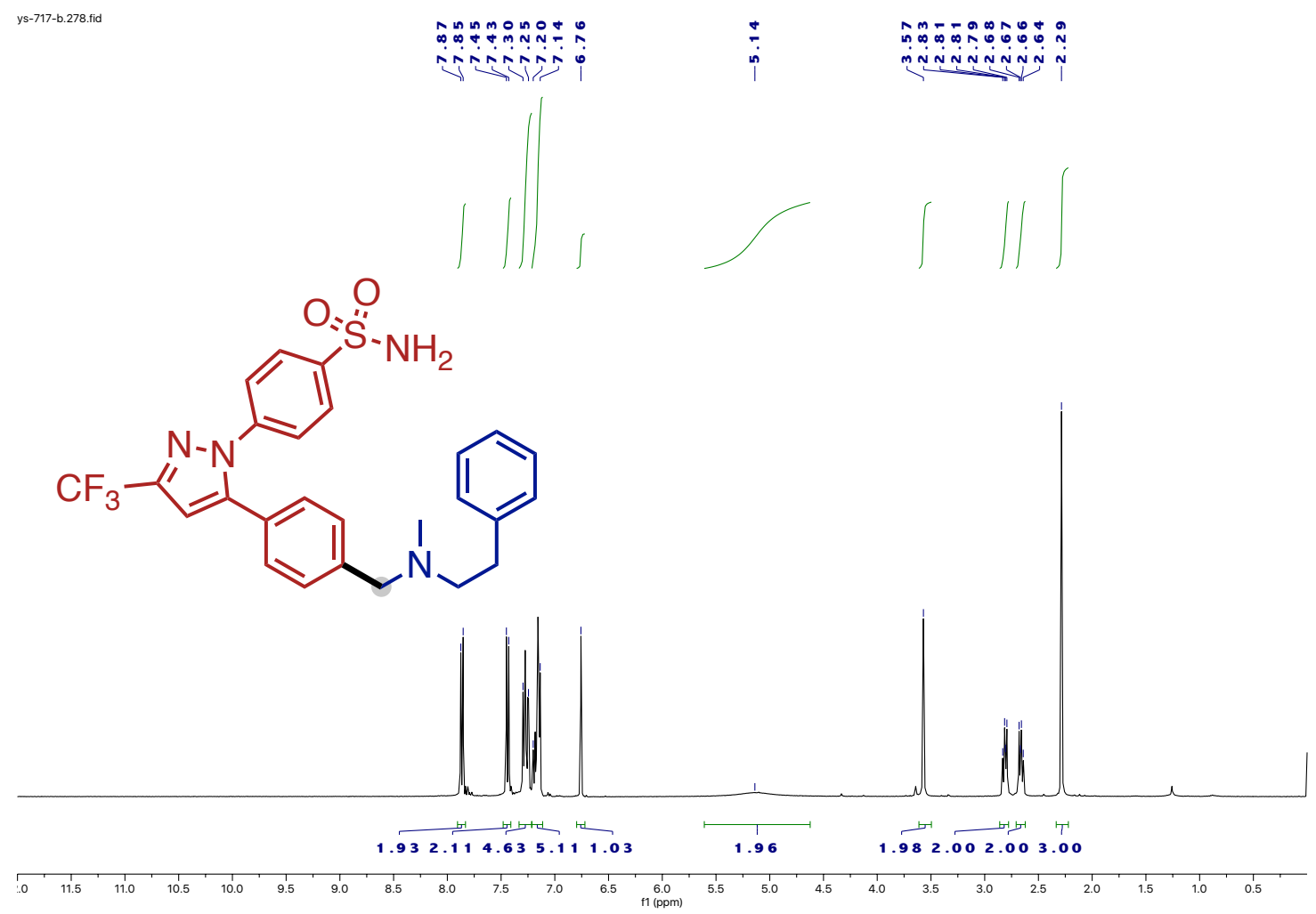

ys-717-b-c13.279.fid
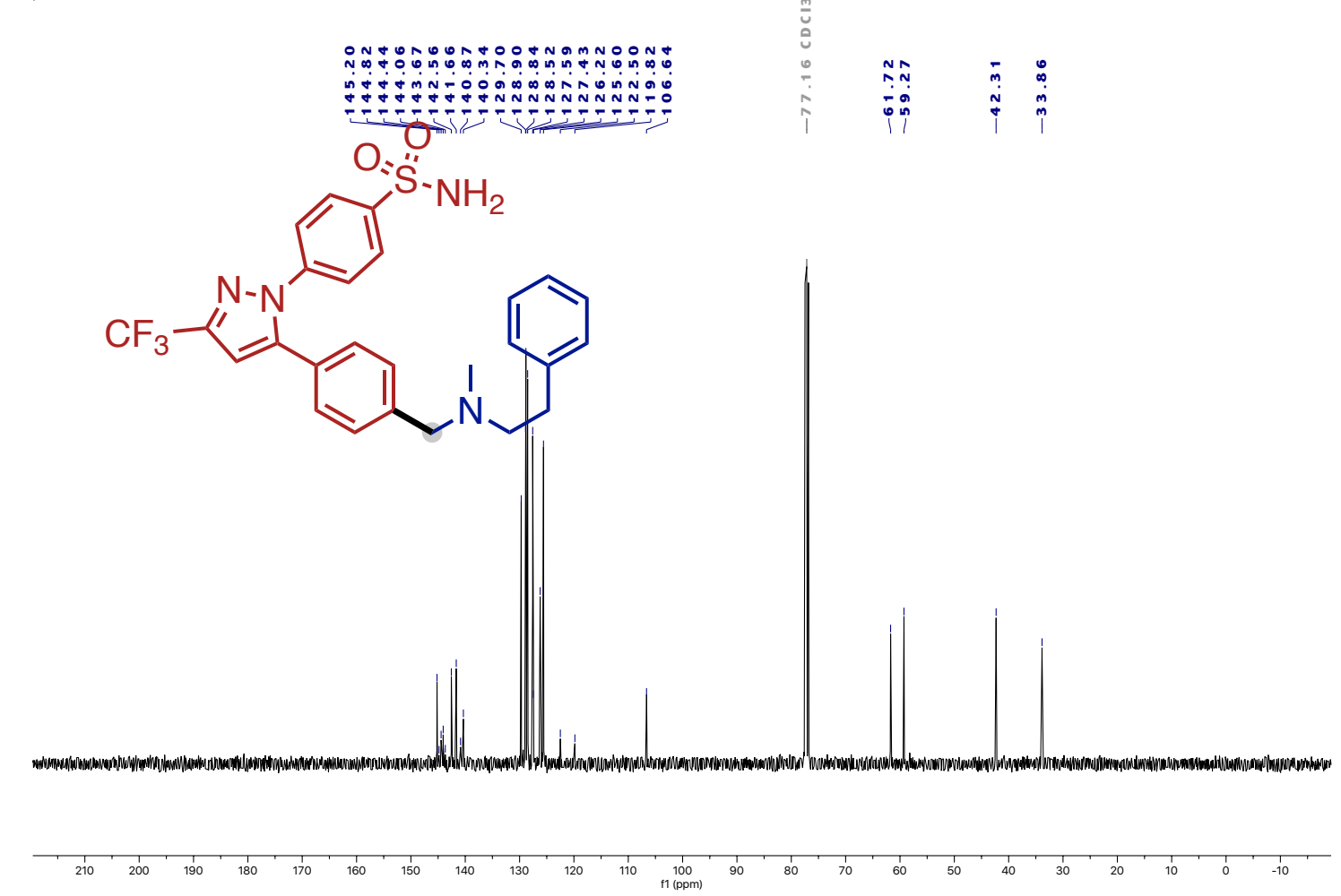

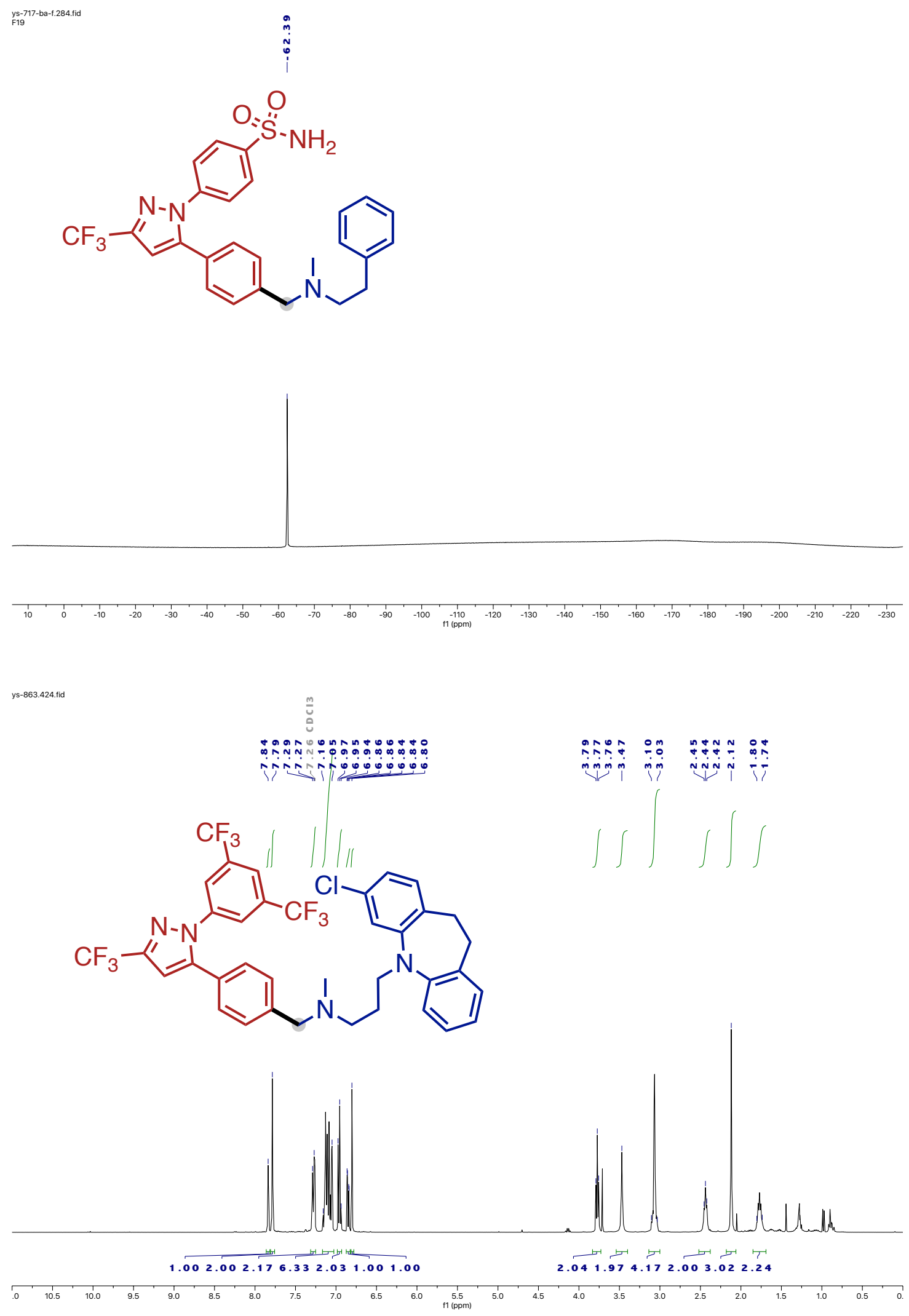

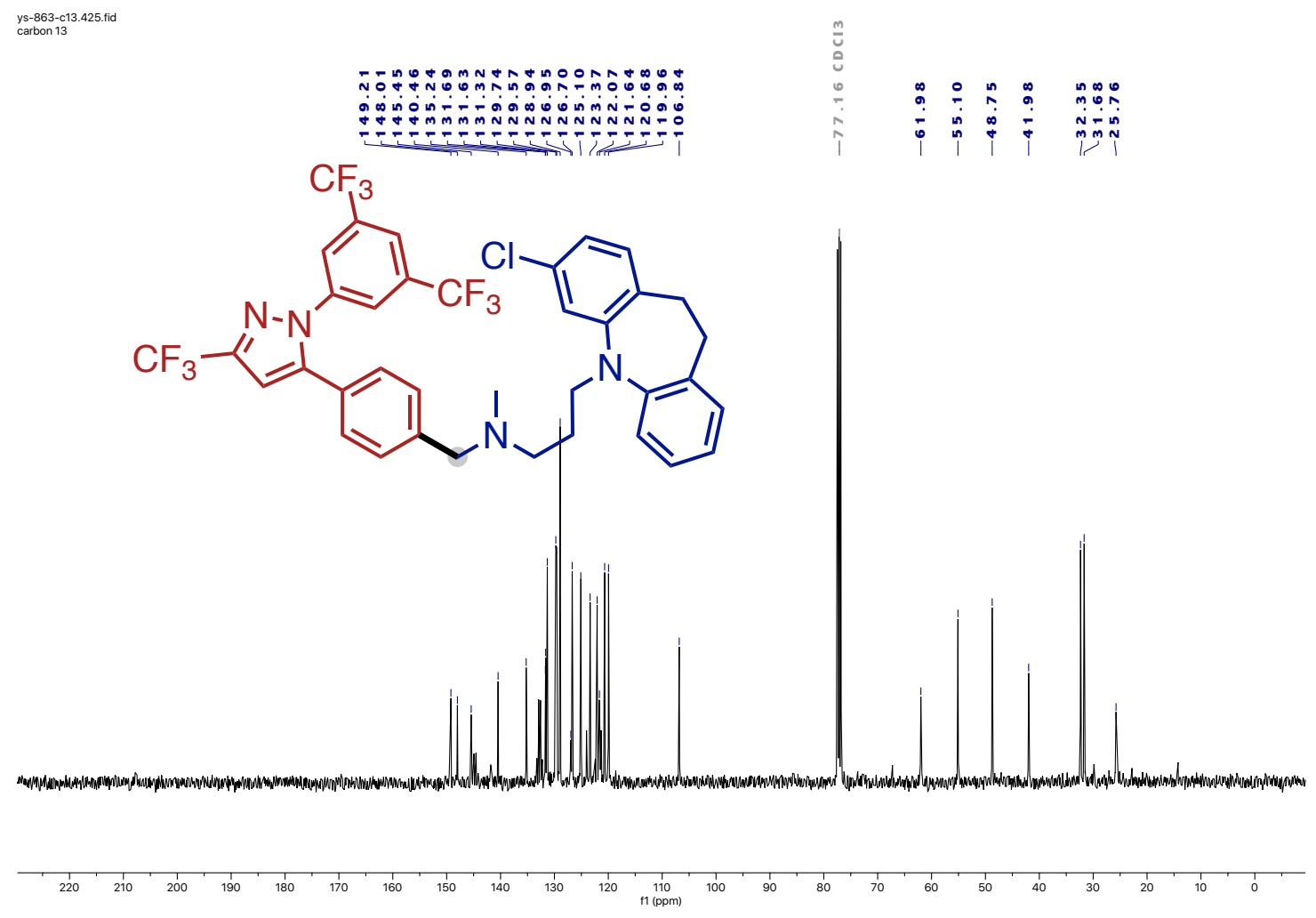

ys-863-f.389.fid
F19

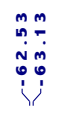<smiles>CN(CCCN1c2ccccc2CCc2ccc(Cl)cc21)Cc1ccc(-c2cc(C(F)(F)F)nn2-c2cc(C(F)(F)F)cc(C(F)(F)F)c2)cc1</smiles>

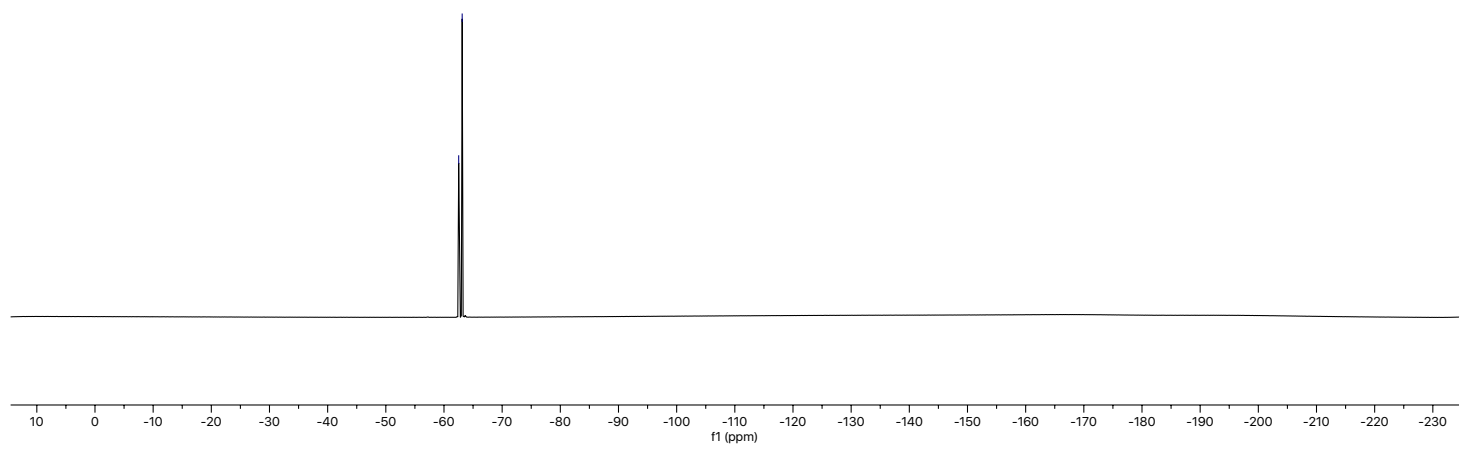



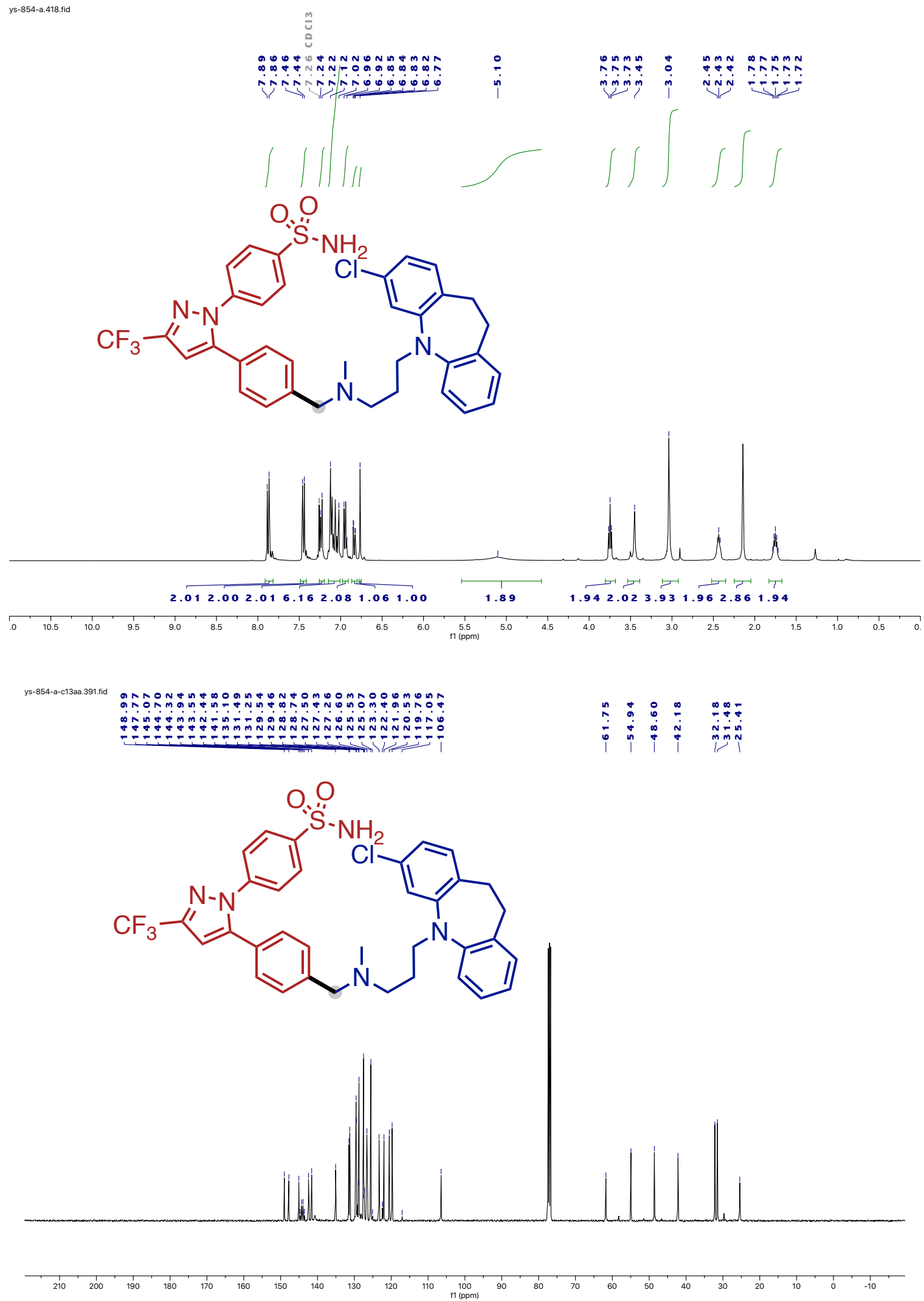

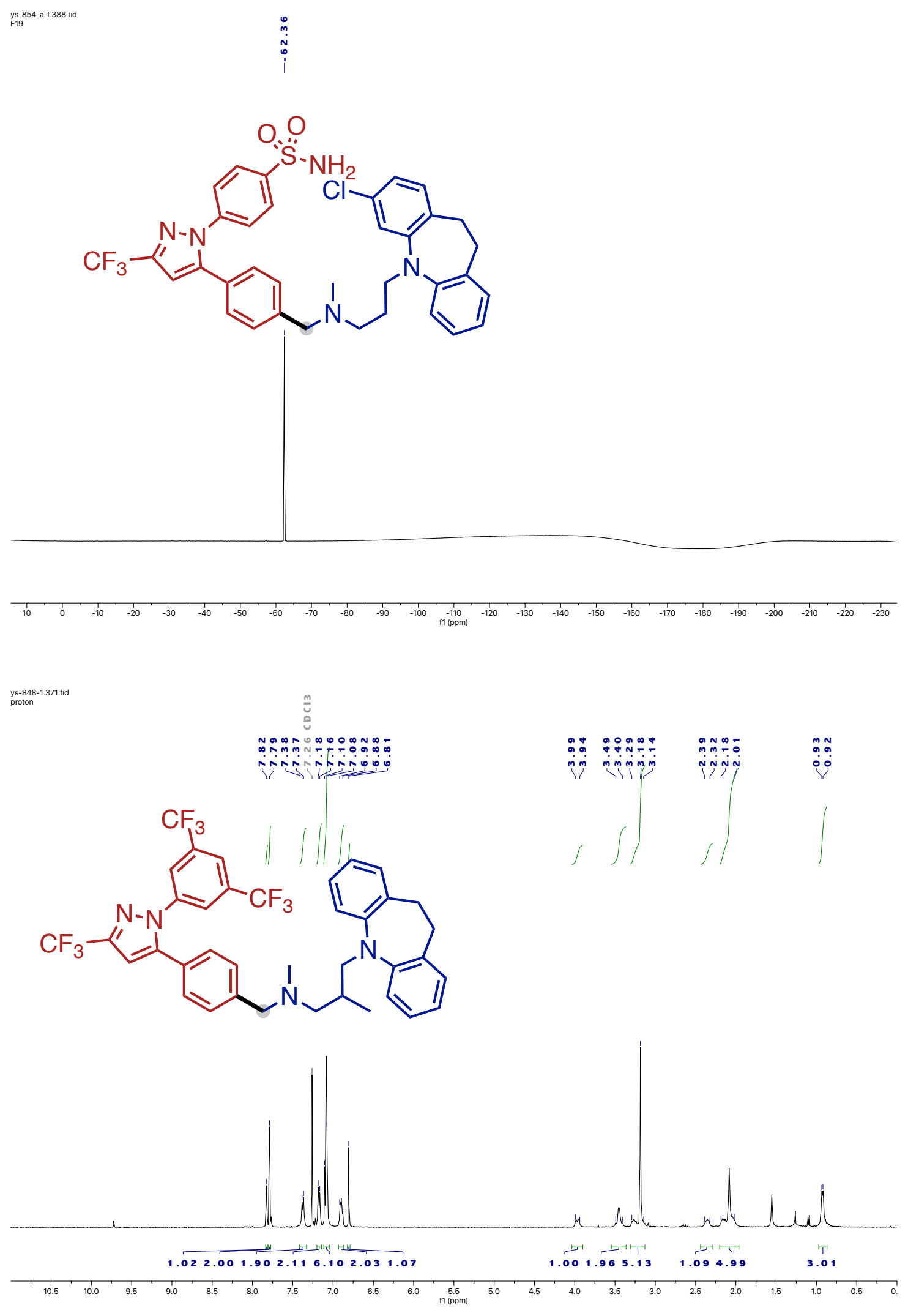

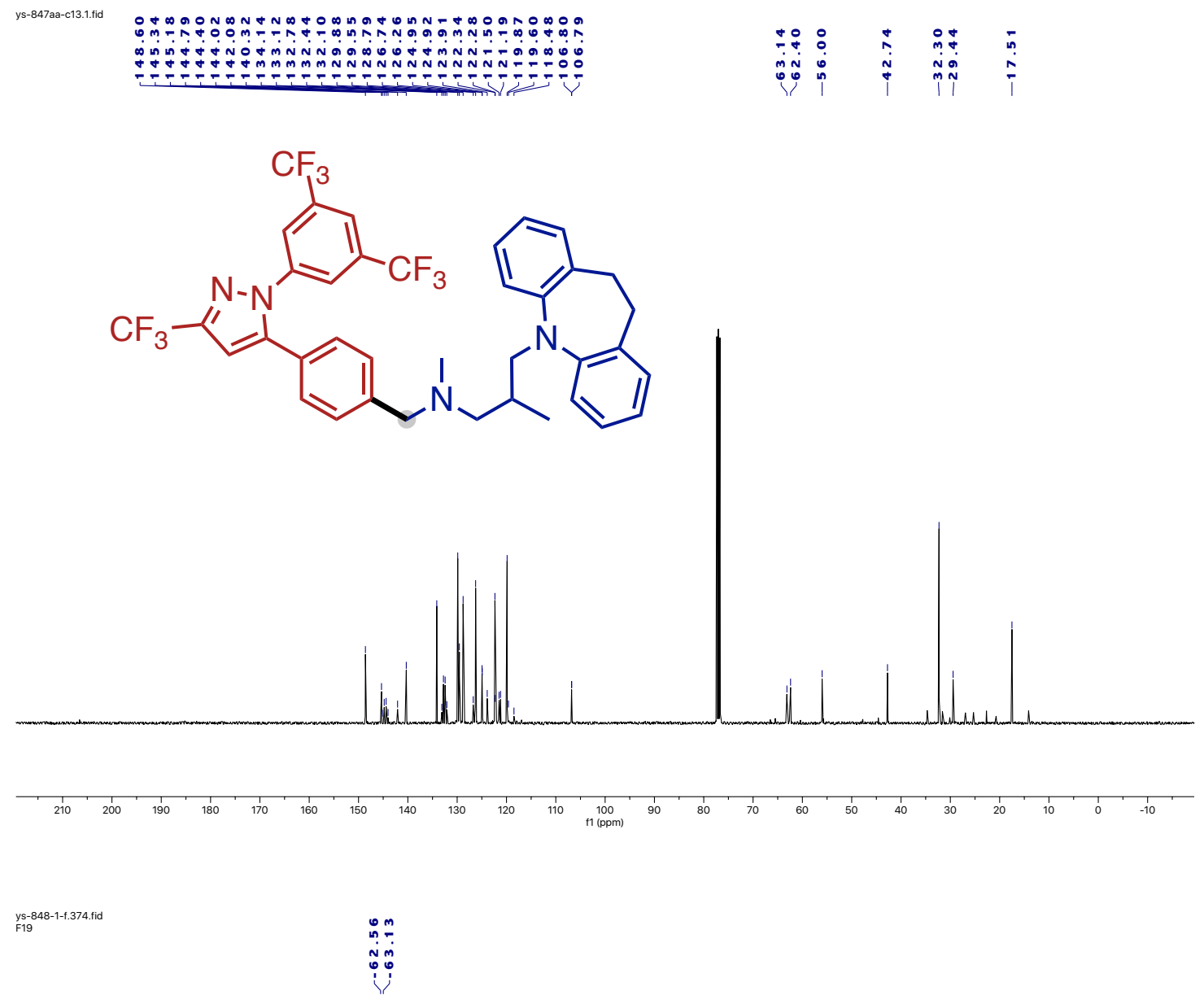<smiles>CC(CN(C)Cc1ccc(-c2cc(C(F)(F)F)nn2-c2cc(C(F)(F)F)cc(C(F)(F)F)c2)cc1)CN1c2ccccc2CCc2ccccc21</smiles>

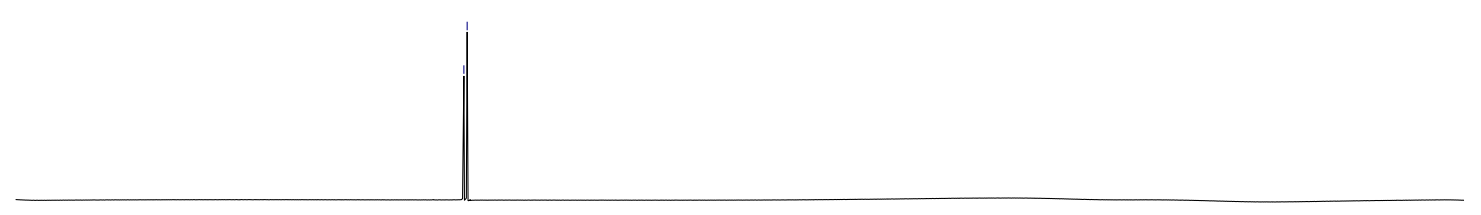

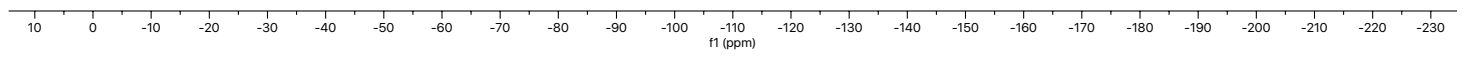



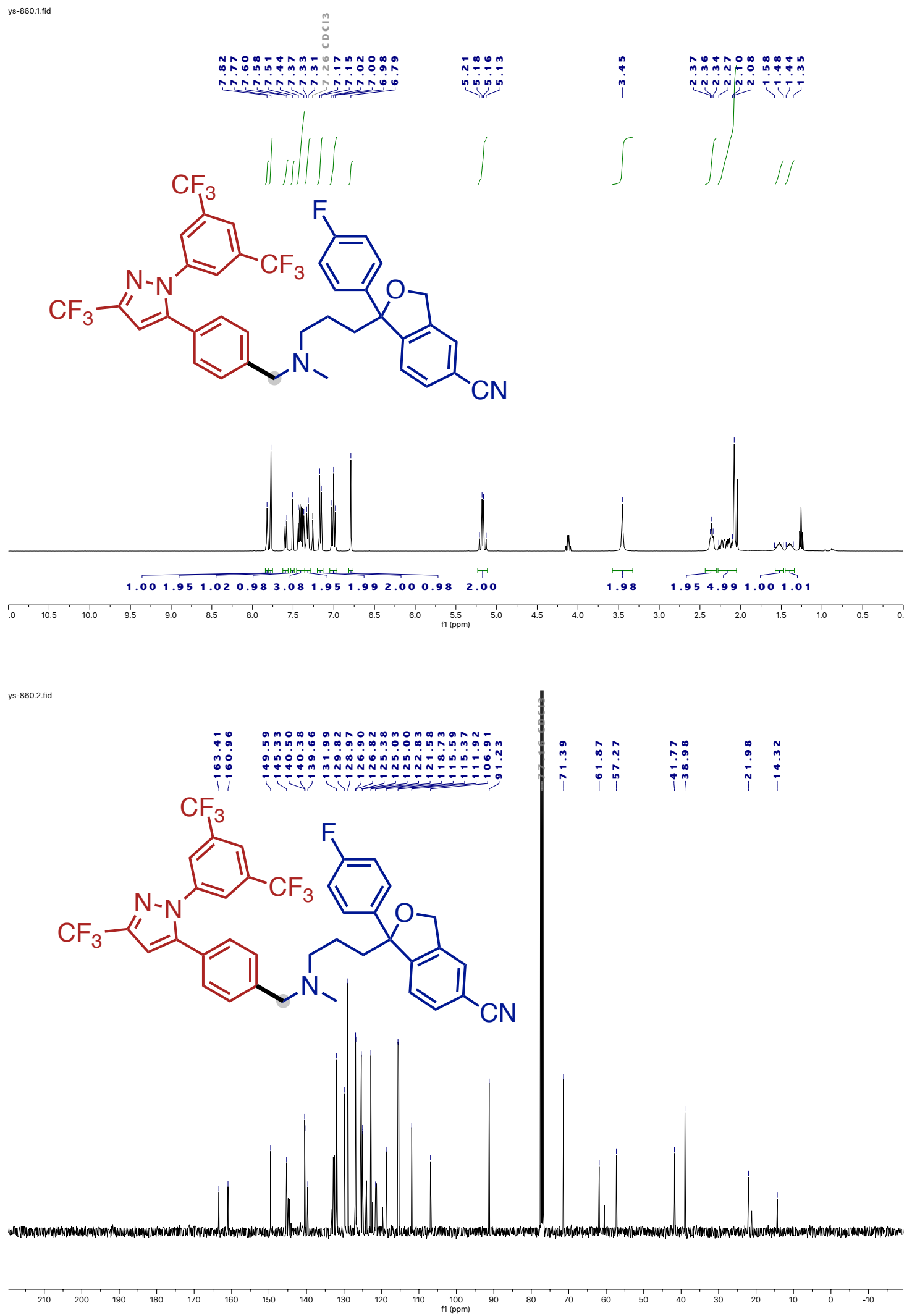

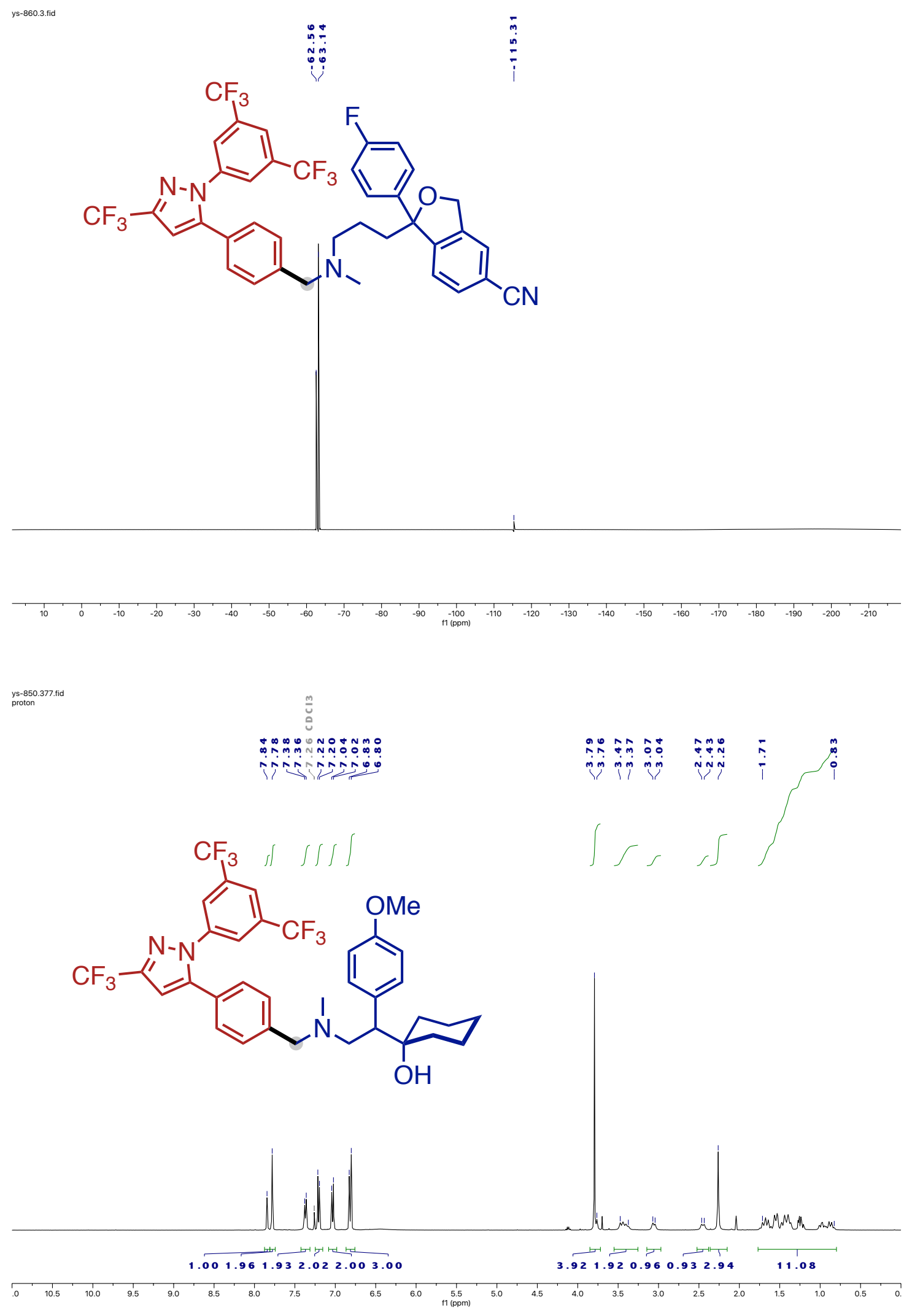

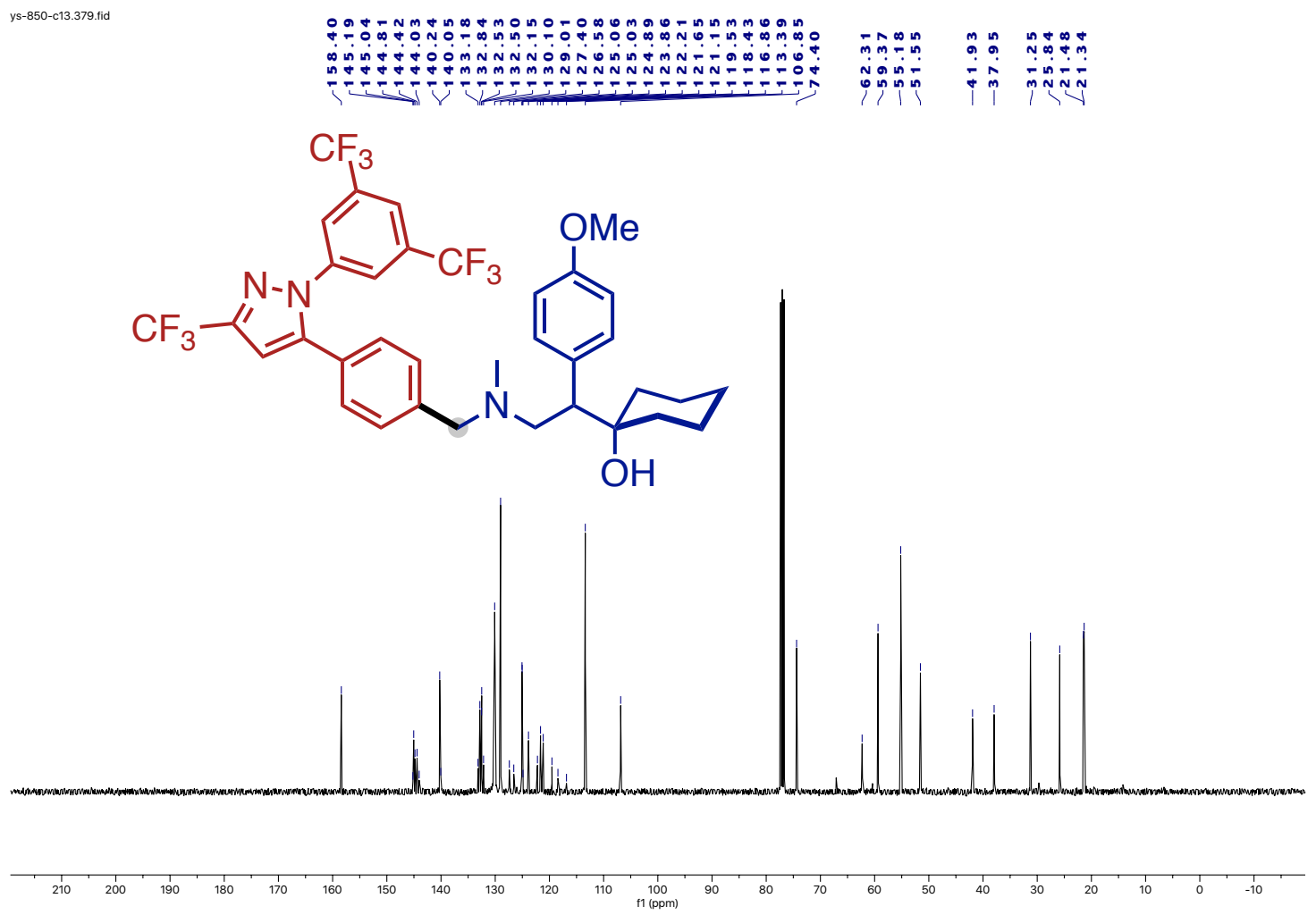

ys-850-f.378.fid
F19

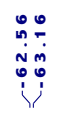

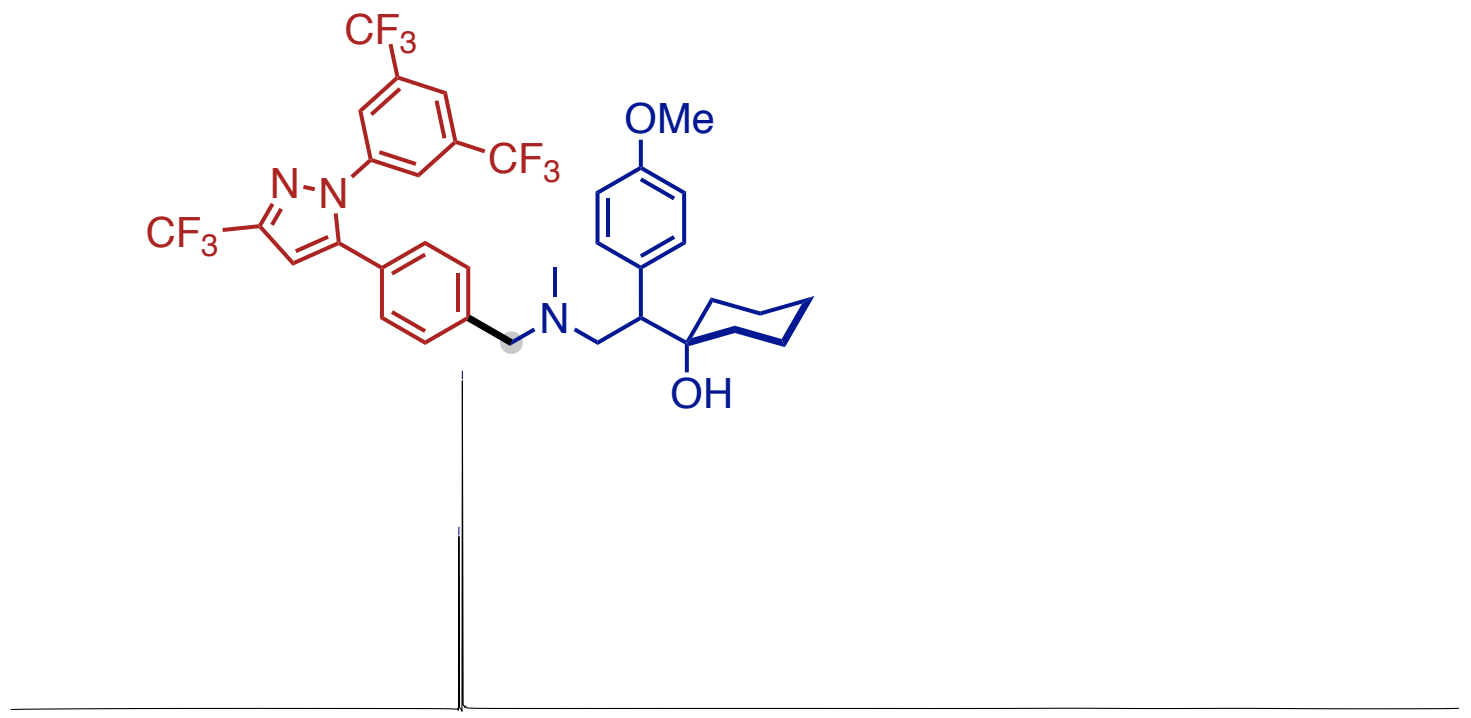

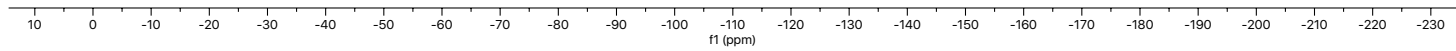




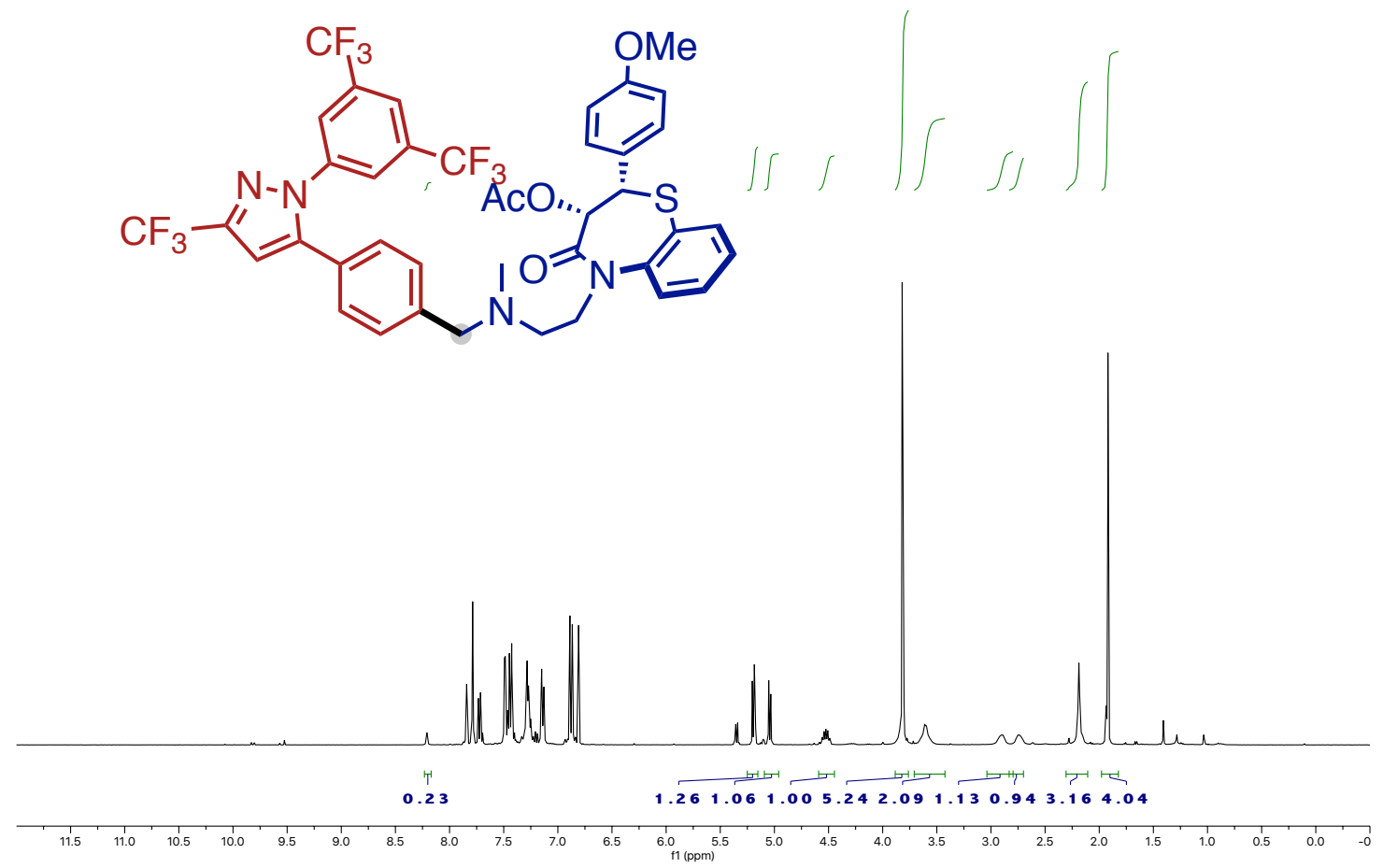

ys-848aa-c13.1.fid

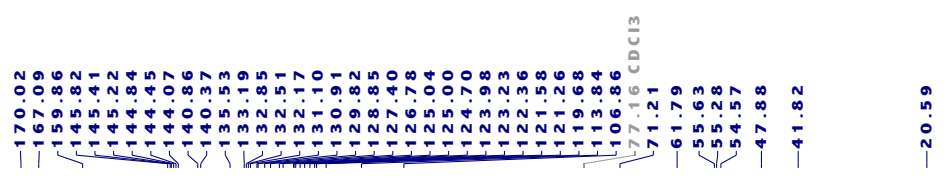<smiles>COc1ccc([C@H](Sc2ccccc2N(CCN(C)Cc2ccc(-c3cc(C(F)(F)F)nn3-c3cc(C(F)(F)F)cc(C(F)(F)F)c3)cc2)C(=O)[C@H](OC(C)(F)F)C(=O)O)c2ccc(F)cc2)cc1</smiles>
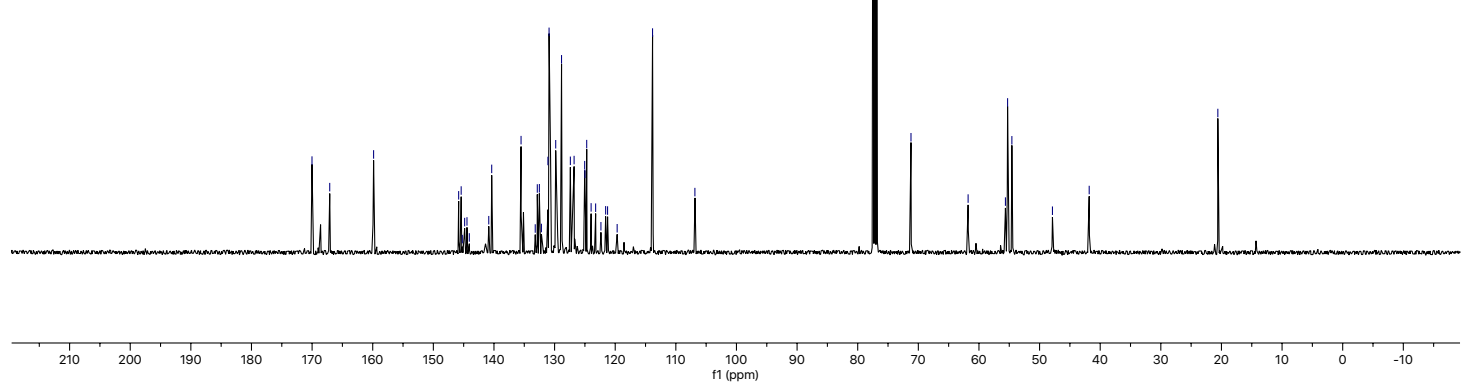


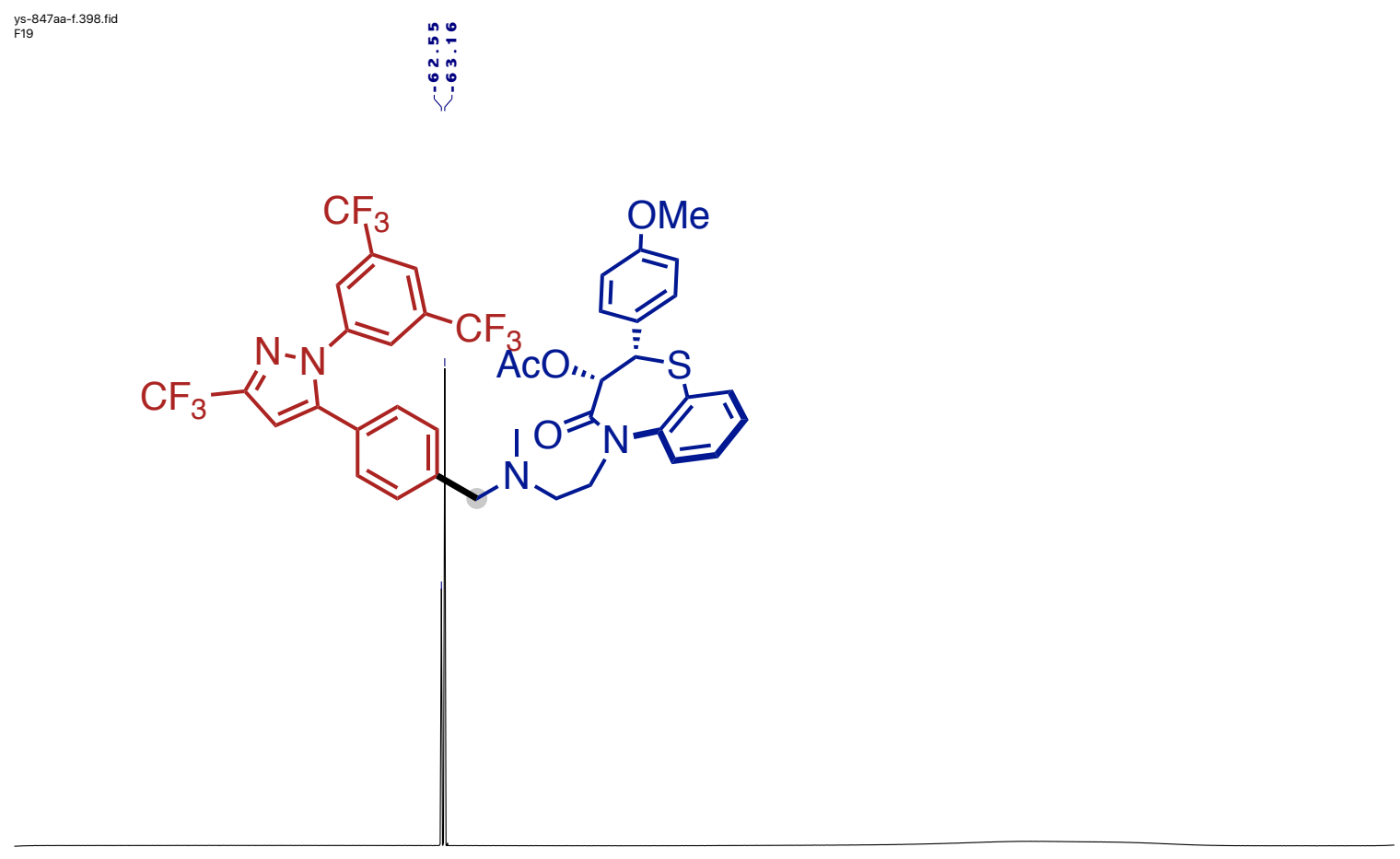

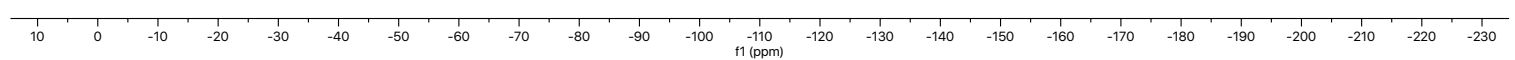




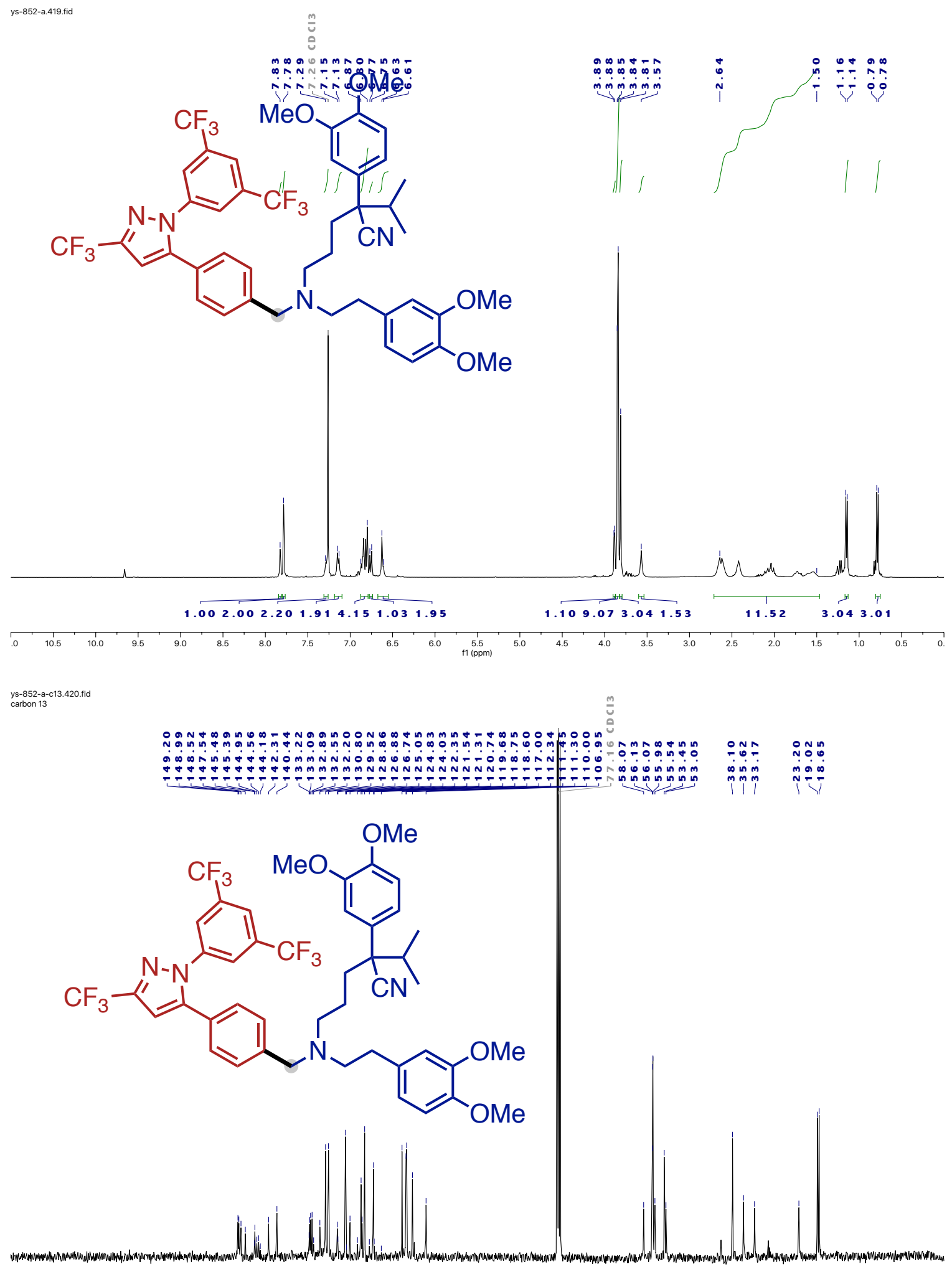

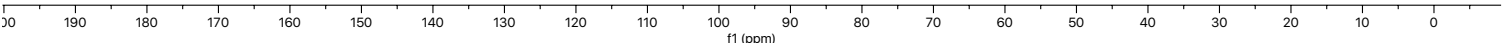



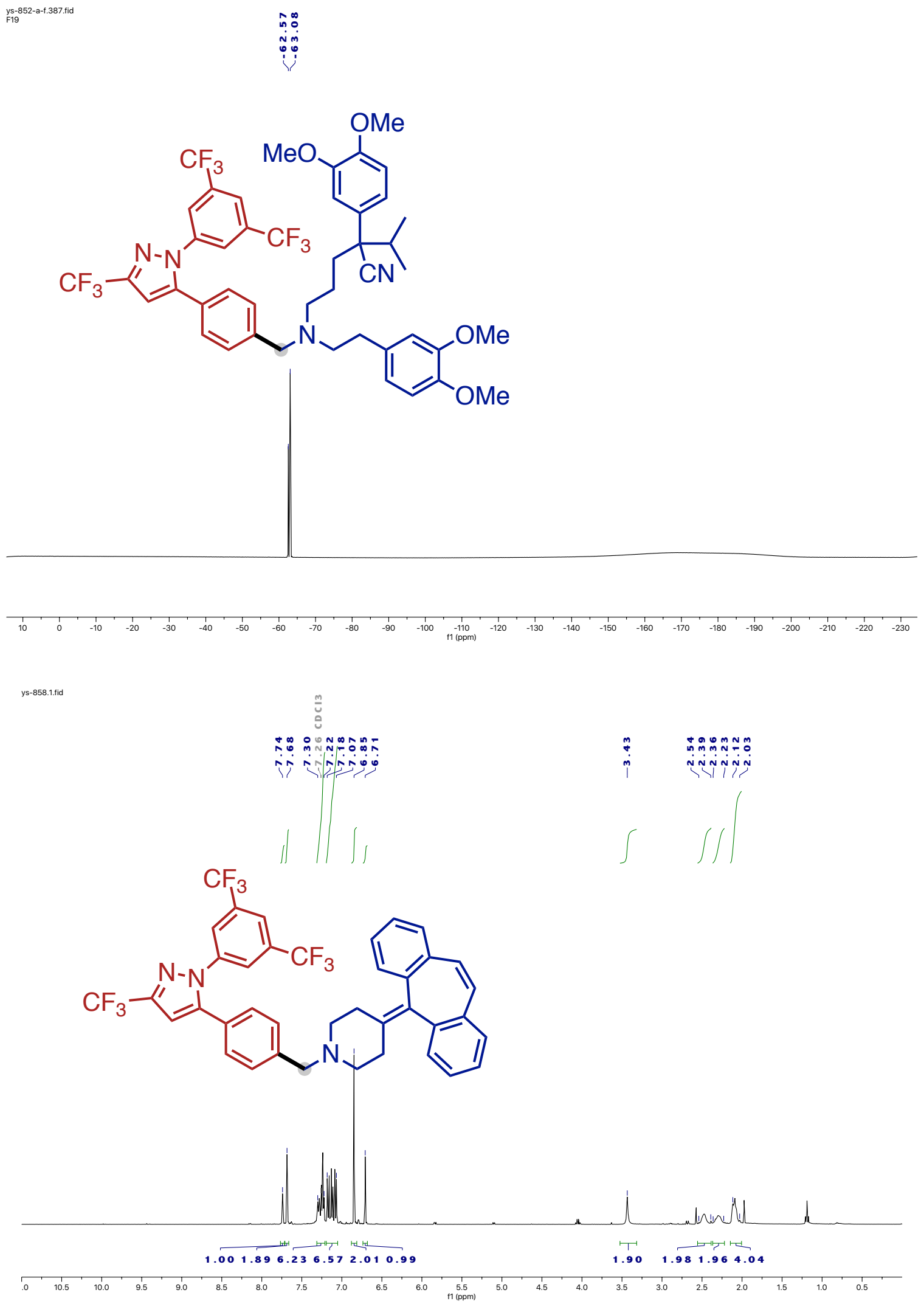

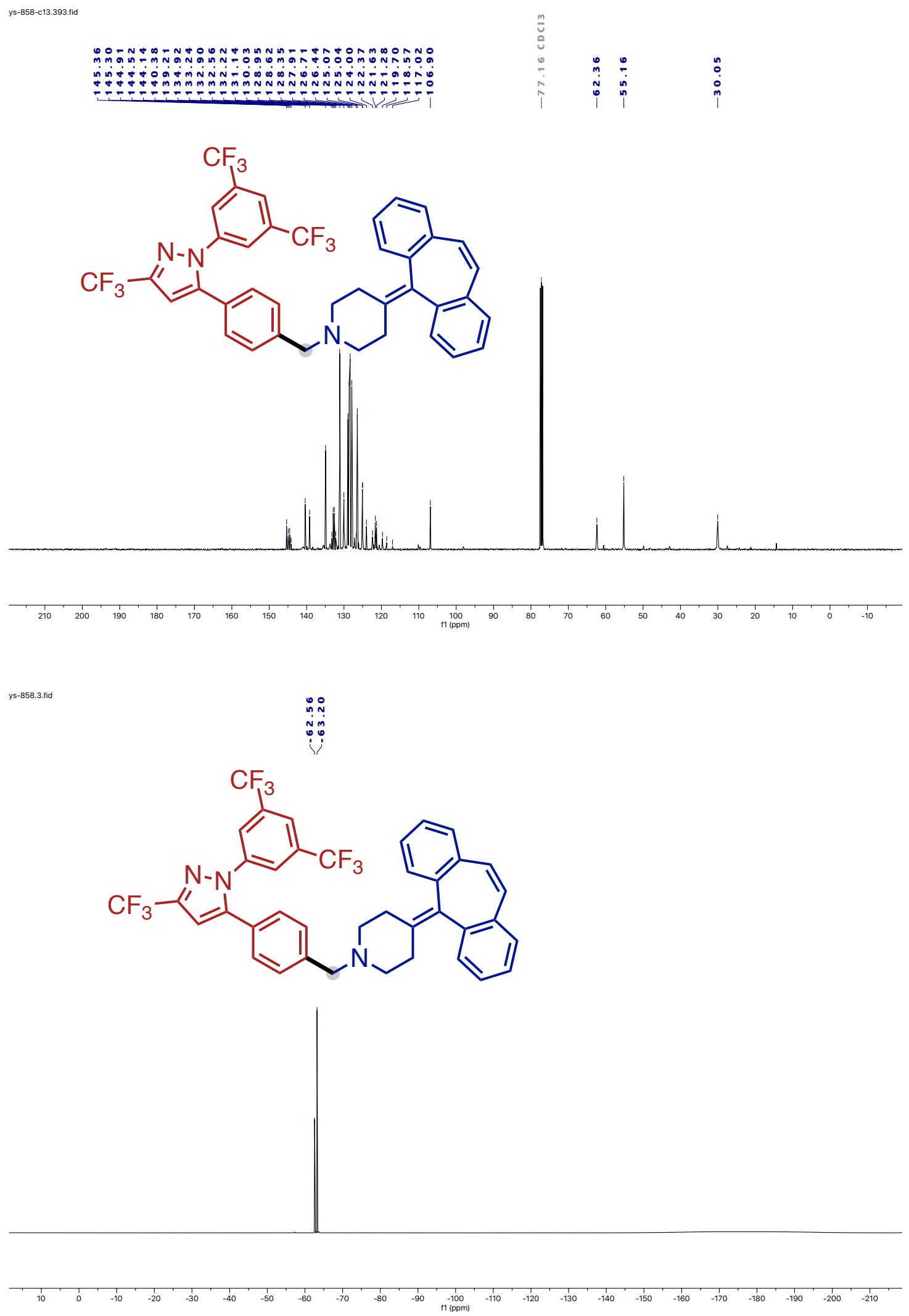

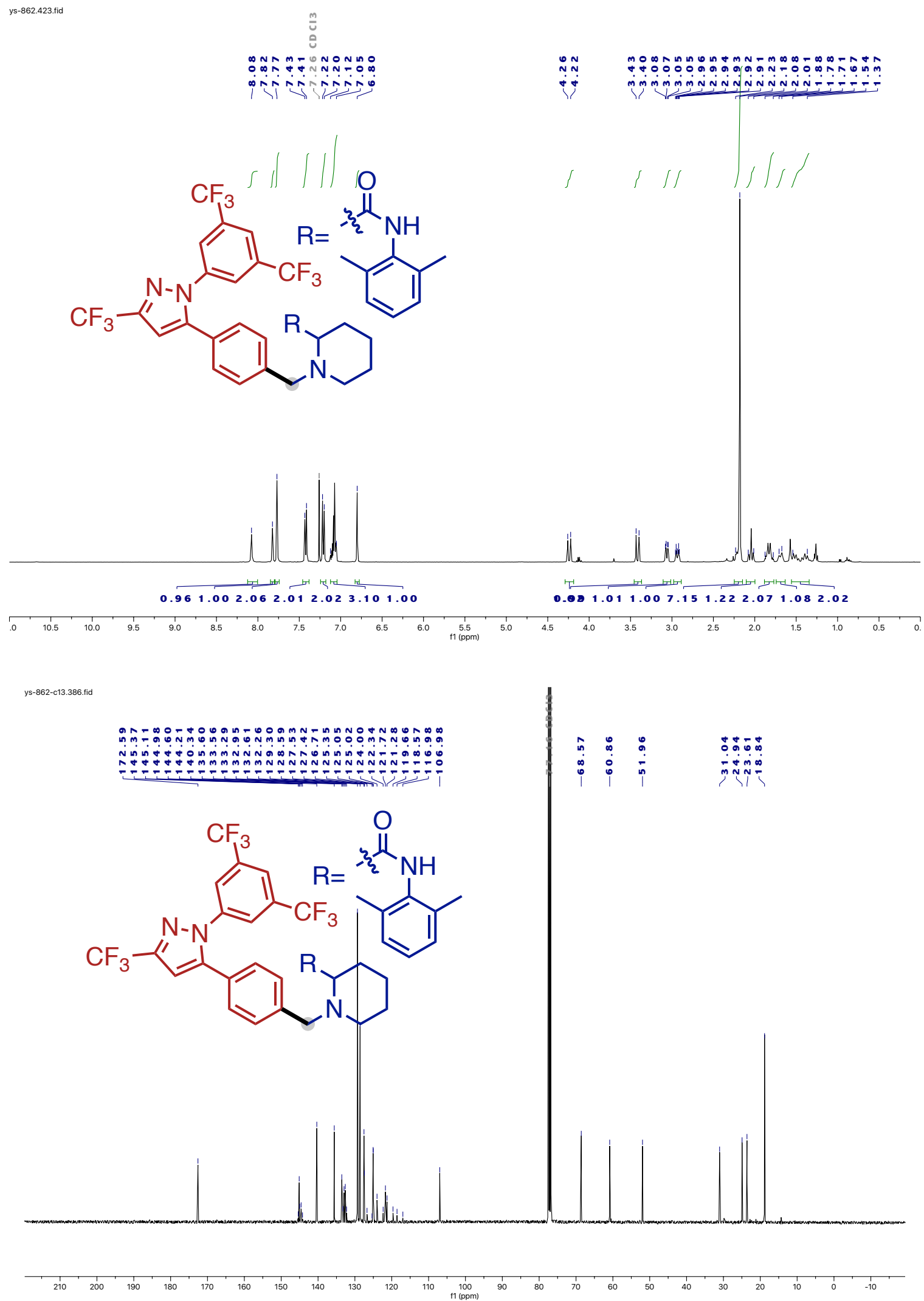

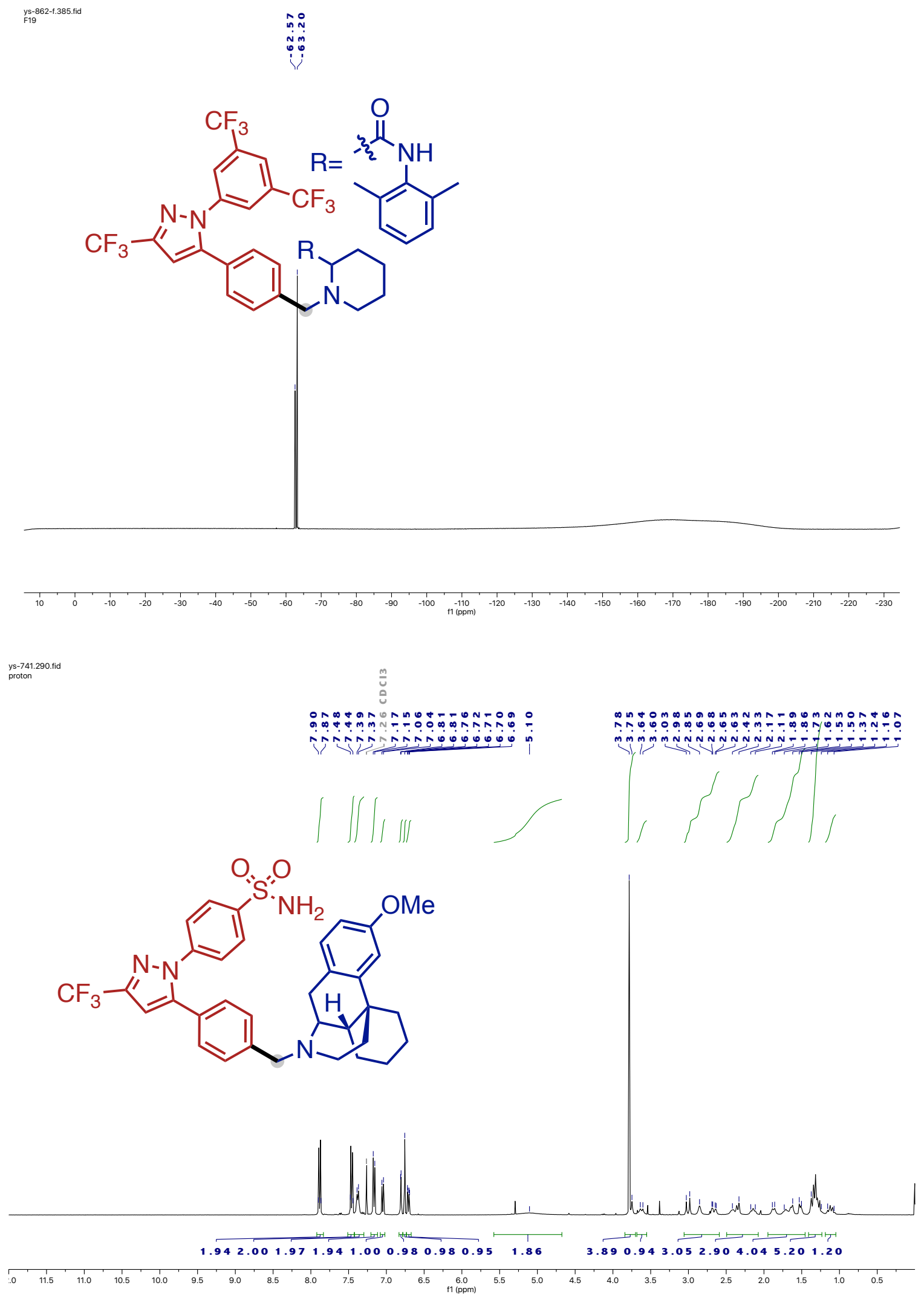

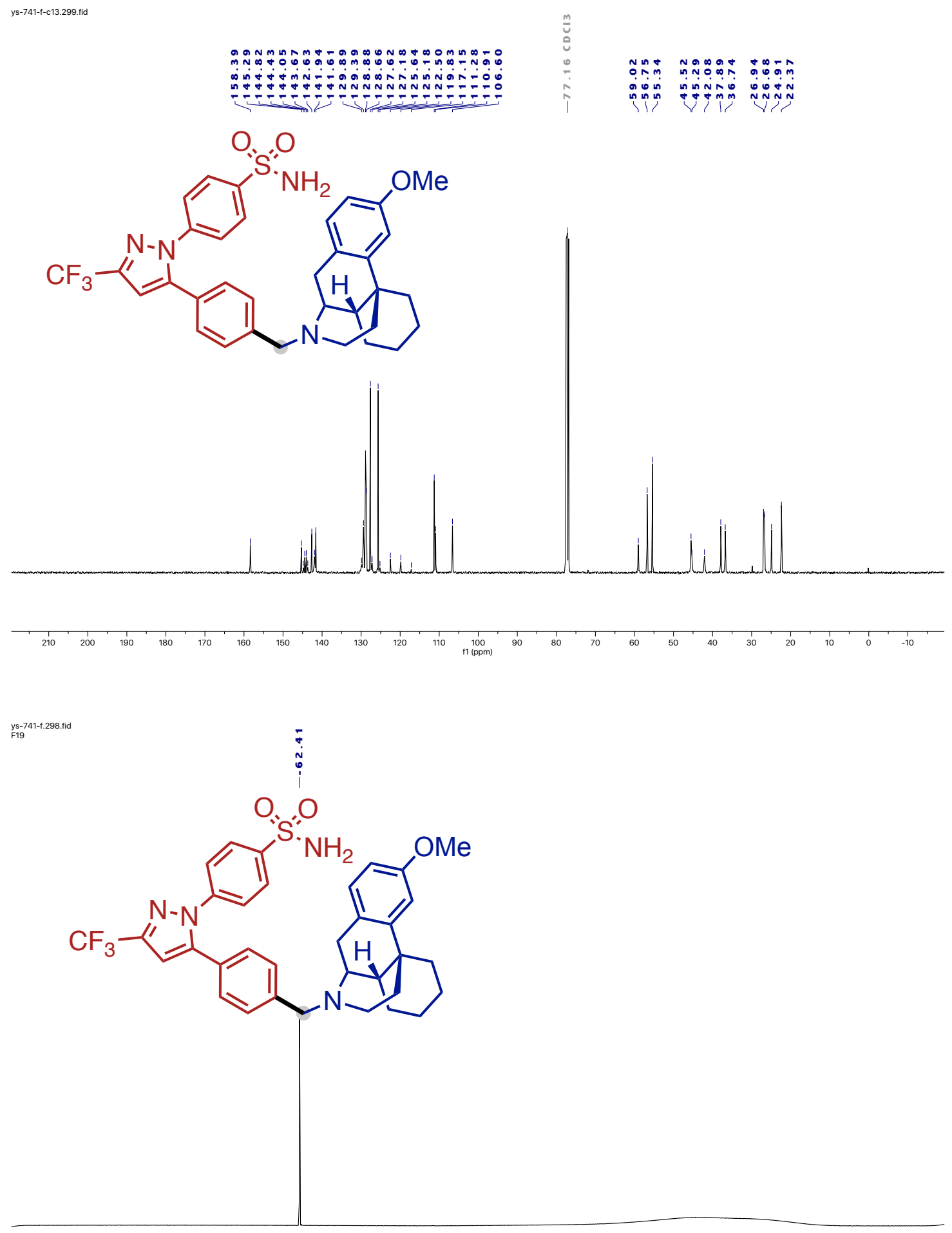